\title{
On the Extremum Properties of Thermodynamic Steady State in Non-Linear Systems
}

\author{
Gy. Vincze and A. Szasz \\ Department of Biotechnics, St.Istvan University, \\ Hungary
}

\section{Introduction}

The starting point of irreversible thermodynamics is the local form of the entropy balance equation. In a wide range of non-equilibrium processes, for instance heat conduction, thermo-diffusion non reactive mixture and thermoelectric effects are taking place in a volume $V$ in static conditions, the entropy balance has the following form

$$
\frac{\partial s}{\partial t}+\nabla \cdot \mathbf{j}_{s}=\sigma
$$

where

$$
\cdot \mathbf{j}_{s}=\sum_{i=1}^{N} \Gamma_{i} \mathbf{j}_{i} .
$$

is the entropy current density and

$$
\sigma=\sum_{i=1}^{N} \nabla \Gamma_{i} \cdot \mathbf{j}_{i}
$$

is the spontaneous internal entropy production, which is fixed non-negative by the second law of thermodynamics $\left.\sigma \geq 0\left[{ }^{1}\right],{ }^{2}\right]$. The symbols are: $\mathbf{j}_{i}$ is the current density vector of the i-th component, belonging to the extensive state variable $a_{i} ; \Gamma_{i}$ is the i-th intensive scalar variable and $\nabla \Gamma_{i}$ is the $\mathrm{i}$-th thermodynamic force (gradient of the intensive scalar $\Gamma_{i}$ ). The state variables $a_{i},(i=1,2, \ldots, N)$ and current densities $\mathbf{j}_{i},(i=1,2, \ldots, N)$ are assumed to obey local balance equations of the form

$$
\frac{\partial a_{i}}{\partial t}+\nabla \cdot \mathbf{j}_{i}=\sigma_{i},(i=1,2, \ldots, N)
$$

where $\sigma_{i}$ is the source of $a_{i}$.

Let us construct a more concise notation:

$$
\mathbf{j}:=\left(j_{1}=j_{11}, j_{2}=j_{12}, j_{3}=j_{13}, . ., j_{3 i-2}=j_{i 1}, . ., j_{3 N}=j_{N 3}\right)
$$


(and) 3N dimensional thermodynamic flux and the

$$
\mathbf{X}:=\left(X_{1}=\frac{\partial \Gamma_{1}}{\partial x_{1}}, X_{2}=\frac{\partial \Gamma_{1}}{\partial x_{2}}, X_{2}=\frac{\partial \Gamma_{1}}{\partial x_{3}}, . ., X_{3 i-2}=\frac{\partial \Gamma_{i}}{\partial x_{1}}, . ., X_{3 N}=\frac{\partial \Gamma_{N}}{\partial x_{3}}\right)
$$

conjugate thermodynamic force vector from the coordinates of the current densities $\mathbf{j}_{i}$ and $\nabla \Gamma_{i}$, respectively. The spontaneous entropy production (3) can then be written as a bilinear form

$$
\sigma=\sum_{i=1}^{N} \nabla \Gamma_{i} \cdot \mathbf{j}_{i}=\sum_{j=1}^{3 N} X_{j} j_{j}
$$

To make the notation simple, we drop out the summation symbol adopting the standard summation convention:

$$
\sigma=\sigma\left(X_{j}, j_{j}\right)=X_{j} j_{j}
$$

where any index repeated twice must be summed from 1 to $3 \mathrm{~N}$.

The $X^{\prime}$ s depend on the thermodynamic fluxes $j_{j}$. They can also depend on the intensive thermodynamic scalar variables $\Gamma_{k}$. We accordingly make the constitutive assumptions

$$
X_{j}=X_{j}\left(j_{l}, \Gamma_{k}\right),(j=1,2, \ldots, 3 N)
$$

Thus from (7) and (8) we get

$$
\sigma=X_{j} j_{j}=\sigma\left(j_{l} ; X_{j}\left(j_{l}, \Gamma_{k}\right)\right)=\sigma^{*}\left(j_{l}, \Gamma_{k}\right) \geq 0
$$

The thermodynamic fluxes and the entropy production vanish at thermodynamic equilibrium

$$
\left.\sigma^{*}\right|_{e q}=\sigma^{*}\left(0, \Gamma_{k}\right)=0
$$

and hence $j_{j}=0$ is a minimizing point of $\sigma^{*}\left(j_{l}, \Gamma_{k}\right)$, thus we have

$$
\left.\frac{\partial \sigma^{*}}{\partial j_{j}}\right|_{j_{l}=0}=0
$$

The differentiation of (10) and the above relations imply the equilibrium conditions

$$
\left.X_{j}\right|_{e q}=X_{j}\left(0, \Gamma_{k}\right)=0,(j=1,2, \ldots, 3 N)
$$

\subsection{The Onsager constitutive theory and its non-linear generalization}

The Onsager's irreversible thermodynamics [3] states linear constitutive relations of the form

$$
X_{j}=R_{j l}\left(\Gamma_{k}\right) j_{l}
$$


where the kinetic coefficients $R_{j l}\left(\Gamma_{k}\right)$ are independent of the $j$ 's and satisfy the well known symmetry, the reciprocity relations

$$
R_{j l}=R_{l j}
$$

The coefficients $R_{j l}\left(\Gamma_{k}\right)$ must satisfy the second law of thermodynamics too

$$
\sigma^{*}=X_{j}\left(j_{l}, \Gamma_{k}\right) j_{j}=j_{j} R_{j l} j_{l} \geq 0
$$

Due to the conditions (15) and (16) $R_{j l}$ is positive definite matrix. Furthermore, there are two independent requirements, for the constitutive relations (13): the reciprocity relations (15) and the second law of thermodynamics $\sigma^{*} \geq 0$. Additionally, the differentiation of (13) gives

$$
\frac{\partial X_{j}}{\partial j_{l}}=R_{j l}
$$

and hence the reciprocity relations (15) can be written in the equivalent form

$$
\frac{\partial X_{j}}{\partial j_{l}}=\frac{\partial X_{l}}{\partial j_{j}}
$$

This form of the reciprocity relations is also independent of the assumption that the constitutive relations are linear in the $j^{\prime}$ s. The equilibrium conditions (13) are always satisfied, independently of the actual form of the constitutive relations.

In the following we are going to introduce the generalized Onsager constitutive theory for a non-linear system of constitutive (9) satisfying the (18) reciprocity relations, the (13) equilibrium conditions and the (10) second law of thermodynamics. In the following we shall present that the Edelen's decomposition theorem [ [ ${ }^{4}$ is valid in every class of the thermodynamic forces, which are two times continuously differentiable with respect to fluxes.

This theorem states that every thermodynamic force can be decomposed into the sum of two unique forces. The first identically satisfies the reciprocity relations and the second gives an identically vanished entropy production. The first type of forces can be a deduction of a so called flux dissipation potential.

Let us denote the thermodynamic forces of any system in general by $X_{j}=X_{j}\left(j_{l}, \Gamma_{k}\right)$. There are existing unique forces $X_{j}^{*}\left(j_{l}, \Gamma_{k}\right)$ and $V_{j}\left(j_{l}, \Gamma_{k}\right)$ for each $X_{j}\left(j_{l}, \Gamma_{k}\right)$ like

$$
\begin{aligned}
& X_{j}\left(j_{l}, \Gamma_{k}\right)=X_{j}^{*}\left(j_{l}, \Gamma_{k}\right)+V_{j}\left(j_{l}, \Gamma_{k}\right), \\
& \frac{\partial X_{j}^{*}}{\partial j_{l}}=\frac{\partial X_{l}^{*}}{\partial j_{j}},
\end{aligned}
$$

and

$$
j_{j} V_{j}\left(j_{l}, \Gamma_{k}\right)=0
$$


which is identically satisfied with every variable $\left(j_{l}, \Gamma_{k}\right)$, where

$$
\begin{aligned}
& X_{j}^{*}\left(j_{l}, \Gamma_{k}\right)=\frac{\partial \Phi\left(j_{l}, \Gamma_{k}\right)}{\partial j_{j}}, \\
& \Phi\left(j_{l}, \Gamma_{k}\right)=\int_{0}^{1} j_{j} X_{j}\left(\tau j_{l}, \Gamma_{k}\right) d \tau \\
& V_{j}\left(j_{l}, \Gamma_{k}\right)=\int_{0}^{1} j_{m}\left(\frac{\partial X_{j}\left(\tau j_{l}, \Gamma_{k}\right)}{\partial \tau j_{m}}-\frac{\partial X_{m}\left(\tau j_{l}, \Gamma_{k}\right)}{\partial \tau j_{j}}\right) d \tau
\end{aligned}
$$

This is the Edelen's decomposition theorem. Its essence is that every force has a definite separate part $X_{j}^{*}\left(j_{l}, \Gamma_{k}\right)$ which satisfies the reciprocity relations (19) and a certain other separate part $V_{j}\left(j_{l}, \Gamma_{k}\right)$, which identically satisfies the relation (20). We refer to this second type of force as non dissipative force. The flux dissipation potential $\Phi\left(j_{l}, \Gamma_{k}\right)$ is uniquely determined to within an additive function of $\Gamma^{\prime}$ s. A direct consequence of Edelen 's theorem is when the thermodynamic forces are Onsager forces and this assertion is equivalent to the assumption of the existence of physical systems with zero non dissipative forces.

\subsection{The second law of thermodynamics and the dissipation potentials}

From the Edelen's decomposition theorem follows that the existence of the scalar potential $\Phi\left(j_{l}, \Gamma_{k}\right)$ and the associated thermodynamic forces

$$
X_{j}\left(j_{l}, \Gamma_{k}\right)=\frac{\partial \Phi\left(j_{l}, \Gamma_{k}\right)}{\partial j_{j}}
$$

which satisfy the reciprocity relation, and these also have to have to satisfy all the supplementary conditions of the generalized Onsager constitutive theory. A/One consequence of the second law of thermodynamics is the existence of a so called flux dissipation potential $\Phi\left(j_{l}, \Gamma_{k}\right)$ which satisfies the inequality (10), in every case, i.e.

$$
\sigma^{*}\left(j_{l}, \Gamma_{k}\right)=\sigma\left(j_{l} ; X_{j}\left(j_{l}, \Gamma_{k}\right)\right)=j_{j} \frac{\partial \Phi\left(j_{l}, \Gamma_{k}\right)}{\partial j_{j}} \geq 0 .
$$

From the equations (11) and (12) follows

$$
\left.\frac{\partial \Phi\left(j_{l}, \Gamma_{k}\right)}{\partial j_{j}}\right|_{j_{i}=0}=X_{j}\left(j_{l}=0, \Gamma_{k}\right)=0 .
$$

There are two further important properties of the thermodynamic flux potential $\Phi\left(j_{l}, \Gamma_{k}\right)$, [4], namely

$$
\begin{aligned}
& \Phi\left(j_{l}, \Gamma_{k}\right)>0 \quad \text { for } j_{l} \neq 0, \\
& \Phi\left(0, \Gamma_{k}\right)=0
\end{aligned}
$$


Also, the potential $\Phi\left(j_{l}, \Gamma_{k}\right)$ is strictly positive with respect to $j$ 's, except in equilibrium point $j_{l}=0,(l=1,2, \ldots, 3 N)$. The above three relations however, do not guarantee that the dissipation-potential $\Phi\left(j_{l}, \Gamma_{k}\right)$ is a convex function with respect to the thermodynamic fluxes.

Introducing the entropy production into the second equation of (21) like

$$
\begin{aligned}
& \Phi\left(j_{l}, \Gamma_{k}\right)=\int_{0}^{1} \tau j_{j} X_{j}\left(\tau j_{l}, \Gamma_{k}\right) \frac{d \tau}{\tau}=\int_{0}^{1} \sigma^{*}\left(\tau j_{j}, \Gamma_{k}\right) \frac{d \tau}{\tau}= \\
& =\int_{0}^{\infty} \sigma^{*}\left(e^{-t} j_{j}, \Gamma_{k}\right) d t
\end{aligned}
$$

where we change the integration variable by the substitution $\tau=e^{-t}$. Also, the flux dissipation potential in the $\left(j_{l}, \Gamma_{k}\right)$ state is equal to the time integral of the entropy production in the pure relaxation processes $j_{j} e^{-t}$ from the initial states $j_{j}$ to the equilibrium state. This is the physical meaning of the dissipation potential.

\subsection{On the validity of Onsager's reciprocal relations}

The original Onsager's proof of reciprocal relation is based on three assumptions and on the principle of microscopic reversibility. The first is that the constitutive equations are linear. The second is the so called regression hypothesis. The third Onsager's assumption is that the thermodynamic fluxes are time derivatives of state variables [3]. However, in the case of vector flows, this condition is not satisfied. For example the heat flux vector and the viscous pressure tensor cannot be expressed in such simple time-derivative form in classical nonequilibrium thermodynamics. On the other hand, as Casimir pointed out [5]: the proof of this reciprocity relations is based directly on the hypothesis that the regression of fluctuations follows the same laws as macroscopic irreversible processes do. This is the regression hypothesis. Consequently, the use of the Onsager's reciprocal relation describing of macroscopic phenomena must be based on experimental verification or we have to accept Truesdell's opinion [6]: „if the reciprocal relations are correct we have to derive them by pure phenomenology also".

The experimental investigations prove the validity of the reciprocal relations for several types of irreversible processes; moreover, as we show below the linear and generalized reciprocal relations are clear phenomenological consequence of the principle of minimal entropy production. Consequently, the generalized reciprocal relations are reasonably wellestablished thermodynamic law, however of course it has no such general validity as the basic laws of thermodynamics, (e.g. the energy conservation or the direction of the spontaneous thermodynamic processes).

Berdichevsky [7] showed in the case of non-linear constitutive equations, when the macro parameters of the thermodynamic system as stochastic variables are Markov-type ones and satisfy the Focker-Planck equation, as well as stationer and stochastically reversible in meaning of Kolmogorov's conditions, then the non-linear constitutive equations of the thermodynamic fluxes and forces fulfill the generalized reciprocal relations. It is specially interesting, that Berdichevsky's results are based on the necessary and sufficient conditions of reversibility of Markov-processes obtained by Kolmogorov in 1937 [8]. Kolmogorov had formulated his reversibility relation on $\alpha$-type macro-variables which do not change their sign by reverse process. The necessary and sufficient conditions of reversibility for stochastic 
processes on $\beta$-type variables (such variables which change their sign in reverse process) were formulated by Yaglom [ $\left.{ }^{9}\right]$.

The Markov-character of macro-variables was observed in various physical systems [10], consequently, the generalized Onsager's reciprocal relations are expected to be correct in numerous physical systems. This is supported by another result of Berdichevsky, [11], showing that the thermodynamically slow macro variables are parts of these equations when the dynamics of the system is governed by Hamiltonian equations. When we integrate the Liuville-equation of the above system on the rapidly changing variables, the probability density function of the thermodynamic distribution of the slow changing stochastic macro variables satisfy the Focker-Planck equation. Combining this result with the flux-hypothesis on the probability of current densities, the generalized Onsager's reciprocal relation could be obtained. Consequently, the Hamiltonian character of the macro-dynamics together with some further plausible conditions verify the reciprocal relations. This generalized reciprocal relation may be considered as a reasonably well-established law, but less rigorously valid than the Onsager's original one.

\subsection{Minimum principle of the generalized Onsager constitutive theory}

Limit our investigation on the generalized Onsager's constitutive theory, containing the linear Onsager's theory as well. In this case the thermodynamic fluxes have no non dissipative parts and the thermodynamic forces could be deduced from the flux dissipation potential by the Edelens' decomposition theorem. Assuming a dissipation potential connecting the thermodynamic forces and fluxes like:

$$
X_{i}=\frac{\partial \Phi\left(J_{k}, \Gamma_{l}\right)}{\partial J_{i}},(i=1,2, \ldots, 3 N)
$$

In the following part our aim is to introduce the Legendre transform of flux potential $\Phi\left(J_{k}, \Gamma_{l}\right)$.

To perform a Legendre transform, it is necessary that the above constitutive equations are invertible to the fluxes. It is possible, when from the derivate functions $\frac{\partial \Phi\left(J_{k}, \Gamma_{l}\right)}{\partial J_{i}}$ as coordinates constructed

$$
\mathbf{X}=\mathbf{X}\left(\mathbf{J}, \Gamma_{l}\right):=\nabla_{\mathbf{J}} \Phi\left(\mathbf{J}, \Gamma_{l}\right)
$$

gradient mapping is a one-to-one and onto (transformation) mapping between the two open sets of fluxes and forces for any $\frac{\partial \Phi\left(J_{k}, \Gamma_{l}\right)}{\partial J_{i}}$ 's, where $\mathbf{J}=\left(J_{1}, J_{2}, \ldots, J_{3 N}\right)$ and $\mathbf{X}=\left(X_{1}, X_{2}, \ldots, X_{3 N}\right)$ are vectors of $3 N$ dimensions obtained from the fluxes and forces. To give a criteria to the invertibility of (27) we introduce the so called Hessian matrix [12]:

$$
\mathbf{H} \Phi\left(\mathbf{J}, \Gamma_{l}\right)=\left[\frac{\partial^{2} \Phi\left(\mathbf{J}, \Gamma_{l}\right)}{\partial J_{i} \partial J_{i}}\right]
$$

Let $G$ be a convex open set and $C^{2}$ be the space of all two times continuously differentiable functions. 
The flux potential is strictly convex by definition, when $\Phi \in C^{2}(G)$ and the Hessian matrix of $\Phi$ is positive definite on $G\left[{ }^{13}\right],\left[{ }^{14}\right]$, i.e.

$$
\left[\mathbf{H} \Phi\left(\mathbf{J}, \Gamma_{l}\right) \mathbf{J}\right] \cdot \mathbf{J}=\frac{\partial^{2} \Phi\left(\mathbf{J}, \Gamma_{l}\right)}{\partial J_{k} \partial J_{i}} J_{i} J_{k}>0, \forall \mathbf{J} \neq 0
$$

When the flux potential is strictly convex, then the gradient mapping (28) is one-to-one and onto. This condition is sufficient to perform the Legendre transform as follows:

The partial Legendre transform of $\Phi\left(\mathbf{J}, \Gamma_{l}\right)$ is defined by solving $\mathbf{X}=\mathbf{X}\left(\mathbf{J}, \Gamma_{l}\right)$ for $\mathbf{J}=\mathbf{J}\left(\mathbf{X}, \Gamma_{l}\right)$, and then setting

$$
\begin{aligned}
& \Psi\left(\mathbf{X}, \Gamma_{l}\right)=\mathbf{X} \cdot \mathbf{J}-\Phi\left(\mathbf{J}, \Gamma_{l}\right)= \\
& =\mathbf{X} \cdot \mathbf{J}\left(\mathbf{X}, \Gamma_{l}\right)-\Phi\left(\mathbf{J}\left(\mathbf{X}, \Gamma_{l}\right), \Gamma_{l}\right)
\end{aligned}
$$

In the theory of Legendre transformation we refer to the fluxes $\mathbf{J}=\left(J_{1}, J_{2}, \ldots, J_{3 N}\right)$ as original coordinates, while the forces $\mathbf{X}=\left(X_{1}, X_{2}, \ldots, X_{3 N}\right)$ will be the conjugate coordinates. The symbol $\mathbf{X} \cdot \mathbf{J}$ is the dot product, so

$$
\Psi\left(\mathbf{X}, \Gamma_{l}\right)=\mathbf{X} \cdot \mathbf{J}-\Phi\left(\mathbf{J}, \Gamma_{l}\right)=\Psi\left(X_{j}, \Gamma_{l}\right)=X_{i} \cdot J_{i}-\Phi\left(J_{j}, \Gamma_{l}\right)
$$

Proposition. The force potential $\Psi\left(X_{j}, \Gamma_{l}\right)$ could be derived two times continuously by forces, and

$$
\nabla_{\mathbf{X}} \Psi\left(\mathbf{X}, \Gamma_{l}\right)=\mathbf{J}\left(\mathbf{X}, \Gamma_{l}\right)=\mathbf{J}, \nabla_{\Gamma} \Phi\left(\mathbf{J}, \Gamma_{l}\right)=-\nabla_{\Gamma} \Psi\left(X_{j}, \Gamma_{l}\right), \Gamma=\left(\Gamma_{l}, \Gamma_{l}, \ldots, \Gamma_{l}\right)
$$

By calculating differential of the force potential

$$
\begin{aligned}
& d \Psi\left(\mathbf{X}, \Gamma_{l}\right)=\nabla_{\mathbf{X}} \Psi\left(\mathbf{X}, \Gamma_{l}\right) \cdot d \mathbf{X}+\nabla_{\boldsymbol{\Gamma}} \Psi\left(\mathbf{X}, \Gamma_{l}\right) \cdot d \boldsymbol{\Gamma}= \\
& =d \mathbf{X} \cdot \mathbf{J}+\mathbf{X} \cdot d \mathbf{J}-\nabla_{\mathbf{J}} \Phi\left(\mathbf{J}, \Gamma_{l}\right) \cdot d \mathbf{J}-\nabla_{\mathbf{\Gamma}} \Phi\left(\mathbf{J}, \Gamma_{l}\right) \cdot d \boldsymbol{\Gamma}= \\
& =d \mathbf{X} \cdot \mathbf{J}-\nabla_{\boldsymbol{\Gamma}} \Phi\left(\mathbf{J}, \Gamma_{l}\right) \cdot d \boldsymbol{\Gamma}
\end{aligned}
$$

since $\nabla_{\mathbf{J}} \Phi\left(\mathbf{J}, \Gamma_{l}\right)=\mathbf{X}$. Hence

$$
\begin{gathered}
\nabla_{\mathbf{X}} \Psi\left(\mathbf{X}, \Gamma_{l}\right)=\mathbf{J}=\mathbf{J}\left(\mathbf{X}, \Gamma_{l}\right), \\
\nabla_{\mathbf{\Gamma}}\left[\Phi\left(\mathbf{J}, \Gamma_{l}\right)+\Psi\left(X, \Gamma_{l}\right)\right]=0
\end{gathered}
$$

The equation (36) is referred to further as Donkin's theorem [14].

It can be shown that the Hessian matrix of $\Psi\left(X_{j}, \Gamma_{l}\right)$ is positive definite, so if the range of gradient mapping (28) is a convex open set, then the force dissipation potential is also strictly convex.

Since the force dissipation potential $\Psi\left(X_{j}, \Gamma_{l}\right)$ has continuous second derivative with respect to forces it follows that the generalized reciprocal relations:

$$
\frac{\partial \Psi^{2}\left(X_{j}, \Gamma_{l}\right)}{\partial X_{k} \partial X_{i}}=\frac{\partial \Psi^{2}\left(X_{j}, \Gamma_{l}\right)}{\partial X_{i} \partial X_{k}},(i, k=1,2, \ldots, 3 N)
$$


The Legrende transform by these above assumptions is an involution, which means applying the transformation on the force dissipation potential, the result will be the flux potential.

\subsubsection{The Onsager-Machlup-function}

The Legendre transformation could be formulated as an extremum-task as well [15]. The Onsager-Machlup function (OM-function) play a central role in the extremum principles of the irreversible thermodynamics based on generalized Onsager constitutive theory. The OM-function could be introduced by the spontaneous entropy production and one of dissipation potentials using the Legendre transformation.

Let the open convex sets $G$ and $G^{*}$ be the domain of $\Phi\left(\mathrm{J}, \Gamma_{l}\right)$ and range of the gradient mapping (28) respectively. If $\Phi\left(\mathrm{J}, \Gamma_{l}\right)$ is strictly convex then

$$
\Psi\left(\mathbf{X}, \Gamma_{l}\right)=\max _{\mathbf{J} \in G}\left[\mathbf{X} \cdot \mathbf{J}-\Phi\left(\mathbf{J}, \Gamma_{l}\right)\right], \forall \mathbf{X} \in G^{*}
$$

Indeed, the necessary condition of the extremum of $\Psi\left(\mathbf{X}, \Gamma_{l}\right)$ is $\mathbf{X}\left(\mathbf{J}, \Gamma_{l}\right)=\nabla_{\mathbf{J}} \Phi\left(\mathbf{J}, \Gamma_{l}\right)$. Its solution for $\mathbf{J}$ substituted into (38), we obtain the definition (31) of Legendre transform. Due to the $\mathbf{X} \cdot \mathbf{J}-\Phi\left(\mathbf{J}, \Gamma_{l}\right)$ can only be equal with its maximum, thus in case of strictly convex $\Phi\left(\mathbf{J}, \Gamma_{l}\right)$ and $\Psi\left(\mathbf{X}, \Gamma_{l}\right)$ dissipation potentials we get the Young-inequality from (38):

$$
\mathbf{X} \cdot \mathbf{J} \leq \Psi\left(\mathbf{X}, \Gamma_{l}\right)-\Phi\left(\mathbf{J}, \Gamma_{l}\right), \forall \mathbf{X} \in G^{*}, \forall \mathbf{J} \in G
$$

where the equality can be satisfied only when the constitutive equations (28) and (35) are satisfied. Defining the Onsager-Machlup function as:

$$
\begin{aligned}
& o\left(\mathbf{X}, \mathbf{J}, \Gamma_{l}\right)=\Psi\left(\mathbf{X}, \Gamma_{l}\right)+\Phi\left(\mathbf{J}, \Gamma_{l}\right)-\mathbf{X} \cdot \mathbf{J}= \\
& =\Psi\left(\mathbf{X}, \Gamma_{l}\right)+\Phi\left(\mathbf{J}, \Gamma_{l}\right)-\sigma(\mathbf{X}, \mathbf{J}) \geq 0,
\end{aligned}
$$

Also, this contains the entropy production, and was introduced first by Onsager and Machlup in their work about non-equilibrium fluctuation theory [16]. With this we can formulate the minimum theorem of the generalized Onsager constitutive theory: the OMfunction is the non-negative function of the fluxes, forces and intensive parameters. It only becomes zero, which is its minimum, when the material equations of the generalized Onsager constitutive theory are satisfied. The OM-function has crucial importance, because it contains all the important constitutive properties of the linear and generalized constitutive theories; these follow from the necessary conditions of the minimum of OM-function:

$$
\begin{aligned}
& \nabla_{\mathbf{\Gamma}} o(\mathbf{X}, \mathbf{J})=0 \rightarrow \nabla_{\Gamma}\left[\Phi\left(\mathbf{J}, \Gamma_{l}\right)+\nabla_{\mathbf{X}} \Psi\left(\mathbf{X}, \Gamma_{l}\right)\right]=0, \\
& \nabla_{\mathbf{J}} o(\mathbf{X}, \mathbf{J})=0 \rightarrow \nabla_{\mathbf{J}} \Phi\left(\mathbf{J}, \Gamma_{l}\right)=\mathbf{X} \\
& \nabla_{\mathbf{X}} o(\mathbf{X}, \mathbf{J})=0 \rightarrow \nabla_{\mathbf{X}} \Psi\left(\mathbf{X}, \Gamma_{l}\right)=\mathbf{J}
\end{aligned}
$$

With little exaggeration we can state the OM-function has similar role in the constitutive theory of the non-equilibrium thermodynamics to the Hamiltonian in the dynamics of reversible processes. An important consequence of the Donkin's theorem is the gradient of 
the spontaneous entropy production by the intensive parameters is zero in the generalized Onsager constitutive theory:

$$
\nabla_{\mathbf{\Gamma}} \sigma(\mathbf{X}, \mathbf{J})=0 \text {, if } \nabla_{\mathbf{J}} \Phi\left(\mathbf{J}, \Gamma_{l}\right)=\mathbf{X}, \nabla_{\mathbf{X}} \Psi\left(\mathbf{X}, \Gamma_{l}\right)=\mathbf{J},
$$

We refer to this result as Donkin's theorem of the entropy production.

As we shall see later some dissipation potentials are homogeneous Euler's functions. This means for example, that the $\Phi\left(\mathrm{J}, \Gamma_{l}\right)$ is a homogeneous Euler's function of degree $k$ when every scalar of $\lambda \in R$ the following relation is valid:

$$
\Phi\left(\lambda \mathbf{J}, \Gamma_{l}\right)=\lambda^{k} \Phi\left(\mathbf{J}, \Gamma_{l}\right), \forall \mathbf{J} \in G
$$

It can be proved [17], that the dissipation potential $\Psi\left(\mathbf{X}, \Gamma_{l}\right)$ dual-pair of $\Phi\left(\mathbf{J}, \Gamma_{l}\right)$ is also homogeneous Euler's-function, like

$$
\Psi\left(\lambda \mathbf{X}, \Gamma_{l}\right)=\lambda^{m} \Psi\left(\mathbf{X}, \Gamma_{l}\right), \frac{1}{k}+\frac{1}{m}=1
$$

Substituting the (14) quasi-linear Onsager constitutive equations for the second equation of the Edelen's decomposition theorem (21), then we get for the flux potential

$$
\begin{aligned}
& \Phi\left(j_{l}, \Gamma_{k}\right)=\int_{0}^{1} j_{j} X_{j}\left(\tau j_{l}, \Gamma_{k}\right) d \tau=\int_{0}^{1} R_{j l}\left(\Gamma_{k}\right) j_{j} \tau j_{l} d \tau= \\
& =R_{j l}\left(\Gamma_{k}\right) j_{j} j_{l}\left[\frac{\tau^{2}}{2}\right]_{0}^{1}=\frac{1}{2} R_{j l}\left(\Gamma_{k}\right) j_{j} j
\end{aligned}
$$

Hence, based on the definition of (43), we see that the dissipation potentials are $2^{\text {nd }}$-degree homogeneous Euler's functions in Onsager's linear constitutive theory:

$$
\Phi\left(\lambda j_{l}, \Gamma_{k}\right)=\frac{1}{2} R_{j l}\left(\Gamma_{k}\right) \lambda j_{j} \lambda j=\lambda^{2} \frac{1}{2} R_{j l}\left(\Gamma_{k}\right) j_{j} j=\lambda^{2} \Phi\left(j_{l}, \Gamma_{k}\right)
$$

we can see from the definition (43) and the equation (44), that the dissipation potentials are homogeneous Euler's functions of degree two in the quasi-linear Onsager constitutive theory.

Consequently, a homogeneous Euler's function of degree two dissipation potential belongs to the homogeneous Euler's function of degree one constitutive equations. This could be generalized to the homogeneous dissipative functions of any degrees: the constitutive equations describe one degree less homogeneous functions of fluxes and forces than the dissipation potentials associated to them.

\subsection{Example}

\subsubsection{Thermodynamic constrains. Supplementary thermodynamic forces}

Numerous differential theorems are well known in the classical mechanics of mass-points, like the theorem of virtual work, the Gauss-theorem, the D' Alambert theorem, etc. These are supporting the construction of the equations of motion and are especially useful to handle 
the constrained problems. The minimum theorem of constitutive theory, formulated by OMfunction, can be used similarly as above theorem in the non-linear constitutive theory of non-equilibrium thermodynamics.

As an example we study the transport processes of a membrane (e.g. cellular membranes) in the frame of Onsager constitutive theory. For simplicity we study the transport of only three chemical components (for cell membrane: $\mathrm{Na}, \mathrm{K}, \mathrm{Cl}$ ) and assume that the $T$ temperature is constant on the membrane. These transports are governed by the corresponding gradients of $\mu_{1}, \mu_{2}, \mu_{3}$ chemical potentials. Due to the chemical components are ions, with their transport not only the chemical species but electric charges are transported. Assuming that the net current density in every internal point of the membrane is zero. This is a local constraint. When the molar current density of chemical components are $\mathbf{j}_{1}, \mathbf{j}_{2}, \mathbf{j}_{3}$, the thermodynamic forces conjugated to these current densities are $\mathbf{X}_{1}:=-\nabla \frac{\mu_{1}}{T}, \quad \mathbf{X}_{2}:=-\nabla \frac{\mu_{2}}{T}$, $\mathbf{X}_{3}:=-\nabla \frac{\mu_{3}}{T}$, then the constrain can be formulated by the equation

$$
z_{1} \mathbf{j}_{1}+z_{2} \mathbf{j}_{2}+z_{3} \mathbf{j}_{3}=0
$$

where $z_{i}$ are the charges on the ions. The force potential knowing that the $\Phi\left(\mathbf{J}, \Gamma_{l}\right)$ flux potential can be deduced from the extremum-task determination based on equation (38), which has constraint of (47), i.e.

$$
\begin{aligned}
& \Psi\left(\mathbf{X}, \Gamma_{l}\right)=\max _{\mathbf{J} \in G}\left[\mathbf{X}_{i} \cdot \mathbf{J}_{i}-\Phi\left(\mathbf{J}_{i}, \Gamma_{l}\right)\right], \forall \mathbf{X} \in G^{*}, \\
& z_{1} \mathbf{j}_{1}+z_{2} \mathbf{j}_{2}+z_{C 3} \mathbf{j}_{3}=0
\end{aligned}
$$

We solve this constrained extremum problem by Lagrange's method of multipliers using the

$$
\begin{aligned}
& \max _{\mathbf{J} \in G} F\left(\mathbf{X}_{i}, \mathbf{J}_{i}, \boldsymbol{\lambda}, \Gamma_{l}\right) \\
& F\left(\mathbf{X}_{i}, \mathbf{J}, \boldsymbol{\lambda}, \Gamma_{l}\right):=\mathbf{X}_{i} \cdot \mathbf{J}_{i}-\Phi\left(\mathbf{J}_{i}, \Gamma_{l}\right)+\boldsymbol{\lambda} \cdot\left(z_{1} \mathbf{j}_{1}+z_{2} \mathbf{j}_{2}+z_{3} \mathbf{j}_{3}\right)
\end{aligned}
$$

free task. In this case the necessary conditions of the minima are:

$$
\nabla_{\mathbf{J}_{\mathbf{i}}} F\left(\mathbf{X}_{i}, \mathbf{J}_{i}, \lambda, \Gamma_{l}\right)=0 \rightarrow \nabla_{\mathbf{J}_{\mathbf{i}}} \Phi\left(\mathbf{J}_{i}, \Gamma_{l}\right)=\mathbf{X}_{i}-\lambda z_{i}:=\mathbf{X}_{i}^{*},(i=1,2,3)
$$

Consequently, the $\mathbf{X}_{i}=-\nabla \frac{\mu_{i}}{T},(i=1,2,3)$ free dissipative forces causing the entropy production are not enough to describe the constrained transport processes on a membrane. Together with these free forces we have to take into account the $\lambda z_{i}$ supplementary forces, which do not cause entropy production. These forces are called thermodynamic reaction forces. Consequently, the membrane processes in fact are characterized by the

$$
\mathbf{X}_{i}^{*}:=\mathbf{X}_{i}-\lambda z_{i}=-\lambda z_{i}-\nabla \frac{\mu_{i}}{T},(i=1,2,3)
$$

thermodynamic forces.

The thermodynamic reaction forces have full analogy with the reaction forces (which do not produce work) rendered to ideal constraints in the mechanics of the mass-points. The 
thermodynamic reaction forces are similar to this having no entropy production by their actions, since

$$
\mathbf{j}_{1} \cdot z_{1} \boldsymbol{\lambda}+\mathbf{j}_{2} \cdot z_{2} \boldsymbol{\lambda}+\mathbf{j}_{3} \cdot z_{3} \boldsymbol{\lambda}=\left(z_{1} \mathbf{j}_{1}+z_{2} \mathbf{j}_{2}+z_{3} \mathbf{j}_{3}\right) \cdot \boldsymbol{\lambda}=0
$$

where we take the equation (47) into account.

It is possible to realize the constrain by an electric field inside of the membrane, which forces the zero value of the overall current density. In case of the cell-membrane this extra field is produced by the free charges on the inner and outer surface of the membrane in stationer state. When the electric field- strength is $\mathbf{E}$, and $\lambda=\mathbf{E} / T$, the force potential is calculated by inversion of equation (50) on fluxes, and substitute these into the second equation of (49). When the inverted constitutive equations are $\mathbf{J}_{i}=\mathbf{J}_{i}\left(\mathbf{X}_{j}^{*}, \Gamma_{l}\right)$, we get for the force potential

$$
\begin{aligned}
& \Psi\left(\mathbf{X}_{i}^{*}, \boldsymbol{\lambda}, \Gamma_{l}\right):=\mathbf{X}_{i} \cdot \mathbf{J}_{i}-\Phi\left(\mathbf{J}_{i}, \Gamma_{l}\right)+\boldsymbol{\lambda} \cdot\left(z_{1} \mathbf{j}_{1}+z_{2} \mathbf{j}_{2}+z_{3} \mathbf{j}_{3}\right)= \\
& =\mathbf{X}_{i}^{*} \cdot \mathbf{J}_{i}\left(\mathbf{X}_{j}^{*}, \Gamma_{l}\right)-\Phi\left(\mathbf{J}_{i}\left(\mathbf{X}_{j}^{*}, \Gamma_{l}\right), \Gamma_{l}\right)
\end{aligned}
$$

The constitutive equations for the current densities can be deduced from this potential

$$
\mathbf{J}_{i}=\nabla_{\mathbf{X}_{i}^{*}} \Psi\left(\mathbf{X}_{i}^{*}, \lambda, \Gamma_{l}\right)
$$

The Lagrange multiplier $\lambda$ in the force potential is determined from the constrain equation (47):

$$
\begin{aligned}
& z_{1} \mathbf{j}_{1}+z_{2} \mathbf{j}_{2}+z_{3} \mathbf{j}_{3}= \\
& =z_{1} \nabla_{\mathbf{X}_{1}{ }^{*}} \Psi\left(\mathbf{X}_{i}{ }^{*}, \boldsymbol{\lambda}, \Gamma_{l}\right)+z_{2} \nabla_{\mathbf{X}_{2}{ }^{*}} \Psi\left(\mathbf{X}_{i}{ }^{*}, \boldsymbol{\lambda}, \Gamma_{l}\right)+ \\
& +z_{3} \nabla_{\mathbf{X}_{3}{ }^{*}} \Psi\left(\mathbf{X}_{i}{ }^{*}, \boldsymbol{\lambda}, \Gamma_{l}\right)=0
\end{aligned}
$$

This means a solution of a non-linear equation, except the material equations are quasilinear. The task would be solved in the frame of quasi-linear Onsager constitutive theory. In this case the flux potential for isotropic membrane is

$$
\Phi\left(\mathbf{J}_{i}, \Gamma_{l}\right)=\frac{1}{2} R_{i k}\left(\Gamma_{l}\right) \mathbf{J}_{i} \cdot \mathbf{J}_{k},(i, k=1,2,3)
$$

Which modifies (50) to the case of quasi-linear constitutive equation:

$$
\mathbf{X}_{i}^{*}=R_{i k} \mathbf{j}_{k},(i, k=1,2,3)
$$

where, $\mathbf{L}=\left[L_{i k}\right]$ are the resistivity coefficients which might depend on the various intensity parameters like temperature, ionic concentrations, etc. Inverting the equations (57) by fluxes, we obtain

$$
\mathbf{j}_{i}=L_{i k} \mathbf{X}_{k}^{*}=,(i, k=1,2,3), \mathbf{L}=\left[L_{i k}\right]=\mathbf{R}^{-1}=\left[R_{i k}\right]^{-1}
$$

where $\mathbf{L}=\left[L_{i k}\right]$ is the matrix composed by the conductivity coefficients of the given membrane. With these 


$$
\begin{aligned}
& \Psi\left(\mathbf{X}_{i}{ }^{*}, \boldsymbol{\lambda}, \Gamma_{l}\right):=\mathbf{X}_{i} \cdot \mathbf{J}_{i}-\Phi\left(\mathbf{J}_{i}, \Gamma_{l}\right)+\boldsymbol{\lambda} \cdot\left(z_{1} \mathbf{j}_{1}+z_{2} \mathbf{j}_{2}+z_{3} \mathbf{j}_{3}\right)= \\
& =\mathbf{X}_{i}^{*} \cdot \mathbf{J}_{i}-\Phi\left(\mathbf{J}_{i}, \Gamma_{l}\right)=L_{i k} \mathbf{X}_{i}{ }^{*} \cdot \mathbf{X}_{k}^{*}-\frac{1}{2} R_{i k} L_{i l} \mathbf{X}_{l}{ }^{*} \cdot L_{k m} \mathbf{X}_{m}{ }^{*}=\frac{1}{2} L_{i k} \mathbf{X}_{i}{ }^{*} \cdot \mathbf{X}_{k}^{*}
\end{aligned}
$$

Determining the thermodynamic constrain-force we use the equation (47):

$$
\begin{aligned}
& z_{1} \mathbf{j}_{1}+z_{2} \mathbf{j}_{2}+z_{3} \mathbf{j}_{3}= \\
& =z_{i} \nabla_{\mathbf{X}_{i}^{*}} \Psi\left(\mathbf{X}_{i}^{*}, \lambda, \Gamma_{l}\right)=L_{i k} z_{i} \mathbf{X}_{k}^{*}=0
\end{aligned}
$$

Using equation (51) and the condition $\lambda=\mathrm{E} / T$, we get for the electric field-strength

$$
\begin{aligned}
& \mathbf{E}=-\frac{L_{1} \nabla \mu_{1}+L_{2} \nabla \mu_{2}+L_{2} \nabla \mu_{3}}{L_{1} z_{1}+L_{2} z_{2}+L_{2} z_{3}}, \\
& L_{i}:=z_{i} \sum_{k=1}^{3} L_{i k}
\end{aligned}
$$

which is necessary for a net current-free state of membrane. Consequently, the constrained transport processes of membrane by constitutive point of view can be characterized by the material equations

$$
\begin{aligned}
& \mathbf{j}_{i}=L_{i k} \mathbf{X}_{k}^{*}(i, k=1,2,3), \\
& \mathbf{X}_{i}^{*}:=\frac{1}{T} \frac{L_{1} \nabla \mu_{1}+L_{2} \nabla \mu_{2}+L_{2} \nabla \mu_{3}}{L_{1} z_{1}+L_{2} z_{2}+L_{2} z_{3}} z_{i}-\nabla \frac{\mu_{i}}{T}
\end{aligned}
$$

and the following OM-function

$$
\begin{aligned}
& o\left(\mathbf{X}_{i}^{*}, \mathbf{J}_{i}, \Gamma_{l}\right)=\Psi\left(\mathbf{X}_{i}^{*}, \Gamma_{l}\right)+\Phi\left(\mathbf{J}_{i}, \Gamma_{l}\right)-\mathbf{X}_{i}^{*} \cdot \mathbf{J}_{i}= \\
& =\frac{1}{2} L_{i k} \mathbf{X}_{i}^{*} \cdot \mathbf{X}_{k}^{*}+\frac{1}{2} R_{i k} \mathbf{J}_{i} \cdot \mathbf{J}_{k}-\mathbf{X}_{i}^{*} \cdot \mathbf{J}_{i}
\end{aligned}
$$

The above method could be applied to other biologically important problems like ionselective membranes, semi-permeable membranes, etc. The above example shows, that constraints (at least when they are linear) do not destroy the symmetry relations.

\subsection{Exercises}

1.6.1. Show the product of $L_{i k}$ (conductivity matrix) and $R_{i k}$ (resistivity matrix) in linear Onsager constitutive theory is the unit matrix.

1.6.2. Show that the flux and force potentials in linear Onsager theory are strictly convex functions.

1.6.3. Prove the Donkin's theorem based on Exercise 1.

1.6.4. Show the product of Hessian matrices of dissipation potentials is the unit matrix.

1.6.5. Determine the thermodynamic reaction forces for a membrane having multiple transports of chemical components forced by a current density from outside source. Determine the OM-function of the process. What is the entropy production resulted by the reaction forces in this case?

1.6.6. Determine the linear constitutive equations of a semi-permeable membrane. 


\section{Minimum principle of steady-state system in case of vector processes. Deduction of the non-homogeneous transport equations from variational principle}

Let us consider a system having $N$ vector-processes with $\mathbf{j}_{i}$ and $\nabla \Gamma_{i}$ thermodynamic fluxes and forces. Study these in the frame of the generalized Onsager constitutive theory. Consequently the flux of the i-th vector process can be derived from the strictly convex $\Psi\left(\nabla \Gamma_{j}, \Gamma_{k}\right)$ force dissipation potential

$$
\mathbf{j}_{i}=\frac{\partial \Psi}{\partial \nabla \Gamma_{i}},(i=1,2, \ldots, N)
$$

Substituting this constitutive equation of i-th flux into the stationer balance equation (4) of ith extensive

$$
\nabla \cdot \mathbf{j}_{i}=\sigma_{i},(i=1,2, \ldots, N)
$$

we get the following system of partial differential equations on intensive parameters

$$
\nabla \cdot \frac{\partial \Psi\left(\nabla \Gamma_{j}, \Gamma_{k}\right)}{\partial \nabla \Gamma_{i}}=\sigma_{i},(i=1,2, \ldots, N)
$$

which are the inhomogeneous transport equations of the steady-state thermodynamic system. To generalize of the task, here we introduced the source $\sigma_{i}$, supposing their prescribed continuous dependence from the space-coordinates. Such source could be for example in heat-conduction process when the system is placed in a radiation field, and absorbs energy from that in all of its volume; or in case of the electric conduction of a semiconductor, in which the radiation field generates electron-hole pairs. It is evident that the equations (64) and (65) are equivalent with (66). Let the boundary of the system be $\Omega$, and order for every transport process one $\Omega_{\Gamma_{j}}, \Omega_{\mathbf{j}_{j}}$ surface partition such as: $\Omega=\Omega_{\Gamma_{j}} \cup \Omega_{\mathfrak{j}_{j}}, \Omega_{\Gamma_{j}} \cap \Omega_{\mathbf{j}_{j}}=\varnothing$. Let's give time-independent mixed boundary conditions for partial differential equations (66). This means that we use Dirichlet boundary condition for $\Gamma_{j}$ 's on $\Omega_{\Gamma_{j}}$ 's and a Neumann boundary condition for $\mathbf{j}_{j}$ 's on $\Omega_{\mathbf{j}_{j}}$ 's, i.e.

$$
\begin{aligned}
& \Gamma_{j}=\Gamma_{j}^{*} \text { on } \Omega_{\Gamma_{j}} \\
& \mathbf{j}_{j n}=j_{j}^{*} \text { on } \Omega_{\mathbf{j}_{j}},(j=1,2, \ldots, N)
\end{aligned}
$$

where $\Gamma_{j}^{*}$ and $j_{j}^{*}$ are given functions defined on those partitions of the boundary and $\mathbf{j}_{j n}$ is the normal coordinate of the $j$-th flux on the boundary.

Let the $\Gamma_{j}$ intensives be two times and the $\mathbf{j}_{j}$ fluxes be continuously differentiable functions with respect to the space coordinates in all the points of the system, i.e. $\Gamma_{j} \in C^{2}(V), \mathbf{j}_{j} \in C^{1}(V),(j=1,2, \ldots, N)$, where $V$ is the region occuppied by the system. 
Suppose that the transport equations (66) have a unique solution. Furthermore, we use the plausible equivalence of the equations (64) and (65) with (66). As we showed above, the OMfunction satisfies the Young-inequality in any $\left(\mathbf{j}_{1}, \ldots, \mathbf{j}_{N}, \Gamma_{1}, \ldots, \Gamma_{N}\right)$ ordered vector-scalar $2 N$ sets, so

$$
o\left(\nabla \Gamma_{j}, \mathbf{j}_{j}, \Gamma_{k}\right)=\Phi\left(\mathbf{j}_{j}, \Gamma_{k}\right)+\Psi\left(\nabla \Gamma_{j}, \Gamma_{k}\right)-\mathbf{j}_{i} \cdot \nabla \Gamma_{i} \geq 0
$$

where the equality in (68) is satisfied only when the constitutive equations expressed by thermodynamic forces:

$$
\nabla \Gamma_{i}=\frac{\partial \Phi\left(\mathbf{j}_{j}, \Gamma_{k}\right)}{\partial \mathbf{j}_{i}},(i=1,2, \ldots, N)
$$

are valid together with (64).

This local minimum principle could be extended to global by integration of OM-function into the region $V$ of the system:

$$
\int_{V} o\left(\nabla \Gamma_{j}, \mathbf{j}_{j}, \Gamma_{k}\right) d V=\int_{V}\left[\Phi\left(\mathbf{j}_{j}, \Gamma_{k}\right)+\Psi\left(\nabla \Gamma_{j}, \Gamma_{k}\right)-\mathbf{j}_{i} \cdot \nabla \Gamma_{i}\right] d V \geq 0
$$

This functional will be minimal, (i.e. zero) when the (64), (65) generalized Onsager constitutive equations are fulfilled. Using the Gauss's divergence theorem on the total entropy production, we obtain

$$
\int_{V} \mathbf{j}_{i} \cdot \nabla \Gamma_{i} d V=\int_{V} \nabla \cdot\left(\mathbf{j}_{i} \Gamma_{i}\right) d V-\int_{V} \Gamma_{i} \nabla \cdot \mathbf{j}_{i} d V=\oint_{\Omega}\left(\mathbf{j}_{i} \Gamma_{i}\right)_{n} d A-\int_{V} \Gamma_{i} \sigma_{i} d V
$$

where the $n$-index denotes the coordinates in direction of the outer normal vector of $\Omega$ and we used the stationer balance equations (65). With these the global OM-function of the steady-state system is

$$
\begin{aligned}
& O\left(\nabla \Gamma_{j}, \mathbf{j}_{j}, \Gamma_{k}\right):=\int_{V} o\left(\nabla \Gamma_{j}, \mathbf{j}_{j}, \Gamma_{k}\right) d V= \\
& =\int_{V}\left[\Gamma_{i} \sigma_{i}+\Phi\left(\mathbf{j}_{j}, \Gamma_{k}\right)+\Psi\left(\nabla \Gamma_{j}, \Gamma_{k}\right)\right] d V-\oint_{\Omega}\left(\mathbf{j}_{i} \Gamma_{i}\right)_{n} d A \geq 0
\end{aligned}
$$

Let's construct a conditional extremum principle with this functional. To do this, we shall introduce the boundary conditions (67) into the above OM-functional, then we get

$$
\begin{aligned}
& O\left(\nabla \Gamma_{j}, \mathbf{j}_{j}, \Gamma_{k}\right)=\int_{V}\left[\sigma_{i} \Gamma_{i}+\Phi\left(\mathbf{j}_{j}, \Gamma_{k}\right)+\Psi\left(\nabla \Gamma_{j}, \Gamma_{k}\right)\right] d V+ \\
& -\int_{\Omega_{\Gamma_{j}}} j_{j n} \Gamma_{j}^{*} d A-\int_{\Omega_{j_{j}}} j_{j}^{*} \Gamma_{j} d A
\end{aligned}
$$

and let its definition domain be 


$$
D(O)=\left\{\begin{array}{l}
\left(\mathbf{j}_{1}, ., \mathbf{j}_{j}, ., \mathbf{j}_{N}, \Gamma_{1}, ., \Gamma_{j}, \Gamma_{N}\right) \in \mid \nabla \cdot \mathbf{j}_{j}=\sigma_{j}, \Gamma_{j}=\Gamma_{j}^{*} \text { on } \Omega_{\Gamma_{j}} \\
j_{j n}=j_{j}^{*} \text { on } \Omega_{\mathbf{j}_{j}}
\end{array}\right\}
$$

Also the admissible intensive parameters are all of the $C^{2}(V)$ functions which have prescribed boundary values. As well as the admissible fluxes all of the $C^{1}(V)$ vector functions which satisfy the stationer balance equations (65) belong to them and their normal coordinates are prescribed on the boundary.

Now, we can see that exclusive restrictions were used only for thermodynamic fluxes, namely they have to satisfy the steady-state balance equations. It is easy to see that $D(O)$ is not empty, and contains the unique solutions $\left(\mathbf{j}_{10}, . ., \mathbf{j}_{i 0}, . ., \mathbf{j}_{N 0}, \Gamma_{10}, . ., \Gamma_{k 0}, . ., \Gamma_{N 0}\right)$ of the steady-state transport equations. Now we can formulate the minimum principle of the steady-state system.

Theorem 1. The $\left(\mathbf{j}_{10}, . ., \mathbf{j}_{i 0}, . ., \mathbf{j}_{N 0}, \Gamma_{10}, . ., \Gamma_{k 0}, . ., \Gamma_{N 0}\right)$ ordered vector-scalar $2 N$ set is the solution of the system of transport equations (64), (65) if and only if it minimizes the OMfunctional.

$$
O\left(\nabla \Gamma_{j 0}, \mathbf{j}_{j 0}, \Gamma_{k 0}\right)<O\left(\nabla \Gamma_{j}, \mathbf{j}_{j}, \Gamma_{k}\right), \forall\left(\mathbf{j}_{1}, ., \mathbf{j}_{j}, ., \mathbf{j}_{N}, \Gamma_{1}, . ., \Gamma_{j}, \Gamma_{N}\right) \in D(O)
$$

Proof. First, we show that if $\left(\mathbf{j}_{10}, . ., \mathbf{j}_{i 0}, . ., \mathbf{j}_{N 0}, \Gamma_{10}, . ., \Gamma_{k 0}, . ., \Gamma_{N 0}\right) \in D(O)$ is solution of the equations (64) and (65) then it minimizes the OM-functional (73). To do this, add and deduct the entropy production to the integrand of (73), and apply the Gauss's divergence theorem like

$$
\begin{aligned}
& \int_{V} \mathbf{j}_{j} \cdot \nabla \Gamma_{j} d V=-\int_{V} \nabla \cdot \mathbf{j}_{j} \Gamma_{j} d V+\oint_{\Omega} \mathbf{j}_{j} \Gamma_{j} \cdot d \mathbf{A}= \\
& =-\int_{V} \nabla \cdot \mathbf{j}_{j} \Gamma_{j} d V+\int_{\Omega_{\Gamma_{j}}} j_{j n} \Gamma_{j}^{*} d A+\int_{\Omega_{\mathrm{j}_{j}}} j_{j}^{*} \Gamma_{j} d A
\end{aligned}
$$

In this case we obtain

$$
\begin{aligned}
& O\left(\nabla \Gamma_{j}, \mathbf{j}_{j}, \Gamma_{k}\right)=\int_{V}\left[\left(\sigma_{i}-\nabla \cdot \mathbf{j}_{i}\right) \Gamma_{i}+\left(\Phi+\Psi-\mathbf{j}_{j} \cdot \nabla \Gamma_{j}\right)\right] d V= \\
& =\int_{V}\left[\left(\Phi+\Psi-\mathbf{j}_{j} \cdot \nabla \Gamma_{j}\right)\right] d V \geq 0
\end{aligned}
$$

where we used the fact that the fluxes satisfy the (65) stationer balance equations. Due to (70) this expression is always positive on $D(O)$, and it has its minimum when the constitutive equations are obtained, i.e. at the solution of the transport equations. Hence at the solution of the transport equations $O\left(\nabla \Gamma_{j}, \mathbf{j}_{j}, \Gamma_{k}\right)$ has its minimum.

Second, we show that the necessary conditions of the minimum of the OM-functional on $D(O)$ are identical with the transport equations. For this we introduce the admissible variations. The

$$
\left(\mathbf{j}_{10}+\delta \mathbf{j}_{1}, . ., \mathbf{j}_{i 0}+\delta \mathbf{j}_{i}, . . \mathbf{j}_{N 0}+\delta \mathbf{j}_{N}, \Gamma_{10}+\delta \Gamma_{1}, . ., \Gamma_{k 0}+\delta \Gamma_{k} . ., \Gamma_{N 0}+\delta \Gamma_{N}\right)
$$


ordered vector-scalar $2 N$ sets are called varied elements of $D(O)$, when the variations $\left(\delta \mathbf{j}_{1}, . ., \delta \mathbf{j}_{i}, . ., \delta \mathbf{j}_{N}\right)$ of fluxes satisfy the homogeneous stationer balance equations:

$$
\nabla \cdot \delta \mathbf{j}_{i}=0,(i=1,2, \ldots, N)
$$

and they are zero on $\Omega_{\mathbf{j}_{j}}$ 's as well as the $\left(\delta \Gamma_{1}, ., \delta \Gamma_{j} ., \delta \Gamma_{N}\right)$ variations of intensives are all of the $C^{2}(V)$ functions, which are zero on $\Omega_{\Gamma_{j}}$ 's. Then the first variation of OM- functional (73) is

$$
\begin{aligned}
& \delta O=\int_{V}\left[\left(\sigma_{i}-\left.\nabla \cdot \frac{\partial \Psi}{\partial \nabla \Gamma_{i}}\right|_{\Gamma_{k 0}, \nabla \Gamma_{j 0}}+\left.\frac{\partial(\Psi+\Phi)}{\partial \Gamma_{i}}\right|_{\Gamma_{k 0}, \nabla \Gamma_{j 0}, j_{j 0}}\right) \delta \Gamma_{i}+\left.\frac{\partial \Phi}{\partial \mathbf{j}_{i}}\right|_{\Gamma_{k 0}, \mathbf{j}_{j 0}} \cdot \delta \mathbf{j}_{i}\right] d V= \\
& =\int_{V}\left[\left(\sigma_{i}-\left.\nabla \cdot \frac{\partial \Psi}{\partial \nabla \Gamma_{i}}\right|_{\Gamma_{k 0}, \nabla \Gamma_{j 0}}\right) \delta \Gamma_{i}+\left.\frac{\partial \Phi}{\partial \mathbf{j}_{i}}\right|_{\Gamma_{k 0}, \mathbf{j}_{j 0}} \cdot \delta \mathbf{j}_{i}\right] d V=0
\end{aligned}
$$

where we take Donkin's theorem into account (36), which states that the variations of the sum of dissipation potentials with respect to the intensive parameters are zero. The necessary condition of the extremum of a functional is that its first variation vanishes. Since the variations of intensive parameters $\delta \Gamma_{i}=0,(i=1,2,3, \ldots, N)$ are arbitrary and independent of the variation of fluxes, it is possible to choose $\delta \Gamma_{i}=0,(i=1,2,3, \ldots, N)$, then we get:

$$
\int_{V}\left[\left.\frac{\partial \Phi}{\partial \mathbf{j}_{i}}\right|_{\Gamma_{k 0}, \mathbf{j}_{j 0}} \cdot \delta \mathbf{j}_{i}\right] d V=0,(i=1,2, \ldots, N)
$$

with these from (80) follows

$$
\delta O=\int_{V}\left(\sigma_{i}-\left.\nabla \cdot \frac{\partial \Psi}{\partial \nabla \Gamma_{i}}\right|_{\Gamma_{k 0}, \nabla \Gamma_{j 0}}\right) \delta \Gamma_{i} d V=0
$$

From this and the fundamental lemma of variational calculus, we obtain the transport equations (66)

$$
\sigma_{i}-\nabla \cdot \frac{\partial \Psi}{\partial \nabla \Gamma_{i}}=0,(i=(1,2, \ldots, N)
$$

because the variations of intensives are arbitrary.

Consequently, the first group of the necessary conditions of the extremum of the OMfunctional is equivalent with the transport equations. To discuss the second group of the necessary conditions, which is represented in a form of functional, consider the following vector analytical

$$
\begin{aligned}
& \int_{V}^{[}\left[\left.\frac{\partial \Phi}{\partial \mathbf{j}_{i}}\right|_{\Gamma_{k 0}, \mathbf{j}_{j 0}} \cdot \delta \mathbf{j}_{i}\right] d V=\int_{V}\left[\nabla \Gamma_{i 0} \cdot \delta \mathbf{j}_{i}\right] d V=\int_{V} \nabla \cdot\left(\Gamma_{i 0} \delta \mathbf{j}_{i}\right) d V- \\
& -\int_{V} \Gamma_{i 0} \nabla \cdot \delta \mathbf{j}_{i} d V=\oint_{A} \Gamma_{i 0} \delta \mathbf{j}_{i} \cdot d \mathbf{A}-\int_{V} \Gamma_{i 0} \nabla \cdot \delta \mathbf{j}_{i} d V=0
\end{aligned}
$$


identity transform, where we used Gauss's divergence theorem. Now, we see that the second group of necessity condition obtained the constitutive equations (69) and the conditions for that reason, because the variations (79) for the zero-values of the fluxes are source-free and are zero on the boundary.

Short formulation. To keep the further discussion simple, let us assume that thermodynamic forces and fluxes are square-integrable scalar or vector functions, respectively. In this case these functions form a Hilbert space, where the scalar-product can be introduced by volume integral. Denote this space by $H$, and the scalar-product by the brackets $\langle$,$\rangle . The solutions of the homogeneous balance equations (79) also form Hilbert$ space too, which is a sub-space in $H\left[{ }^{18}\right]$. Denote this sub-place with $H_{1}$, and its orthogonal complement in $H$ with $H_{2}$, so $H=H_{1} \oplus H_{2}$. In this case any $\mathbf{w} \in H$ can be given in the form $\mathbf{w}=\mathbf{u}+\mathbf{v}$, where $\mathbf{u} \in H_{1}, \mathbf{v} \in H_{2}$ and $\langle\mathbf{u}, \mathbf{v}\rangle=0$. This means that $\mathbf{u}$ and $\mathbf{v}$ are orthogonal with each other. In the light of this, the second group of the necessity of the extremum can be interpreted that in a steady-state system the quantities

$$
\left.\frac{\partial \Phi}{\partial \mathbf{j}_{i}}\right|_{\Gamma_{k 0}, \mathbf{j}_{j 0}},(i=1,2, \ldots, N)
$$

which are the thermodynamic forces and to this canonically conjugated thermodynamic flux variations $\delta \mathbf{j}_{i}$ satisfying the homogeneous balance equations (79), are orthogonal, so

$$
\left\langle\nabla \Gamma_{i 0}=\left.\frac{\partial \Phi}{\partial \mathbf{j}_{i}}\right|_{\Gamma_{k 0}, \mathbf{j}_{j 0}}, \delta \mathbf{j}_{i}\right\rangle=0, \forall \delta \mathbf{j}_{i} \in H_{1},(i=1,2, \ldots, N)
$$

Hence

$$
\nabla \Gamma_{i 0}=\left.\frac{\partial \Phi}{\partial \mathbf{j}_{i}}\right|_{\Gamma_{k 0}, \mathbf{j}_{j 0}} \in H_{2},(i=1,2, \ldots, N)
$$

This property was proven directly in (84), and we prove it again with the new formalism regarding the further considerations. To proceed in our study the $\left\langle\nabla \Gamma_{i}, \delta \mathbf{j}_{i}\right\rangle$ scalar-product where $\delta \mathbf{j}_{i}$ disappears at the boundary of the system. It is easy to show with the help of the definition of the inner product and by the Gauss's divergence theorem, that

$$
\left\langle\nabla \Gamma_{i}, \delta \mathbf{j}_{i}\right\rangle=\left\langle\Gamma_{i},-\nabla \cdot \delta \mathbf{j}_{i}\right\rangle
$$

Also, the adjoint of the gradient operator is equally of the minus of the divergence operator. Due to the variation of fluxes satisfy the homogeneous stationer balance equations, we get from (88) the ortogonality is described by (87):

$$
\left\langle\nabla \Gamma_{i}, \delta \mathbf{j}_{i}\right\rangle=\left\langle\Gamma_{i},-\nabla \cdot \delta \mathbf{j}_{i}\right\rangle=0
$$

Finally, we can construct from the above restricted extremum principle a free extremumtask by Lagrange's method of multipliers. This free variational principle is the following 


$$
\begin{aligned}
& O\left(\mathbf{j}_{j}, \nabla \mathbf{j}_{j}, \nabla \Gamma_{j}, \Gamma_{k}, \lambda_{i}\right)= \\
& =\int_{V} L\left(\mathbf{j}_{j}, \nabla \mathbf{j}_{j}, \nabla \Gamma_{j}, \Gamma_{k}, \lambda_{i}\right) d V-\int_{\Omega_{\Gamma_{j}}} j_{j n} \Gamma_{j}^{*} d A-\int_{\Omega_{\mathrm{j}_{j}}} j_{j}^{*} \Gamma_{j} d A=\min \\
& L\left(\mathbf{j}_{j}, \nabla \mathbf{j}_{j}, \nabla \Gamma_{j}, \Gamma_{k}, \lambda_{i}\right)=\sigma_{i} \Gamma_{i}+\Phi\left(\mathbf{j}_{j}, \Gamma_{k}\right)+\Psi\left(\nabla \Gamma_{j}, \Gamma_{k}\right)+\lambda_{i}\left(\nabla \cdot \mathbf{j}_{i}-\sigma_{i}\right)
\end{aligned}
$$

The Euler-Lagrange equations belong to (90) follows by the free variations of intensive parameters, fluxes and multipliers, i.e.

$$
\begin{aligned}
& \frac{\partial L}{\partial \Gamma_{i}}-\nabla \cdot \frac{\partial L}{\partial \nabla \Gamma_{i}}=\sigma_{i}-\nabla \cdot \frac{\partial \Psi}{\partial \nabla \Gamma_{i}}=0, \frac{\partial L}{\partial \mathbf{j}_{i}}-\nabla \frac{\partial L}{\partial \nabla \cdot \mathbf{j}_{i}}=\frac{\partial \Phi}{\partial \mathbf{j}_{i}}-\nabla \lambda_{i}=0 \\
& \frac{\partial L}{\partial \lambda_{i}}=\nabla \cdot \mathbf{j}_{i}-\sigma_{i}=0,(i=1,2, \ldots, N)
\end{aligned}
$$

from where the firsts are stationer transport equations, the seconds are constitutive equations expressing the connections between the forces and currents, while the thirds are the stationer balance equations. Now we see that it is possible to choose the $\lambda_{i}$ multipliers as the intensive parameters $\Gamma_{i}$ of the steady-state system, because the constitutive equations $\nabla \Gamma_{i}=\frac{\partial \Phi}{\partial \mathbf{j}_{i}}$ are fulfilled. The boundary conditions can be obtained from the surface terms by detailed calculations

$$
\begin{aligned}
& \left.\frac{\partial \Psi}{\partial \nabla \Gamma_{i}}\right|_{n}=j_{j}^{*} \text { on } \Omega_{\mathbf{j}_{j}} \\
& \Gamma_{i}=\Gamma_{i}^{*} \text { on } \Omega_{\Gamma_{j}},(i=1,2, \ldots, N)
\end{aligned}
$$

It is evident that the above formulated free variational principle is a direct consequence of the restricted minimum principle, which is formulated in the Theorem 1 . We refer to both of these principles as minimum principle of steady-state system (MPSTS). This principle states the minimum of OM-functional for the real processes of a steady-state thermodynamic system, also which satisfy the balance equations and the constitutive equations as well.

\subsection{Minimum principle of global entropy production. Deduction of the homogeneous steady-state transport equations from variational principle}

In following we shall show that in case of homogeneous steady-state balance equations the MPSTS guarantees the minimum of the global entropy production.

In case of source-free balance equations the homogeneous transport equations of a steadystate system can be obtained by the substitution of the generalized Onsager's constitutive equations

$$
\mathbf{j}_{i}=\frac{\partial \Psi}{\partial \nabla \Gamma_{i}},(i=1,2, \ldots, N)
$$

into the homogeneous steady-state balance equations using the of the vector-fluxes (4) 


$$
\nabla \cdot \mathbf{j}_{i}=0,(i=1,2, \ldots, N)
$$

then we get the system of the homogenous stationer transport equations

$$
\nabla \cdot \frac{\partial \Psi\left(\nabla \Gamma_{j}, \Gamma_{k}\right)}{\partial \nabla \Gamma_{i}}=0,(i=1,2, \ldots, N)
$$

Consider the earlier fixed (67) boundary conditions for these partial differential equations and start again with the OM-function of the generalized Onsager constitutive theory to explain the principle of minimal entropy production

$$
o\left(\nabla \Gamma_{j}, \mathbf{j}_{j}, \Gamma_{k}\right)=\Phi\left(\mathbf{j}_{j}, \Gamma_{k}\right)+\Psi\left(\nabla \Gamma_{j}, \Gamma_{k}\right)-\mathbf{j}_{i} \cdot \nabla \Gamma_{i} \geq 0
$$

Considering the disappearance of the divergences of fluxes by (94), reformulate (96):

$$
\begin{aligned}
& o\left(\nabla \Gamma_{j}, \mathbf{j}_{j}, \Gamma_{k}\right)=\Phi\left(\mathbf{j}_{j}, \Gamma_{k}\right)+\Psi\left(\nabla \Gamma_{j}, \Gamma_{k}\right)-\mathbf{j}_{i} \cdot \nabla \Gamma_{i}= \\
& =\Phi\left(\mathbf{j}_{j}, \Gamma_{k}\right)+\Psi\left(\nabla \Gamma_{j}, \Gamma_{k}\right)-\nabla \cdot\left(\mathbf{j}_{i} \Gamma_{i}\right) \geq 0
\end{aligned}
$$

The integral of the OM-function on the $V$ volume of the system gives the global OMfunction of the steady-state system

$$
\begin{aligned}
& O\left(\nabla \Gamma_{j}, \mathbf{j}_{j}, \Gamma_{k}\right):=\int_{V} o\left(\nabla \Gamma_{j}, \mathbf{j}_{j}, \Gamma_{k}\right) d V= \\
& =\int_{V}\left[\Phi\left(\mathbf{j}_{j}, \Gamma_{k}\right)+\Psi\left(\nabla \Gamma_{j}, \Gamma_{k}\right)\right] d V-\oint_{\Omega}\left(\mathbf{j}_{i} \Gamma_{i}\right)_{n} d A \geq 0
\end{aligned}
$$

Introducing the boundary values of intensive parameters to this global OM-function by the method used in the previous paragraph, we get the final form of the OM-functional

$$
\begin{aligned}
& O\left(\nabla \Gamma_{j}, \mathbf{j}_{j}, \Gamma_{k}\right):=\int_{V}\left[\Phi\left(\mathbf{j}_{j}, \Gamma_{k}\right)+\Psi\left(\nabla \Gamma_{j}, \Gamma_{k}\right)\right] d V+ \\
& -\int_{\Omega_{\Gamma_{j}}} j_{j n} \Gamma_{j}^{*} d A-\int_{\Omega_{\mathrm{j}_{j}}} j_{j}^{*} \Gamma_{j} d A
\end{aligned}
$$

Let its definition domain be

$$
D_{0}(O)=\left\{\begin{array}{l}
\left(\mathbf{j}_{1}, ., \mathbf{j}_{j}, ., \mathbf{j}_{N}, \Gamma_{1}, ., \Gamma_{j}, \Gamma_{N}\right) \in \mid \nabla \cdot \mathbf{j}_{j}=0, \Gamma_{j}=\Gamma_{j}^{*} \text { on } \Omega_{\Gamma_{j}} \\
j_{j n}=j_{j}^{*} \text { on } \Omega_{\mathbf{j}_{j}}
\end{array}\right\}
$$

where the admissible intensive parameters are all the $C^{2}(V)$ functions which have definite boundary values, the admissible fluxes are all the $C^{1}(V)$ vector-functions which satisfy the source-free stationer balance equations and their normal coordinates are prescribed on the boundary. Consider the $\left(\mathbf{j}_{10}, . ., \mathbf{j}_{i 0}, . ., \mathbf{j}_{N 0}, \Gamma_{10}, . ., \Gamma_{k 0}, . ., \Gamma_{N 0}\right)$ ordered vector-scalar $2 N$ set as the solution of equations (93) and (94), which are tantamount with the transport equations (95). Now we can formulate the minimum principle of the steady-state system in case of sourcefree balance equations. 
Theorem 2. The $\left(\mathbf{j}_{10}, . ., \mathbf{j}_{i 0}, . ., \mathbf{j}_{N 0}, \Gamma_{10}, . ., \Gamma_{k 0}, . ., \Gamma_{N 0}\right)$ ordered vector-scalar $2 N$ set is the solution of the system of the transport equations (93), (94), if and only if when it minimizes the OM-functional.

$$
O\left(\nabla \Gamma_{j 0}, \mathbf{j}_{j 0}, \Gamma_{k 0}\right)<O\left(\nabla \Gamma_{j}, \mathbf{j}_{j}, \Gamma_{k}\right), \forall\left(\mathbf{j}_{1}, ., \mathbf{j}_{j}, ., \mathbf{j}_{N}, \Gamma_{1}, . ., \Gamma_{j}, \Gamma_{N}\right) \in D_{0}(O)
$$

To prove this, add and deduct the entropy production to the integrand of (99), and apply the Gauss's divergence theorem like

$$
\begin{aligned}
& O\left(\nabla \Gamma_{j}, \mathbf{j}_{j}, \Gamma_{k}\right)=\int_{V}\left[-\nabla \cdot \mathbf{j}_{i} \Gamma_{i}+\left(\Phi+\Psi-\mathbf{j}_{j} \cdot \nabla \Gamma_{j}\right)\right] d V= \\
& =\int_{V}\left[\left(\Phi+\Psi-\mathbf{j}_{j} \cdot \nabla \Gamma_{j}\right)\right] d V \geq 0
\end{aligned}
$$

where we used the fact that the fluxes satisfy the (94) homogeneous balance equations. This expression is always positive on $D_{0}(O)$, and it has its minimum when the constitutive equations are valid as well, i.e. at the solution of the transport equations. Hence the solution of the transport equations is minimum place of $O\left(\nabla \Gamma_{j}, \mathbf{j}_{j}, \Gamma_{k}\right)$. We can prove that a part of the necessary conditions of the minimum are identical with the transport equations. For this we define the

$$
\left(\mathbf{j}_{10}+\delta \mathbf{j}_{1}, . ., \mathbf{j}_{i 0}+\delta \mathbf{j}_{i}, . . \mathbf{j}_{N 0}+\delta \mathbf{j}_{N}, \Gamma_{10}+\delta \Gamma_{1}, . ., \Gamma_{k 0}+\delta \Gamma_{k} . ., \Gamma_{N 0}+\delta \Gamma_{N}\right)
$$

vector-scalar ordered $2 N$ sets as admissible variations, when the flux $\left(\delta \mathbf{j}_{1}, . ., \delta \mathbf{j}_{i}, . ., \delta \mathbf{j}_{N}\right)$ satisfy the homogeneous stationer balance equations

$$
\nabla \cdot \delta \mathbf{j}_{i}=0,(i=1,2, \ldots, N)
$$

and they are zero on the boundaries, as well as the $\left(\delta \Gamma_{1}, ., \delta \Gamma_{j}, \delta \Gamma_{N}\right)$ variations of intensives are two times continuously derivable function with zero values on the boundaries. Then the first variation of (99) is

$$
\delta O=\int_{V}\left[\left(-\left.\nabla \cdot \frac{\partial \Psi}{\partial \nabla \Gamma_{i}}\right|_{\Gamma_{k 0}, \nabla \Gamma_{j 0}}\right) \delta \Gamma_{i}+\left.\frac{\partial \Phi}{\partial \mathbf{j}_{i}}\right|_{\Gamma_{k 0}, \mathbf{j}_{j 0}} \cdot \delta \mathbf{j}_{i}\right] d V=0
$$

where we take the Donkin's theorem into account, which states the zero value of the variation of the sum of dissipation potentials by intensive parameters and that the necessary condition of the extremum of the functional is the zero value of its first variation.

Due to the variations of intensives are independent from variations of fluxes, we can choose $\delta \Gamma_{i}=0,(i=1,2,3, \ldots, N)$, then from (105) follows

$$
\int_{V}\left[\left.\frac{\partial \Phi}{\partial \mathbf{j}_{i}}\right|_{\Gamma_{k 0}, \mathbf{j}_{j 0}} \cdot \delta \mathbf{j}_{i}\right] d V=0,(i=(1,2, \ldots, N)
$$


Consequently we get from (105):

$$
\delta O=\int_{V}\left(-\left.\nabla \cdot \frac{\partial \Psi}{\partial \nabla \Gamma_{i}}\right|_{\Gamma_{k 0}, \nabla \Gamma_{j 0}}\right) \delta \Gamma_{i} d V=0
$$

from where we obtain the system of the transport equations (95)

$$
\nabla \cdot \frac{\partial \Psi}{\partial \nabla \Gamma_{i}}=0,(i=(1,2, \ldots, N)
$$

because the variations of the intensives are arbitrary.

Consequently, one group of the necessary conditions of the extremum is the group of the transport equations. The second group, which is formulated in functional form, were discussed above.

Due to the Lagrangian of the functional (99) is the sum of the dissipation potentials, which is equal to the entropy production in case of every real steady-state physical processes, this extremum theorem involves the minimum principle of global entropy production (MPGEP). The physical meaning of MPGEP needs a clarification. Consider the variations of the fluxes and of the intensive parameters as fluctuations of the system around their stationer state values. When these fluctuations are small, the fluctuation of the global entropy production of the system is equal to its first approximations and it has a form

$$
\delta P:=\left\langle\nabla \Gamma_{i 0}+\delta \nabla \Gamma_{i}, \mathbf{j}_{i 0}+\delta \mathbf{j}_{i}\right\rangle-\left\langle\nabla \Gamma_{i 0}, \mathbf{j}_{i 0}\right\rangle \cong\left\langle\nabla \Gamma_{i 0}, \delta \mathbf{j}_{i}\right\rangle+\left\langle\delta \nabla \Gamma_{i}, \mathbf{j}_{i 0}\right\rangle
$$

The first term of the fluctuation of entropy production is zero due to the (86) ortogonality conditions, while the second term, using the

$$
\delta P \cong\left\langle\delta \nabla \Gamma_{i}, \mathbf{j}_{i 0}\right\rangle=\left\langle\delta \Gamma_{i},-\nabla \cdot \mathbf{j}_{i 0}\right\rangle
$$

transformation, will also be zero due to the source free balance equation (94). Consequently in first approximation the global entropy production has no fluctuation, so the necessary condition of the minimum of the global entropy production is fulfilled. The sufficient conditions also have to be proved. The sum of the dissipation potentials in case when the forces and fluxes are satisfied the transport- and constitutive equations is equal to the entropy production, due to the minimum of the OM-functional Proceed from (36). Due to this fact we could study the sum of the dissipation potentials instead of the entropy production. Regarding the Donkin's theorem, the second term of the Taylor series at the stationer state will be

$$
\begin{aligned}
& \delta P=\int_{V}\left[\Phi\left(\mathbf{j}_{j 0}+\delta \mathbf{j}_{j}, \Gamma_{k 0}+\delta \Gamma_{k}\right)+\Psi\left(\nabla \Gamma_{j 0}+\delta \nabla \Gamma_{j 0}, \Gamma_{k 0}+\delta \Gamma_{k}\right)\right] d V= \\
& =\int_{V}\left[\Phi\left(\mathbf{j}_{j 0}, \Gamma_{k 0}\right)+\Psi\left(\nabla \Gamma_{j 0}, \Gamma_{k 0}\right)\right] d V+\left\langle\left.\frac{\partial \Phi}{\partial \mathbf{j}_{i}}\right|_{\Gamma_{k 0}, \mathbf{j}_{j 0}}, \delta \mathbf{j}_{i}\right\rangle+\left\langle\left.\frac{\partial \Psi}{\partial \nabla \Gamma_{i}}\right|_{\Gamma_{k 0}, \mathbf{j}_{j 0}}, \delta \nabla \Gamma_{i}\right\rangle+ \\
& +\left\langle\left.\frac{\partial^{2} \Phi}{\partial \mathbf{j}_{j} \partial \mathbf{j}_{i}}\right|_{\Gamma_{k 0}, \mathbf{j}_{j 0}} \delta \mathbf{j}_{j}, \delta \mathbf{j}_{i}\right\rangle+\left\langle\left.\frac{\partial^{2} \Psi}{\partial \nabla \Gamma_{j} \partial \nabla \Gamma_{i}}\right|_{\Gamma_{k 0}, \mathbf{j}_{j 0}} \delta \nabla \Gamma_{j}, \delta \nabla \Gamma_{i}\right\rangle
\end{aligned}
$$


When $\delta^{(1)} P$ is the linear part of the variations of entropy production (first variation) and the quadratic part is the $\delta^{(2)} P$ (second variation), then (111) will have a form

$$
\begin{aligned}
& \delta P=\delta^{(1)} P+\delta^{(1)} P, \delta^{(1)} P=\left\langle\left.\frac{\partial \Phi}{\partial \mathbf{j}_{i}}\right|_{\Gamma_{k 0}, \mathbf{j}_{j 0}}, \delta \mathbf{j}_{i}\right\rangle+\left\langle\left.\frac{\partial \Psi}{\partial \nabla \Gamma_{i}}\right|_{\Gamma_{k 0}, \mathbf{j}_{j 0}}, \delta \nabla \Gamma_{i}\right\rangle= \\
& =\left\langle\left.\frac{\partial \Phi}{\partial \mathbf{j}_{i}}\right|_{\Gamma_{k 0}, \mathbf{j}_{j 0}}, \delta \mathbf{j}_{i}\right\rangle+\left\langle-\left.\nabla \cdot \frac{\partial \Psi}{\partial \nabla \Gamma_{i}}\right|_{\Gamma_{k 0}, \mathbf{j}_{j 0}}, \delta \Gamma_{i}\right\rangle \\
& \delta^{(2)} P=\left\langle\left.\frac{\partial^{2} \Phi}{\partial \mathbf{j}_{j} \partial \mathbf{j}_{i}}\right|_{\Gamma_{k 0}, \mathbf{j}_{j 0}} \delta \mathbf{j}_{j}, \delta \mathbf{j}_{i}\right\rangle+\left\langle\left.\frac{\partial^{2} \Psi}{\partial \nabla \Gamma_{j} \partial \nabla \Gamma_{i}}\right|_{\Gamma_{k 0}, \mathbf{j}_{j 0}} \delta \nabla \Gamma_{j}, \delta \nabla \Gamma_{i}\right\rangle
\end{aligned}
$$

where we consider the Donkin's theorem (36) in the deduction of (36) for second variation, (for details see Appendix 7.3.). In case when the stationer balance equations are source-free, the terms containing the first variation of the entropy production, satisfying the balance equations and the homogeneous boundary conditions, have zero values. The second terms are positive, because the dissipation potentials in generalized Onsager constitutive theory are strictly convex functions of fluxes and forces, so their functionals, which are generated from the bilinear forms of their Hessian matrices, are positive definite as well. Consequently, the global entropy production in case of homogeneous transport equations is minimal (to form an idea of the role and the significance of the above variational principles see Appendix 7.1.).

\subsection{Examples}

\subsubsection{Deduction of the equation of steady-state heat conduction in anisotropic media. Onsager's problem [3]}

In this part we study the stationer heat conduction in anisotropic solid, e.g. in triclinic crystal. We suppose certain energy deposit $\sigma_{u}$ in all the volume elements of the system, and let the heat conduction tensor be temperature dependent. The stationer balance equation is

$$
\nabla \cdot \mathbf{j}_{u}=\sigma_{u}
$$

where $\mathbf{j}_{u}$ is the internal energy (heat) flux. From this, by multiplication with $1 / T$ we obtain the entropy balance

$$
\nabla \cdot \frac{\mathbf{j}_{u}}{T}=\mathbf{j}_{u} \cdot \nabla\left(\frac{1}{T}\right)+\frac{\sigma_{u}}{T}
$$

where the $\frac{\sigma_{u}}{T}$ is the source generated (e.g. originated from the outer radiation field) entropy production, while the spontaneous entropy production is

$$
\sigma=\mathbf{j}_{u} \cdot \nabla\left(\frac{1}{T}\right)
$$


Supposing quasi-linear constitutive equation, so the heat flux and the $\nabla\left(\frac{1}{T}\right)$ thermodynamic force are connected by the constitutive equation

$$
\mathbf{j}_{u}=\Lambda(T) \nabla\left(\frac{1}{T}\right)
$$

The second order symmetric tensor $\Lambda(T)$ is the specific heat conduction tensor. Its inverse tensor is the specific resistance tensor: $\mathbf{R}(T)=[\boldsymbol{\Lambda}(T)]^{-1}$. Substitute this with the entropy production (115), then we get

$$
\begin{aligned}
& \sigma=\mathbf{j}_{u} \cdot \nabla\left(-\frac{1}{T}\right)=\left[\boldsymbol{\Lambda}(T) \nabla\left(\frac{1}{T}\right)\right] \cdot \nabla\left(\frac{1}{T}\right)= \\
& =\left[\mathbf{R}(T) \mathbf{j}_{u}\right] \cdot \mathbf{j}_{u} \geq 0
\end{aligned}
$$

From this become the second degree homogenous dissipation potentials:

$$
\Psi=\frac{1}{2}\left[\boldsymbol{\Lambda}(T) \nabla\left(\frac{1}{T}\right)\right] \cdot \nabla\left(\frac{1}{T}\right), \Phi=\frac{1}{2}\left[\mathbf{R}(T) \mathbf{j}_{u}\right] \cdot \mathbf{j}_{u}
$$

Consequently the partially restricted variation-task of (73) in this actual case is

$$
\begin{aligned}
& \int_{V} L\left(\mathbf{j}_{u}, \nabla\left(\frac{1}{T}\right),\left(\frac{1}{T}\right)\right) d V= \\
& =\int_{V} \frac{\sigma_{u}}{T}+\frac{1}{2}\left[\boldsymbol{\Lambda}(T) \nabla\left(\frac{1}{T}\right)\right] \cdot \nabla\left(\frac{1}{T}\right)+\frac{1}{2}\left[\mathbf{R}(T) \mathbf{j}_{u}\right] \cdot \mathbf{j}_{u} d V=\min
\end{aligned}
$$

The necessary condition of the minimum is the zero value of the first variation of the above (119) functional

$$
\begin{aligned}
& \delta \int_{V} L\left(\mathbf{j}_{u}, \nabla\left(\frac{1}{T}\right),\left(\frac{1}{T}\right)\right) d V= \\
& =\int_{V}\left(\sigma_{u}-\nabla \cdot\left[\boldsymbol{\Lambda}(T) \nabla\left(\frac{1}{T}\right)\right]\right) \delta\left(\frac{1}{T}\right) d V+\int_{V}\left[\mathbf{R}(T) \mathbf{j}_{u}\right] \cdot \delta \mathbf{j}_{u} d V=0
\end{aligned}
$$

Due to the variations of the temperature are arbitrary and independent from the variations of the heat flux the first term in above equation is zero, consequently we obtain the stationer transport equation

$$
\sigma_{u}-\nabla \cdot\left[\boldsymbol{\Lambda}(T) \nabla\left(\frac{1}{T}\right)\right]=0
$$


The second term in (120) is zero as well, because of the ortogonality relation, which means in this actual case, that the $\nabla\left(\frac{1}{T}\right)$ thermodynamic force is orthogonal to the variation of the divergence-free heat flux. The free variation-task of the problem is

$$
\begin{aligned}
& \int_{V} L\left(\mathbf{j}_{u}, \nabla\left(\frac{1}{T}\right),\left(\frac{1}{T}\right), \lambda\right) d V= \\
& =\int_{V}\left\{\frac{\sigma_{u}}{T}+\frac{1}{2}\left[\Lambda(T) \nabla\left(\frac{1}{T}\right)\right] \cdot \nabla\left(\frac{1}{T}\right)+\frac{1}{2}\left[\mathbf{R}(T) \mathbf{j}_{u}\right] \cdot \mathbf{j}_{u}+\lambda\left(\nabla \cdot \mathbf{j}_{u}-\sigma_{u}\right)\right\} d V=\min
\end{aligned}
$$

In this case the following Euler-Lagrange equations belong to the extremum of the functional as necessary conditions

$$
\begin{aligned}
& \frac{\partial L}{\partial\left(\frac{1}{T}\right)}-\nabla \cdot \frac{\partial L}{\partial \nabla\left(\frac{1}{T}\right)}=0=\sigma_{u}-\nabla \cdot\left[\boldsymbol{\Lambda}(T) \nabla\left(\frac{1}{T}\right)\right] \\
& \frac{\partial L}{\partial \mathbf{j}_{u}}-\nabla \frac{\partial L}{\partial \nabla \cdot \mathbf{j}_{u}}=0=\mathbf{R}(T) \mathbf{j}_{u}-\nabla \lambda \\
& \frac{\partial L}{\partial \lambda}=0=\nabla \cdot \mathbf{j}_{u}-\sigma_{u}
\end{aligned}
$$

showing that the multiplier is the temperature. It is an interesting data of history of nonequilibrium thermodynamics, that Onsager can solve this task only partially, namely, it can deduce only the constitutive equation (116) (for details see Appendix 7.2.)

\subsubsection{Deduction of the transport equation of steady-state electric conduction in inhomogeneous media}

We mean term non-homogeneity in the chemical composition of the system, consequently the mechanism of the electric conduction is place dependent as well. The non-homogeneity in electric DC circuits could be regarded as compartmentation of homogeneous subparts composing the inhomogeneous system step-by step. Let the temperature of the system be constant and have stationer current conduction as a single transport process. The role of the three space-dependent quantities: electric field-strength $(\mathbf{E})$, electric current density $(\mathbf{j})$, and EMF-producing electric field $\mathbf{E}^{(i)}$ generated by other causes as by a battery. The last one is the thermodynamic reaction force, which has to be considered within the current continuity constraint. We suppose, that all of the above physical quantities are functions of the space coordinates. These quantities are dependent on each other; the differential Ohm-law connects them in the linear Onsager constitutive theory

$$
\mathbf{j}=\gamma\left(\mathbf{E}+\mathbf{E}^{(i)}\right)
$$

where $\gamma$ is the electric conductivity. The field equations of electrodynamics of the system are [19] 


$$
\begin{aligned}
& \nabla \cdot \mathbf{j}=0, \nabla \times\left(\frac{\mathbf{j}}{\gamma}\right)=-\nabla \times \mathbf{E}^{(i)}, \\
& \nabla \times \mathbf{E}=0, \nabla \cdot(\gamma \mathbf{E})=-\nabla \cdot\left(\gamma \mathbf{E}^{(i)}\right)
\end{aligned}
$$

To simplify the problem, assume $\nabla \times \mathbf{E}^{(i)}=0, \nabla \times \mathbf{j}=0$.

The stationer transport equation is the consequence of the differential Ohm-law and the continuity equation of the current

$$
\begin{gathered}
\mathbf{j}=-\gamma \nabla\left(\varphi+\varphi^{(i)}\right), \nabla \cdot \mathbf{j}=0 \rightarrow \\
\rightarrow \nabla \cdot(\gamma \nabla \varphi)=-\nabla \cdot\left(\gamma \nabla \varphi^{(i)}\right)
\end{gathered}
$$

Hence the first two equations are equivalent with the third one, where we used the curl-free property of the field-strength and introduced the electric potentials $\varphi, \varphi^{(i)}$.

Prescribe the coordinate of the current density in direction of outer normal vector on $\Omega_{j}$ part of the boundary $\Omega=\Omega_{\mathbf{j}} \cup \Omega \varphi$ and the electric potential on $\Omega \varphi$ : $j_{n}=j^{*}$ on $\Omega_{\mathbf{j}}, \varphi=\varphi^{*}$ on $\Omega_{\varphi}$. Also we suppose that the $\left(\mathbf{j}_{0}, \varphi_{0}\right)$ vector-scalar ordered pair is the unique solution of the equations (126), which are identical(126), and uniform with the (127) transport equation. The entropy production of the system could be calculated by the Joule-law, [19].

$$
\sigma=\frac{1}{\gamma}|\mathbf{j}|^{2}=\mathbf{j} \cdot\left(\mathbf{E}+\mathbf{E}^{(i)}\right)=-\mathbf{j} \cdot \nabla\left(\varphi+\varphi^{(i)}\right)
$$

From this the thermodynamic forces and fluxes as well as the dissipation functions of the linear Onsager constitutive theory and its OM-function follow

$$
\begin{aligned}
& \Phi=\frac{1}{2 \gamma}|\mathbf{j}|^{2}, \Psi=\frac{\gamma}{2}\left|\nabla\left(\varphi+\varphi^{(i)}\right)\right|^{2}, \\
& o(\nabla \varphi, \mathbf{j}, \varphi)=\Phi+\Psi-\sigma \geq 0
\end{aligned}
$$

where in the second equation the equality is valid, when the

$$
\mathbf{j}=-\gamma \nabla\left(\varphi+\varphi^{(i)}\right)
$$

Ohm-law is valid.

Integrate the OM-function on the volume $V$ of the system, while the entropy production is transformed by the surface integral taking into account the continuity equation of the electric current density. Take into account also the boundary conditions prescribing the values of the electric potential and current densities on the boundaries of the system. Than we get the following form of the OM-functional

$$
\begin{aligned}
& O(\nabla \varphi, \mathbf{j}, \varphi):=\int_{V}[\Phi(\mathbf{j}, \varphi)+\Psi(\nabla \varphi, \varphi)] d V+ \\
& -\int_{\Omega \varphi} j_{n} \varphi^{*} d A-\int_{\Omega_{\mathbf{j}}} j^{*} \varphi d A
\end{aligned}
$$


Let its definition domain be

$$
D_{0}(O)=\left\{\begin{array}{l}
(\mathbf{j}, \varphi) \in \mid \nabla \cdot \mathbf{j}=0, \varphi=\varphi^{*} \text { on } \Omega \varphi \\
j_{n}=j^{*} \text { on } \Omega_{\mathbf{j}}
\end{array}\right\}
$$

Let us consider the admissible electric potential as a $C^{2}(V)$ function, and the admissible current density is $C^{1}(V)$. We prove in the following that the $\left(\mathbf{j}_{0}, \varphi_{0}\right)$ pair is the solution to the transport equations (126) if and only if when it is the extremal point of the(131) functional (131), i.e.

$$
O\left(\nabla \varphi_{0}, \mathbf{j}_{0}, \varphi_{0}\right)<O(\nabla \varphi, \mathbf{j}, \varphi), \forall(\mathbf{j}, \varphi) \in D_{0}(O)
$$

To prove this statement we add and deduct again to the integrand the entropy production in (131) and apply the Gauss's divergence theorem, then we get

$$
\begin{aligned}
& O(\nabla \varphi, \mathbf{j}, \varphi)=\int_{V}[-\varphi \nabla \cdot \mathbf{j}+(\Phi+\Psi-\mathbf{j} \cdot \nabla \varphi)] d V= \\
& =\int_{V}[(\Phi+\Psi-\mathbf{j} \cdot \nabla \varphi)] d V \geq 0
\end{aligned}
$$

where we take into account that the current density satisfies the homogeneous balance equation of (126). The functional (134) is always positive on $D_{0}(O)$, and it has its minimum when the Ohm-law is valid as well, also when the $(\mathbf{j}, \varphi)$ pair of functions is the solution to the transport equations (126). Consequently the solution of the transport equations is the minimum place of $O(\nabla \varphi, \mathbf{j}, \varphi)$. Because the sum of the dissipation potentials is equal to the entropy production, the minimum principle of global entropy production is valid in this case.

We can also proof, that one of the necessary conditions belongs to the minimum of the functional are equivalent with the transport equations (126). To do this, we define the $\left(\mathbf{j}+\delta \mathbf{j}, \varphi_{0}+\delta \varphi\right)$ type vector-scalar ordered pairs as admissible, when the variation of the flux $\delta \mathbf{j}$ satisfies the condition of

$$
\nabla \cdot \delta \mathbf{j}=0
$$

homogeneous stationer balance equation, and it is zero on the boundary surface, while the variations of the electric potential $\delta \varphi$ are arbitrary two times continuously differentiable functions. With these assumptions the first variation of (131) is

$$
\begin{aligned}
& \delta O=\int_{V}\left[\left(-\left.\nabla \cdot \frac{\partial \Psi}{\partial \nabla \varphi}\right|_{\varphi_{0}, \nabla \varphi_{0}}\right) \delta \varphi+\left.\frac{\partial \Phi}{\partial \mathbf{j}}\right|_{\varphi_{0}, \mathbf{j}_{0}} \cdot \delta \mathbf{j}\right] d V= \\
& =\int_{V}\left[\left(-\left.\nabla \cdot \frac{1}{\gamma} \nabla\left(\varphi+\varphi^{(i)}\right)\right|_{\varphi_{0}, \nabla \varphi_{0}}\right) \delta \varphi+\left.\frac{\partial \Phi}{\partial \mathbf{j}}\right|_{\varphi_{0}, \mathbf{j}_{0}} \cdot \delta \mathbf{j}\right] d V=0
\end{aligned}
$$


As since the necessary condition of the extremum of functional is the zero value of its first variation. Due to the variations of the potential are arbitrary and independent of the variations of the flux, than we get with $\delta \varphi=0$

$$
\int_{V}\left[\left.\frac{\partial \Phi}{\partial \mathbf{j}}\right|_{\varphi_{0}, \mathbf{j}_{0}} \cdot \delta \mathbf{j}\right] d V=0
$$

Using this, for arbitrary $\delta \varphi$ it follows from (136):

$$
\delta O=\int_{V}\left(-\left.\nabla \cdot \frac{1}{\gamma} \nabla\left(\varphi+\varphi^{(i)}\right)\right|_{\varphi_{0}, \nabla \varphi_{0}}\right) \delta \varphi d V=0
$$

and from this by the fundamental lemma of variational calculus follows the transport equation (127):

$$
\nabla \cdot \frac{1}{\gamma} \nabla\left(\varphi+\varphi^{(i)}\right)=0
$$

Consequently one of the necessary conditions of the extremum is the transport equation. The second one has to be considered as the functional form used in the discussions above, regarding on $\left.\frac{\partial \Phi}{\partial \mathbf{j}_{i}}\right|_{\Gamma_{k 0}, \mathbf{j}_{j 0}}=-\nabla\left(\varphi+\varphi^{(i)}\right)$, from what the direct proof of orthogonality is obtained

$$
\int_{V}\left(\left.\frac{\partial \Phi}{\partial \mathbf{j}}\right|_{\varphi_{0}, \mathbf{j}_{0}} \cdot \delta \mathbf{j}\right) d V=\int_{V}\left(-\left(\varphi+\varphi^{(i)}\right) \cdot \nabla \cdot \delta \mathbf{j}\right) d V=0
$$

\subsubsection{Murray's law of mammalian circulatory systems}

Murray C.D. suggested [20], that the inner radius of blood vessel increases as an outcome of a compromises between the advantage of increasing of the lumen, (which decreases the viscous dissipation of blood flow) and the disadvantage of increasing blood volume, (which increases the metabolic demand to maintain the physiological quality of blood) [21]. For example, because the red blood cells must be produced continuously to replace vanishing cells. The Murray's "cost function" is the total power required to maintain the blood flow and the prescribed stationer quality of blood [19], [20]. According to the first law of thermodynamics which is valid on the stationer cases, the total demand of power is identical with the sum of the viscous dissipation of the stationer flow of blood, and the energy dissipation of the irreversible chemical, physical, biological changes in blood. Due to these, the "cost function" as the energy-dissipation will be discussed in the next part, and use the following entropy production. Let the thermodynamic system be a part of vessel with length $L$, radius $r$ and has constant $T$ temperature. Consider the flow stationer, neglecting the changes by the living pulsation of the blood. The blood is not compressible, and we do not consider the dilatation of the vessels. In this case the homogeneous balances and the minimum principle of global entropy production are valid. Let us study the constitutive equations of the system, when the blood is Newtonian liquid, and the pressure difference is 
$\Delta p$, the volume-current of the vessel is $I$. Their connection is described by the HagenPoiseuille-law

$$
\Delta p=\frac{8 \eta L}{\pi r^{4}} I
$$

from where the viscous energy dissipation per unit time is

$$
\Delta p I=\frac{8 \eta L}{\pi r^{4}} I^{2}
$$

We assume that power of the irreversible processes which change the blood quality are proportional with its volume

$$
P_{\text {deg }}=k r^{2} \pi L
$$

where $k$ is the specific energy dissipation per unit time. Consequently, the global entropy production is

$$
P=\frac{\frac{8 \eta L}{\pi r^{4}} I^{2}+k r^{2} \pi L}{T}
$$

It could be shown that the entropy production is a strictly convex function of the radius of the vessel. The necessary condition of the minimum gives the Murray-law

$$
\frac{d P}{d r}=\frac{d}{d r} \frac{\frac{8 \eta L}{\pi r^{4}} I^{2}+k r^{2} \pi L}{T}=0 \rightarrow I=\frac{\pi}{4}\left(\frac{k}{\eta}\right)^{\frac{1}{2}} r^{3}
$$

This law expresses that the volume current of blood is proportional with the third power of the radius of the vessel when the entropy production is minimal.

Let us study now the areas of bifurcations. One of the characteristic properties of vasculature is that it divides into smaller and smaller vessels down to the level of capillaries. These divisions are the bifurcations. When the mother-vessel with radius $r_{0}$ divides into two daughter vessels with $r_{1}$ and $r_{2}$ radii we can introduce the ratios

$$
\alpha=\frac{r_{2}}{r_{1}}, \beta=\frac{r_{1}^{2}+r_{2}^{2}}{r_{0}^{2}}
$$

characterizing the bifurcation. The volume currents fulfill the Kirchhoff's first law

$$
I_{0}=I_{1}+I_{2}
$$

From this and from the Murray's law for the above introduced ratios follow

$$
\frac{r_{1}}{r_{0}}=\frac{1}{\left(1+\alpha^{3}\right)^{\frac{1}{3}}}, \frac{r_{2}}{r_{0}}=\frac{\alpha}{\left(1+\alpha^{3}\right)^{\frac{1}{3}}}, \beta=\frac{1+\alpha^{2}}{\left(1+\alpha^{3}\right)^{\frac{2}{3}}}
$$


When the bifurcation is symmetric (also $\alpha=1$ ), then

$$
\frac{r_{1}}{r_{0}}=\frac{r_{2}}{r_{0}}=\frac{\alpha}{\left(1+\alpha^{3}\right)^{\frac{1}{3}}} \cong 0.8, \beta=\frac{1+\alpha^{2}}{\left(1+\alpha^{3}\right)^{\frac{2}{3}}} \cong 1,26
$$

This clearly shows that the vessel's cross section increases and the average velocity of the blood decreases by $26 \%$ in symmetric bifurcation following the Murray's law.

Substituting the Murray's law into the entropy production (144), we get the global entropy production in optimal case for the vessel

$$
P=\frac{\frac{8 \eta L}{\pi r^{4}} I^{2}+k r^{2} \pi L}{T}=\frac{1}{T} \frac{3}{2} k r^{2} \pi L
$$

The minimal entropy production has general consequences on the geometry of the vascular network. The most important one that the supply-network must not have loops, otherwise we could neglect this hypothetic loop without changing the supply with blood in any of the junction points. Also, we could decrease the entropy production without the missing supply of the junctions. The network, which has no loops called tree. So the minimal entropy production guarantees the tree-character of the vascular geometry.

The other important characteristic of bifurcation pattern is the bifurcation angle (see Fig. 1.a.), which also satisfies the minimal entropy production principle. To prove this consider the junction geometry determined by the $A, B$, and $C$ points in Fig. 1.b. Its entropy production is

$$
P=\frac{1}{T} \frac{3}{2} \pi k\left(r_{0}^{2} L_{0}+r_{1}^{2} L_{1}+r_{2}^{2} \pi L_{2}\right)
$$

When we move the junction point $E$, the length of the sections will be varied as well, which changes the entropy production too. This problem could be elucidated by a mechanical analogue, which helps to show that the function (151) has a minimum. Draw the junction of Fig. 2.a. on a simple sheet of paper, and punch it at the points $A, B$ and $C$. Let we load three strings with equal length with weights $m_{A}=\frac{1}{T} \frac{3}{2} \frac{\pi k}{g} r_{0}^{2}, m_{B}=\frac{1}{T} \frac{3}{2} \frac{\pi k}{g} r_{1}^{2}$ and $m_{C}=\frac{1}{T} \frac{3}{2} \frac{\pi k}{g} r_{2}^{2}$. Reeve the strings on corresponding $A, B$ and $C$ punches, and lift up the free ends of the strings by $L_{0}, L_{1}, L_{2}$ lengths at the $m_{A}, m_{B}$, and $m_{C}$ weights, respectively. Knot the ends of the strings in one. The value of the potential energy of this system will be equal with the value of the entropy production of (151). Leaving the knot free, and neglect the friction, due to the Dirichlet principle the system will occupy its equilibrium state which has minimal potential energy. The geometry of the strings on the sheet will produce the optimal junction geometry. Due to this, the equilibrium is the resultant of the forces in the knot is zero (also the forces form a triangle; see Fig. 2.b). Following the analogy, from this the junction angles belonging to the minimum of global entropy production can be calculated by the law of cosines 


$$
\cos \Theta_{1}=\frac{r_{0}^{4}+r_{1}^{4}-r_{2}^{4}}{2 r_{0}^{2} r_{1}^{2}}, \cos \Theta_{2}=\frac{r_{0}^{4}+r_{2}^{4}-r_{1}^{4}}{2 r_{0}^{2} r_{1}^{2}}
$$

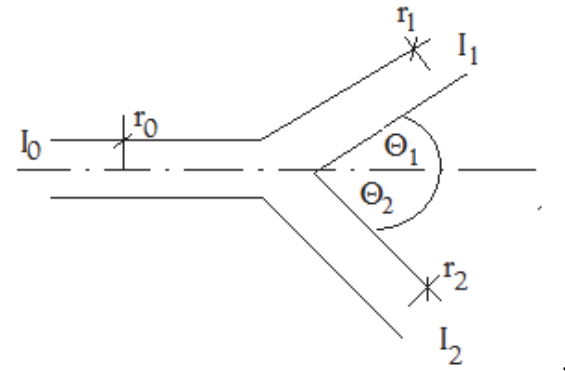

(a)

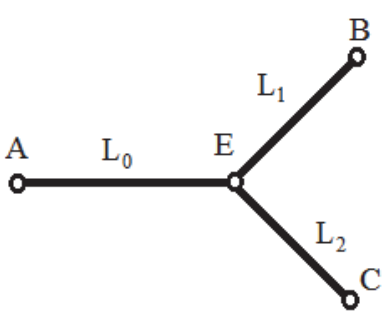

(b)

Fig. 1. Sketch for determination of the bifurcation angle.

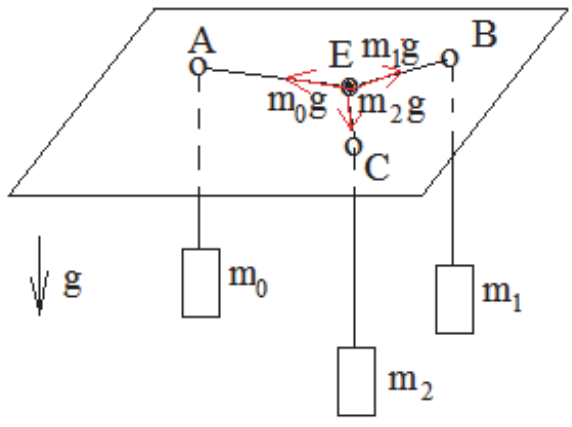

(a)

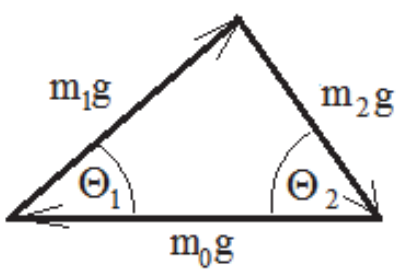

(b)

Fig. 2. Mechanical analogue for determination of bifurcation angle.

According to the experiments the Murray's law is not always accurate enough to describe the bifurcation properties of the vascular network. Recently Huo and Kassab [22] offered a correction of the (143) constitutive equation, based on the allometric scaling law with $3 / 4$ power [23]. The essence of their proposal is that they regard the cumulative vessel as thermodynamic system. The $V$ volume of this cumulative vessel by the allometric scaling depends on the radius by $V=k_{v} \pi r^{\frac{2}{3}} L$, where $k_{v}$ is constant. Hence the constitutive law (143) will be

$$
P_{\operatorname{deg} r}=k k_{v} \pi r^{\frac{2}{3}} L
$$


and the entropy production has a form

$$
P=\frac{\frac{8 \eta L}{\pi r^{4}} I^{2}+k k_{v} r^{\frac{2}{3}} \pi L}{T}
$$

\subsection{Exercises}

2.3. 1. Show that the Euler-Lagrange equations are identical with the inhomogeneous transport equations, when the variation principle (73) is a free-task varying only the intensive parameters. (Such type of non-exact principles of thermodynamics are called the partial variational principles [24].

2.3. 2. Show that in the case of inhomogeneous steady-state balance equations the fluctuation of GEP is not zero in the first order.

2.3. 3. Show with the help of the short calculus that fluctuation of the sum of the global spontaneous entropy production $\left\langle\nabla \Gamma_{i}, \mathbf{j}_{i}\right\rangle$ and the entropy production $\left\langle\sigma_{i}, \Gamma_{i}\right\rangle$ generated by the sources is zero in the first order in a steady-state system.

2.3. 4. Show that from the minimum principle of the global entropy production (which as we seen, is a restricted variation principle) can be constructed by Lagrange's method of multipliers in the following free variational-task:

$$
\begin{gathered}
\int_{V} L\left(\mathbf{j}_{j}, \nabla \mathbf{j}_{j}, \nabla \Gamma_{j}, \Gamma_{k}, \lambda_{i}\right) d V-\int_{\Omega_{\Gamma_{j}}} j_{j n} \Gamma_{j}^{*} d A-\int_{\Omega_{j_{j}}} j_{j}^{*} \Gamma_{j} d A=\min \\
L\left(\mathbf{j}_{j}, \nabla \mathbf{j}_{j}, \nabla \Gamma_{j}, \Gamma_{k}, \lambda_{i}\right)=\Phi\left(\mathbf{j}_{j}, \Gamma_{k}\right)+\Psi\left(\nabla \Gamma_{j}, \Gamma_{k}\right)+\lambda_{i} \nabla \cdot \mathbf{j}_{i}
\end{gathered}
$$

2.3. 5. Show with short calculus, that in the chapter of 2.2.2. the first variation of the global entropy production is zero, the second one is positive definite.

2.3. 6. Make a free variation-task from the partially restricted variation (131) by Lagrange's method of multipliers. Give the Euler-Lagrange equations.

2.3. 7. Determine the law corresponding with Murray's one when the global entropy production of the vessel has a form of (154).

2.3. 8. Determine the bifurcation angles, when the global entropy production of vessel has a form of (154).

\section{Deduction of a class of constitutive equations from the principle of global entropy production}

Up to this section we have used the minimum principle of the steady-state system and the connected minimum principle of the global entropy production to deduce the transport equations. In the following we shall give an equivalent functional formulation of the minimum principle of the global entropy production. We don't assume the validity of generalized Onsager constitutive theory and that the dissipation potentials are homogeneous Euler's functions. As opposed to this, we shall investigate the restrictions by minimum principle of global entropy production in the possible mathematical form of the constitutive equations.

We introduce from the minimum principle of global entropy production 


$$
P=\int_{V} L\left(X_{j}, j_{j}\right) d V=\int_{V} X_{j} j_{j} d V=\min .
$$

It is well known from the variational calculus that the mathematical character and the necessary condition of a extremum are invariant to the transformations of the Langrangian

$$
L\left(X_{j}, j_{j}\right) \rightarrow L\left(X_{j}, j_{j}\right)+\nabla \cdot \mathbf{F}\left(X_{j}, j_{j}\right)
$$

where $\mathbf{F}$ is an arbitrary vector function with respect to $\Gamma$ 's, $X$ 's and $j$ 's.

Assume that the generator function $\mathbf{F}$ has the following bilinear form

$$
\mathbf{F}\left(X_{j}, j_{j}\right):=-\mu \mathbf{J}_{\mathbf{s}}=-\mu \Gamma_{i} \mathbf{j}_{i},
$$

where $\mu$ is an arbitrary positive scalar. With this, the variation principle will have the following form

$$
\begin{aligned}
& S=\int_{V} L\left(X_{j}, j_{j}\right) d V=\min , \\
& L\left(X_{j}, j_{j}\right)=-(\lambda+1) \Gamma_{j} \nabla \cdot \mathbf{j}_{j}-\lambda \nabla \Gamma_{j} \mathbf{j}_{j}
\end{aligned}
$$

where we take into account that in the case of irreversible vector processes the $\nabla \Gamma_{j}$ are the conjugated thermodynamic force to the $\mathbf{j}_{j}$ flux, and we introduced the $\lambda:=\mu-1$ notation. To determine the possible form of the constitutive equations deduced from the above variation principle, starting with the thermodynamic equilibrium. This could be regarded as stationer state, where the global entropy production has its absolute minimum. As we know from irreversible thermodynamics this is zero and the forces and currents are independent and also have zero values.

Supposing there are missing connections between the forces and currents, the variational principle (159) is the necessary condition for the extremum at the free variation of the intensives and fluxes we get the Euler-Lagrange equations belonging to the variation principle (159) are the following

$$
\nabla \cdot \mathbf{j}_{j}=0, \quad \nabla \Gamma_{j}=0,(j=1,2, \ldots, N) .
$$

which are compatible with thermodynamic equilibrium of the system. Supposing the constitutive equations between the forces and fluxes as

$$
\begin{aligned}
& \nabla \Gamma_{i}=\mathbf{f}_{i}\left(\mathbf{j}_{j}, \Gamma_{k}\right),(i, j=1,2, \ldots, N), \\
& \frac{\partial \Gamma_{i}}{\partial x_{u}}=f_{i u}\left(j_{j u}, \Gamma_{k}\right),(i, j, k=1,2, \ldots, N),(u=1,2,3) .
\end{aligned}
$$

and their exact mathematical form is unknown. We study how the Euler-Lagrange equations belonging to the variation principle (159) limit the possible mathematical form of the constitutive equations.

Substituting the constitutive equations (161) into the Lagrangian of (159), then we get the following Lagrangian 


$$
L=-(\lambda+1) \Gamma_{i} \frac{\partial j_{i u}}{\partial x_{u}}-\lambda f_{i v} j_{i v},(i=1,2, \ldots, N),(u, v=1,2,3)
$$

In consequence, the necessary condition of the extremum gives the Euler-Lagrange equations

$$
\begin{aligned}
& \frac{\partial L}{\partial \Gamma_{i}}=0-(\lambda+1) \frac{\partial j_{i u}}{\partial x_{u}}-\lambda \frac{\partial f_{i u} j_{i u}}{\partial \Gamma_{i}}=0, \\
& \frac{\partial L}{\partial f_{i u}}=0-(\lambda+1) \frac{\partial \Gamma_{i}}{\partial x_{u}}-\lambda f_{i u}-\lambda j_{i v} \frac{\partial f_{i v}}{\partial j_{i u}}= \\
& =f_{i u}-\lambda j_{i v} \frac{\partial f_{i v}}{\partial j_{i u}}=0,(i=1,2, \ldots, N),(u, v=1,2,3)
\end{aligned}
$$

The Euler-Lagrange equations belonging to the variation of the thermodynamic fluxes are

$$
f_{i u}=\lambda j_{i v} \frac{\partial f_{i v}}{\partial j_{i u}}(i=1,2, \ldots, N),(u, v=1,2,3)
$$

Assume that $f_{i u}$ 's are two times continuously differentiable functions of the fluxes, and then the following derivatives are

$$
\begin{aligned}
& \frac{\partial f_{i u}}{\partial j_{k v}}=\lambda \frac{\partial f_{k v}}{\partial j_{i u}}+\lambda j_{k v} \frac{\partial^{2} f_{k v}}{\partial j_{k v} \partial j_{i u}}, \\
& \frac{\partial f_{k v}}{\partial j_{i u}}=\lambda \frac{\partial f_{i u}}{\partial j_{k v}}+\lambda j_{i u} \frac{\partial^{2} f_{i u}}{\partial j_{i u} \partial j_{k v}},(i=1,2, \ldots, N),(u, v=1,2,3)
\end{aligned}
$$

From the equations (164) and the Young' theorem of the continuous second partial derivatives, we get

$$
\frac{\partial f_{i u}}{\partial j_{k v}}-\frac{\partial f_{k v}}{\partial j_{i u}}=\lambda\left(\frac{\partial f_{k v}}{\partial j_{i u}}-\frac{\partial f_{i u}}{\partial j_{k v}}\right),(i=1,2, \ldots, N),(u, v=1,2,3)
$$

Since these equations are obtained for every $\lambda$, we conclude the generalized reciprocal relations

$$
\frac{\partial f_{i u}}{\partial j_{k v}}=\frac{\partial f_{k v}}{\partial j_{i u}},(i=1,2, \ldots, N),(u, v=1,2,3)
$$

This very important result states that the necessary condition of the minimum principle of the global entropy production is that the constitutive equations must belong to the generalized Onsager constitutive theory. This property implies the existence of the dissipation potentials, which are constructed by Legendre transform to each other. Furthermore, the Donkin's theorem of entropy production (42) is valid, so the derivatives of the entropy production by the intensive parameters vanish. Consequently, the first group of the Euler-Lagrange equations (163) reduced to the homogeneous steady-state balance equations: 


$$
\frac{\partial j_{i u}}{\partial x_{u}}=0,(i=1,2, \ldots, N),(u=1,2,3)
$$

which are necessary to the minimum of GEP as we showed above. Consequently, the proposed variation principle is equivalent with a class of stationer transport equations, where the constitutive equations are generalized Onsager-like. There are more constitutive properties resulting from this aspect of minimum entropy production. Studying these, substitute the constitutive equations into the entropy production

$$
\sigma^{*}=f_{i v} j_{i v},(i=1,2, \ldots, N),(u=1,2,3)
$$

and derive it with respect to the fluxes

$$
\frac{\partial \sigma^{*}}{\partial j_{i v}}=f_{i v}+j_{i u} \frac{\partial f_{i u}}{\partial j_{i v}},(i=1,2, \ldots, N),(u=1,2,3)
$$

From these equations using the equation (164), we get

$$
\frac{\partial \sigma^{*}}{\partial j_{i v}}=\frac{\lambda+1}{\lambda} f_{i v},(i=1,2, \ldots, N),(u=1,2,3)
$$

and from this we obtain

$$
j_{i v} \frac{\partial \sigma^{*}}{\partial j_{i v}}=\frac{\lambda+1}{\lambda} j_{i v} f_{i v}=\frac{\lambda+1}{\lambda} \sigma^{*}
$$

So the entropy production is homogeneous Euler's function of degree $\frac{\lambda+1}{\lambda}$ with respect to the fluxes. From this property of entropy production and from the physical meaning of flux potential described in (26), we get the connection between the flux dissipation potential and entropy production, like

$$
\begin{aligned}
& \Phi\left(j_{l}, \Gamma_{k}\right)=\int_{0}^{\infty} \sigma^{*}\left(e^{-t} j_{j}, \Gamma_{k}\right) d t=\sigma^{*}\left(j_{j}, \Gamma_{k}\right) \int_{0}^{\infty} e^{-t \frac{\lambda+1}{\lambda}} d t= \\
& =\frac{\lambda}{\lambda+1} \sigma^{*}\left(j_{j}, \Gamma_{k}\right)
\end{aligned}
$$

Hence the flux potential is also a homogeneous Euler's function of degree $\frac{\lambda+1}{\lambda}$, and it is a $\frac{\lambda}{\lambda+1}$-th part of the entropy production. Since the force dissipation potential is the Legrende transform of the flux potential, so it follows that it (44) is homogeneous Euler's function of degree $\lambda+1$ of the forces and $\frac{1}{\lambda+1}$-th part of the entropy production. When $\lambda=1$ then the dissipation potentials are the same as it is predicted in the linear Onsager's theory. 
In summary, the previously given form of the minimal principle of entropy production leads to a class of generalized Onsager constitutive theory, which is also direct generalization of the linear Onsager's theory having their dissipation potentials as homogeneous Euler's functions.

\subsection{Examples}

\subsubsection{Thermo-diffusion in triclinic system}

In case of thermo-diffusion the global entropy production [25], [1], [2] is

$$
\begin{aligned}
& P=\int_{V}\left[\mathbf{j}_{q} \cdot \nabla\left(\frac{1}{T}\right)-\mathbf{j}_{\alpha} \cdot \nabla\left(\frac{\mu_{\alpha}}{T}\right)\right] d V= \\
& =\int_{V}\left[j_{q i} \frac{\partial}{\partial x_{i}}\left(\frac{1}{T}\right)-j_{\alpha i} \frac{\partial}{\partial x_{i}}\left(\frac{\mu_{\alpha}}{T}\right)\right] d V,(\alpha=1,2,3, \ldots, K),(i=1,2,3)
\end{aligned}
$$

where $\mathbf{j}_{q}$ is the heat-current density vector, $\mathbf{j}_{\alpha}$ is the mass-current density of the $\alpha$-th component, $T$ is the temperature and $\mu_{\alpha}$ is the chemical potential of the $\alpha$-th component. Let us consider the following linear constitutive equations

$$
\begin{aligned}
& \frac{\partial}{\partial x_{i}}\left(\frac{1}{T}\right)=R_{i k} j_{q k}-\mathrm{K}_{i k}^{(\alpha)} j_{\alpha k} \\
& \frac{\partial}{\partial x_{i}}\left(\frac{\mu_{\alpha}}{T}\right)=-D_{i k}^{(\alpha \beta)} j_{\beta k}-D_{i k}^{(\alpha)} j_{q k}
\end{aligned}
$$

where $\left[R_{i k}\right]$ is the specific heat-resistivity tensor, $\left[\mathrm{K}_{i k}^{(\alpha)}\right]$ 's are second order tensors connect the temperature gradient and the fluxes of chemical components, $\left[D_{i k}^{(\alpha \beta)}\right]$ is the diffusion tensor, and $\left[D_{i k}^{(\alpha)}\right]$ 's are second order tensors connect the chemical potential gradients and the heat-flux.

With these equations the Lagrangian of the problem is (162) :

$$
\begin{aligned}
& L=-\frac{\lambda+1}{T} \frac{\partial j_{q i}}{\partial x_{i}}-(\lambda+1) \frac{\mu_{\alpha}}{T} \frac{\partial j_{\alpha i}}{\partial x_{i}}-\lambda\left(R_{i k} j_{q i} j_{q k}-\mathrm{K}_{i k}^{(\alpha)} j_{q i} j_{\alpha k}\right. \\
& \left.-D_{i k}^{(\alpha)} j_{q i} j_{\alpha k}-D_{i k}^{(\alpha \beta)} j_{\alpha i} j_{\beta k}\right)
\end{aligned}
$$

It is possible to determine the relations among the kinetic coefficients from the EulerLagrange- equations belonging to the variation of i-th coordinate $j_{q i}$ and $j_{\alpha i}$ of the heat and a-th chemical component current densities

$$
\begin{aligned}
& j_{q i}\left(\lambda R_{i k}-R_{k i}\right)+j_{\alpha i}\left(\lambda \mathrm{K}_{i k}^{(\alpha)}-D_{k i}^{(\alpha)}\right)=0 \\
& j_{q i}\left(\lambda \mathrm{K}_{k i}^{(\alpha)}-D_{i k}^{(\alpha)}\right)+j_{\alpha i}\left(\lambda D_{i k}^{(\alpha \beta)}-D_{k i}^{(\beta \alpha)}\right)=0
\end{aligned}
$$

From these (due to the arbitrary current densities) we receive 


$$
\begin{aligned}
& j_{q i}\left(\lambda R_{i k}-R_{k i}\right)=0 \\
& j_{\alpha i}\left(\lambda \mathrm{K}_{k i}^{(\alpha)}-D_{i k}^{(\alpha)}\right)=0 \\
& j_{\alpha i}\left(\lambda D_{i k}^{(\alpha \beta)}-D_{k i}^{(\beta \alpha)}\right)=0
\end{aligned}
$$

Since in the case of linear constitutive equations the $\lambda=1$, and hence we get the symmetry relations as follows

$$
R_{i k}=R_{i k}, \quad \mathrm{~K}_{k i}^{(\alpha)}=D_{i k}^{\alpha}, \quad D_{i k}^{(\alpha \beta)}=D_{k i}^{(\beta \alpha)}
$$

With the application of the Curie principle from this general result we have the reciprocal relations for isotropic case, [1], [2], [25]. Due to the Curie-principle the $\left[R_{i k}\right],\left[\mathrm{K}_{i k}^{(\alpha)}\right]$, $\left[D_{i k}^{(\alpha)}\right]$, and $\left[D_{i k}^{(\alpha \beta)}\right]$ tensors are simplified in isotropic case in the form of

$$
R_{i k}=R \delta_{i k}, \quad \mathrm{~K}_{i k}^{(\alpha)}=K^{(\alpha)} \delta_{i k}, D_{i k}^{\alpha}=D^{(\alpha)} \delta_{i k}, D_{i k}^{(\alpha \beta)}=D^{(\alpha \beta)} \delta_{i k}
$$

where $\delta_{i k}$ is a Kronecker-delta. From this we receive the well-known [26] reciprocal relations

$$
K^{(\alpha)}=D^{(\alpha)}, D^{(\alpha \beta)}=D^{(\beta \alpha)},(\alpha, \beta=1,2,3, \ldots, K)
$$

\subsubsection{Electric conductivity in triclinic system in presence of external magnetic field}

For simplicity we suppose the investigated process is isotherm. In this case the entropy production can be written as [1], [2]:

$$
\sigma=\frac{1}{T} \mathbf{j} \cdot \mathbf{E}=-\frac{1}{T} \mathbf{j} \cdot \nabla \varphi
$$

where $\mathbf{j}$ is the electric current density, and the intensive parameter $\varphi$ is the electric potential. Let the constitutive equation be quasi-linear

$$
\nabla \varphi=-\mathbf{R} \mathbf{j}-\kappa \mathbf{j} \times \mathbf{B}:=\mathbf{R}(\mathbf{B}) \mathbf{j}, \quad \frac{\partial \varphi}{\partial x_{i}}=-R_{i j} j_{j}-\kappa \varepsilon_{i j k} j_{j} B_{k}
$$

where $\mathbf{R}$ is the specific resistivity tensor of the given crystal, $\mathrm{k}$ is a constant, characterizing of the material, and $\varepsilon_{i j k}$ is a permutation symbol, equals 1 when the series of $i, j, k$ is even or 1 when odd permutation of numbers 1,2,3. The corresponding Lagrangian is

$$
L=-(\lambda+1) \varphi \frac{\partial j_{i}}{\partial x_{i}}+\lambda R_{i j} j_{i} j_{j}+\lambda \kappa \varepsilon_{i j k} j_{i} j_{j} B_{k}
$$

With variation of $j$-th coordinate $j j$ of electric current density we get the following EulerLagrange-equations

$$
\begin{aligned}
& \lambda\left(R_{i j}+R_{j i}\right) j_{i}+\lambda \kappa\left(\varepsilon_{i j k} j_{i} B_{k}+\varepsilon_{j i k} j_{i} B_{k}\right)= \\
& =(\lambda+1)\left(R_{i j} j_{i}+\varepsilon_{i j k} j_{i} B_{k}\right)
\end{aligned}
$$


which by algebraic rearrangement leads to the equations

$$
\lambda R_{j i} j_{i}+\lambda \kappa \varepsilon_{j i k} j_{i} B_{k}=R_{i j} j_{i}+\varepsilon_{i j k} j_{i} B_{k}
$$

Regarding the $\lambda=1$ in case of linear constitutive equations, we receive the relations

$$
R_{j i} j_{i}+\kappa \varepsilon_{j i k} j_{i} B_{k}=R_{i j} j_{i}+\kappa \varepsilon_{i j k} j_{i} B_{k}
$$

which is valid for every $j_{j}$ and $B_{k}$. However, it could be satisfied in case only when the elements of the resistivity tensor are as follows

$$
R_{i j}(\mathbf{B})=R_{i j}+\kappa \varepsilon_{i j k} B_{k},(i, j, k=1,2,3),
$$

and $R_{i j}(\mathbf{B})$ satisfy the well-known

$$
R_{i j}(\mathbf{B})=R_{j i}(-\mathbf{B}),(i, j, k=1,2,3),
$$

reciprocity relations.

\section{Evolution toward steady-state. Glansdorff-Prigogine general evolution criterion. The Glansdorff - Prigogine theorem of the minimum entropy production}

The above parts show the minimum principle for vector processes in the frame of the generalized Onsager constitutive theory by the directions of Onsager's last dissipation of energy principle. We had seen above that in case of source-free balances, this principle is equivalent with the principle of minimal entropy production. The equivalence of the two theorems in the frame of the linear constitutive theory was proven by Gyarmati [2] first. Furthermore, we showed that in case when the principle of minimal entropy production is used for the determination of the possible forms of constitutive equations, the results are similar to the linear theory in the frame of the Onsager's constitutive theory, where the dissipation potentials are homogeneous Euler's functions.

Glansdorff and Prigogine had chosen different approach for the minimum entropy production in the frame of linear constitutive theory $\left.{ }^{27}\right],[28]$. In the following we shall fit this theory into the generalized Onsager constitutive theory.

The original Glansdorff-Prigogine theorem of the minimum global entropy production may be described as follows. In every system satisfying the reciprocity relations of the linear Onsager's irreversible thermodynamics, where the convective velocity is zero and where the local value of the intensive parameters $\Gamma_{k},(k=1,2, \ldots, N)$ at the boundary of the system are fixed, then the stationary state corresponds to a minimum of the global entropy production of the system. During the evolution of the system from its initial state to its stationary state, the rate of the global entropy production (RGEP)

$$
\frac{d P}{d t}=\frac{d}{d t} \int_{V} \sigma d V=\int_{V} \frac{\partial X_{j}}{\partial t} j_{j} d V+\int_{V} X_{j} \frac{\partial j_{j}}{\partial t} d V<0 .
$$

decreases monotony and at the stationary state 


$$
\frac{d P}{d t}=0
$$

It can be shown that these properties of the global entropy production (GEP) guarantees the stability of non-equilibrium stationer state.

If the assumptions of the linear Onsager constitutive theory are not fulfilled, during the evolution of the system a more general theorem can still be derived in non-stationer state, namely,

$$
\frac{d P_{X}}{d t}=\int_{V} \frac{\partial X_{j}}{\partial t} j_{j} d V<0 .
$$

and

$$
\frac{d P_{X}}{d t}=0
$$

at a stationer state. This theorem is the Glansdorff-Prigogine general evolution criterion which is also a universal necessary condition to the existence of steady state [27], [28]. Consequently, the range of this theorem is independent of the assumption that the constitutive relations are linear, also it is valid in case of the generalized Onsager constitutive theory. In the followings we assume that the thermodynamic forces $X_{l}$ and fluxes $j_{l}$ belong to generalized Onsager constitutive theory. In this case, as a consequence of the (31) Legendre transformation the global entropy $P$ produced inside the system can be formulated with the dissipation potentials, i.e.

$$
P=\int_{V} \sigma d V=\int_{V} X_{j} j_{j} d V=\int_{V}\left[\Psi\left(X_{l}, \Gamma_{k}\right)+\Phi\left(j_{l}, \Gamma_{k}\right)\right] d V,
$$

while the rate of the GEP can be written as

$$
\frac{d P}{d t}=\int_{V} \frac{\partial X_{j}}{\partial t} \frac{\partial \Psi}{\partial X_{j}} d V+\int_{V} \frac{\partial j_{j}}{\partial t} \frac{\partial \Phi}{\partial j_{j}} d V,
$$

where $V$ is the volume of the system, which is at rest in the frame of reference.

The Glansdorff-Prigogine general evolution criterion, in case of time independent boundary conditions, which are valid during the evolution of the thermodynamic steady state, can be formulated as

$$
\frac{d P_{X}}{d t}=\int_{V} \frac{\partial X_{j}}{\partial t} \frac{\partial \Psi}{\partial X_{j}} d V \leq 0
$$

No similar conditions exist for RGEP and the flux part of RGEP in generalized Onsager constitutive theory. Also, the assumptions of this theory, contrary to the linear constitutive theory, do not guarantee the stabile extremum of the GEP at a thermodynamic steady-state. It is well known that in the linear constitutive theory the dissipation potentials are second order homogeneous Euler's functions. This is a central property of the potentials, which guarantees that the two parts of the GEP are proportional to the GEP and are equal with 
each other. Consequently, if we assume that the dissipation potentials are arbitrary homogeneous Euler' functions in generalized Onsager constitutive theory, then the GEP have similar properties. Consider the force potential as Euler's function of degree $k$ with respect to the $X^{\prime}$ s, the GEP in this case from (43) is

$$
P=\int_{V} \sigma d V=\int_{V} X_{j} j_{j} d V=\int_{V} X_{j} \frac{\partial \Psi}{X_{j}} d V=k \int_{V} \Psi d V
$$

and the RGEP is

$$
\frac{d}{d t} P=k \int_{V} \frac{\partial \Psi}{\partial X_{j}} \frac{\partial X_{j}}{\partial t} d V
$$

According to the Glansdorff-Prigogine general evolutions criterion (192), from the above equation we get the necessary conditions of the minimum of the entropy production at the thermodynamics steady state

$$
\frac{d}{d t} P=k \int_{V} \frac{\partial \Psi}{\partial X_{j}} \frac{\partial X_{j}}{\partial t} d V<0 \text { away fromsteady state }
$$

and

$$
\frac{d}{d t} P=0 \text { atsteady state }
$$

Also the Glansdorff-Prigogine principle of the minimum of the global entropy production is valid in the generalized Onsager constitutive theory, when the dissipation functions are homogeneous Euler's functions with respect to thermodynamic forces and fluxes. It is evident, that the above criteria fulfilled for RGEP are sufficient for a stabile stationer state and it is not necessary. So the stationer state can exist not meeting this criteria.

It is important to note that the Glansdorff-Prigogine principle of the minimum of the global entropy production is more general than our above formulated minimum principle for vector processes even in the class of constitutive equations where the dissipation potentials are homogeneous Euler's functions. The reason of the limited sphere of operation that the general evolution criteria in (192) is valid for chemical reactions [29], [1], [2] which are crucial for the biological applications. The present generalization however is important, because any dissipation potential and the entropy production could be used as kinetic potential, which are important in the studies of the stability of chemical reactions [28], [29].

\subsection{Stability of steady-state}

The Glansdorff-Prigogine general evolution criterion involves the minimum of global entropy production in such a constitutive theory where the potentials are homogeneous Euler's functions. We show below the strictly convex property of dissipation potentials guarantee the minimum, and the function

$$
P=\int_{V} \sigma d V, \int_{V} \Psi d V, \int_{V} \Phi d V
$$


representing the global dissipations are simultaneous Lyapunov functions as well.

With this the Glansdorff-Prigogine's principle could be summarized in mathematical forms like

$$
\begin{aligned}
& \frac{d P}{d t}=\frac{d}{d t} \int_{V} \sigma d V \leq 0, \frac{d}{d t} \int_{V} \Psi d V \leq 0, \frac{d}{d t} \int_{V} \Phi d V \leq 0 \text { (evolutioncriterion), } \\
& \delta^{(1)} P=0, \delta^{(1)} \int_{V} \Psi d V=0, \delta^{(1)} \int_{V} \Phi d V=0 \quad \text { (stationerity criterion), } \\
& \delta^{(2)} P \geq 0, \delta^{(2)} \int_{V} \Psi d V \geq 0, \delta^{(2)} \int_{V} \Phi d V \geq 0 \quad \text { (stability criterion) }
\end{aligned}
$$

where $\delta^{(1)}, \delta^{(2)}$ are the first and second variations introduced in the first part of this article. In the following we will consider only the second variations, showing that the criteria in the third row of (202) are equivalent. For simplicity we use the short calculus, which we introduced above and show that the strictly convex character of potentials assures the positive definite second variation $\delta^{(2)} P=\left\langle\delta X_{i}, \delta j_{i}\right\rangle \geq 0$ of GEP

$$
\begin{aligned}
& j_{i}=\frac{\partial \Psi}{\partial X_{i}} \rightarrow \delta j_{i}=\delta \frac{\partial \Psi}{\partial X_{i}}=\frac{\partial^{2} \Psi}{\partial \Gamma_{j} \partial X_{i}} \delta \Gamma_{j}+\frac{\partial^{2} \Psi}{\partial X_{j} \partial X_{i}} \delta X_{j}=\frac{\partial^{2} \Psi}{\partial X_{j} \partial X_{i}} \delta X_{j} \rightarrow \\
& \rightarrow \delta^{(2)} P=\left\langle\delta j_{i}, \delta X_{i}\right\rangle=\left\langle\frac{\partial^{2} \Psi}{\partial X_{j} \partial X_{i}} \delta X_{j}, \delta X_{i}\right\rangle=\delta^{(2)} \int_{V} \Psi d V \geq 0 \\
& X_{i}=\frac{\partial \Phi}{\partial j_{i}} \rightarrow \delta X_{i}=\delta \frac{\partial \Phi}{\partial j_{i}}=\frac{\partial^{2} \Phi}{\partial \Gamma_{j} \partial j_{i}} \delta \Gamma_{j}+\frac{\partial^{2} \Phi}{\partial j_{j} \partial j_{i}} \delta j_{j}=\frac{\partial^{2} \Phi}{\partial j_{j} \partial j_{i}} \delta j_{j} \rightarrow \\
& \rightarrow \delta^{(2)} P=\left\langle\delta j_{i}, \delta X_{i}\right\rangle=\left\langle\frac{\partial^{2} \Phi}{\partial j_{j} \partial j_{i}} \delta j_{j}, \delta j_{i}\right\rangle=\delta^{(2)} \int_{V} \Phi d V \geq 0
\end{aligned}
$$

where we used the Donkin's theorem (36) and that the Hessian matrices of potentials are positive definite, (see for further proof the Appendix 7.3.).

Possibilities of the various stabilities of a steady-state system is shown on Fig. 3. A spontaneous fluctuation or perturbation can deviate the system from its stable steady-state in both directions. In consequence, the system has a new state by the fluctuation or perturbation, which would be the initial-state of the evolution after the disturbance. If, in this new state the entropy production is larger, then the evolution of the system will decrease the entropy production according to the first relation of (202) and the system returns to its original state. This case is shown in case of large perturbations by the $X_{i 1}$ state of the Figure 3., or in stationer case by $X_{i 2}$ state, when the perturbations are small enough. In case of the perturbed state, when the entropy production is smaller than it was in its original state, the evolution of the system will further decrease the entropy production, and continuously moves away from the initial stationer state governed by the first relation of (202). This happens in stationer state $X_{i 3}$.

The stability of a stationer state against any perturbations could be determined from dissipation potentials, because for a strictly convex potential, the availability of global 
minimum is guaranteed by availability of the local minimum; (for a proof see Appendix 7.4.). This condition guarantees that the state like $X_{i 2}$ cannot appear in constitutive theory. This circumstance has two important consequences. First is that the stationer state is stable, and second is that the system starting in any neighboring states of the final stationer one always reaches the same final state by its evolution. Shortly: these systems (in this general formulation also) show the Bertalanffy's equifinal behavior [30], [31].

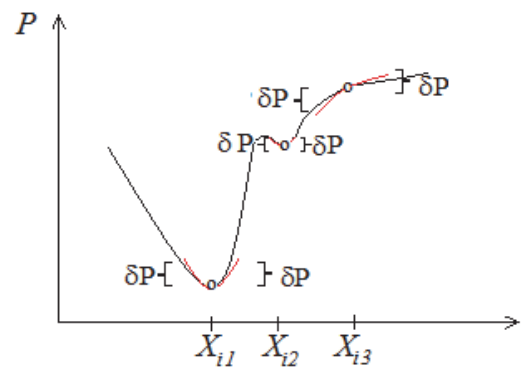

Fig. 3. Stable stationer state at $X_{i 1}$. A state at $X_{i 2}$ which is stable to small fluctuations but unstable to fluctuations which exceed some threshold amplitudes. Instable state at $X_{i 3}$.

\subsection{Examples}

\subsubsection{Homeostasis, equifinality and $1 /$ f scaling}

It is proven above, when the dissipation potentials are homogeneous Euler's functions in the frame of generalized Onsager constitutive theory, then the GEP is governed by the Glansdorff-Prigogine's general evolution criterion. This guarantees two conditions:

1. From one side the stationer state with minimal global entropy production is stable, and

2. From other side, the Bertalanffy's equifinality principle is valid, due to the dissipation potentials are strictly convex functions. The arbitrary large fluctuations (in the frame of reasonable limits) the system remains stable, returns to its stationer state.

Let us study the homeostasis of the living systems as its stationer state, accepting the concept of Nicolis and Prigogine, that the reference state of homeostasis should be regarded as a non-equilibrium steady- state [ ${ }^{32}$. The homeostasis as thermodynamic steady-state is controlled by hypothalamus, regulated by the sympathetic and the parasympathetic nervous system, as the action processor [33]. More precisely, the homeostatic state is caused by the intricate balance between antagonistic activity of the sympathetic and the parasympathetic nervous system [34], [33]. The actual value of the physical character is governed by the homeostasis $X(t)$ composed of a time-independent steady state $X_{S}$ and a time-dependent stochastic fluctuation $V(t)$. This last one is created commonly by the outside perturbations and the controller is independent from the initial conditions according to the Bertalanffy's equifinality principle.

In the following we discuss further two characters of the homeostasis, which could be obtained form the generalized results of [34]. They had shown, that a healthy heart rate obeys $1 / \mathrm{f}$ scaling. When this scaling property is valid for all the homeostasis; then

1. the necessary consequences of the homeostasis are the large fluctuations, and on the other hand, 
2. the $1 / \mathrm{f}$ scaling guarantees the constant (time independent) value of the deviation when the fluctuations could be characterized by their autocorrelation function in statistical point of view.

We shall show these points in the next parts. Supposing the homeostatic signal around the stationer state is

$$
V(t)=X(t)-X_{S}
$$

And its fluctuation is a self-similar stochastic signal, can be characterized by its autocorrelation function form statistical point of view. The self-similarity in this case means that the deviation of the fluctuations is

$$
\left\langle V^{2}(t)\right\rangle=t^{2 H}
$$

where $H$ is the Hurst' exponent of the self-similarity [35], [36]. For example when $H=1 / 2$, than

$$
\left\langle V^{2}(t)\right\rangle=t
$$

In this case the fluctuation is the noise of the random walk (Brownian) motion. The scaling law from (205) is

$$
\left\langle V^{2}(r t)\right\rangle=r^{2 H}\left\langle V^{2}(t)\right\rangle
$$

The noise could be characterized not only by the exponent of self-similarity, but by its spectral power density as well [37]:

$$
S(f)=F[G(\Delta t)]
$$

where

$$
G(\Delta t)=\langle V(t) V(t+\Delta t)\rangle-\left\langle V(t)^{2}\right\rangle
$$

is the autocorrelation function of the homeostasis noise and $F[G(\Delta t)]$ is the Fourier transform of this autocorrelation function. The spectral power density of the self-similar noises is [36], [37]:

$$
S(f) \propto \frac{1}{f^{\beta}}
$$

where $f$ is the frequency. Consequently, the power density of the self-similar signal is also self-similar, by exponent of $(-\beta)$. In case of $1 / f$ scaling law, from (210):

$$
\lim _{f \rightarrow 0} S(f) \propto \lim _{f \rightarrow 0} \frac{1}{f} \rightarrow \infty
$$


Hence the amplitude of the low frequency components of the noise could be extremely large, pushing far away the system from the $X_{S}$ state, which anyway is required to control the homeostasis. This is why the system must have the property of Bertalanffy's equifinality. The noise "colority" exponent $\beta$, and the self-similarity exponent $H$ are not independent [34], [35]:

$$
\beta=2 H+1
$$

Consequently, in the case of $1 / f$ scaling law

$$
H=0
$$

Hence, in consequence of (205), the deviation of this noise is time-independent. From this point of view, especially interesting the results of Struzik et al [34]: „modifying the relative importance of either of the two branches of the autonomic nervous system leads to a substantial decrease in $1 / \mathrm{f}$ scaling. In particular, the relative parasympathetic suppression, both by congestive heart failure and by the parasympathetic blocker atropine, results in a substantial increase in the Hurst exponent $H$ and a shift of the power density spectrum $S(f)$ from $1 / \mathrm{f}$ towards random walk scaling: $1 / f^{\beta}, \beta>1$." If $\beta>1$, than, based on (212) the Hurst's exponent will be positive, and so the deviation of the fluctuation by (205) will be monotonic increasing function of time. This is/can be predicted by Chebishev's theorem [ $\left.{ }^{38}\right]$, [39] increasing probability leaving the domain where the Bertalanffy's equifinality property is valid.

\subsubsection{Universality of entropy production of living systems}

It is a widely accepted opinion, that by evolution the living systems are developing towards such optimal state, where the energy used for sustenance of the life and operation of the energy-distribution system is the smallest. This last fact/point was also mentioned in connection with Murray's law before. However, the evolution of the living systems has a universal thermodynamic behavior (Andresen et al [40]). Regarding the living system as a set of cells, where the cells born and die, and which gets its energy from an energy-distributor network to fulfill its functions. The state of the system could be characterized by the extensive parameters of number of the cells $N$ and internal energy $U$, for which the balance equations are

$$
\frac{d N}{d t}=I_{N}+\Sigma_{N}, \quad \frac{d U}{d t}=I+\Sigma_{U}
$$

Disregarding the cell-motility, the number of cells has no flux, so it has only an augment (cell born) and attenuate (cell death) factors

$$
\frac{d N}{d t}=K-D
$$

where $K$ is the born number rate of cells, and $D$ is the death number rate of cells. This last one obtains the constitutive equation [41],

$$
N=\lambda N
$$


where $\lambda$ is the specific rate of cell-death, and its reciprocal value $\lambda^{-1}=T$ is the average lifetime of the cells. With these the balance equation of the cell-number is

$$
\frac{d N}{d t}=K-\lambda N
$$

The $I$ energy delivered in a unit-time by the blood-flow to the organ and distributed by the capillaries is the metabolic flux. This energy serves mainly a double task: a part for the sustaining the system and a part for the generation of new cells

$$
I=N B+E P=N B+E\left(\frac{d N}{d t}+\lambda N\right)
$$

where $B$ is the metabolic rate of a single cell, and $E$ is the metabolic energy for the production of a single cell. From this the energy-balance follows

$$
\begin{aligned}
& E \frac{d N}{d t}:=\frac{d U}{d t}=I-N(B+\lambda E)=I-U\left(\frac{B}{E}+\lambda\right)= \\
& =I+\Sigma_{U}, U:=E N, \Sigma_{U}:=U\left(\frac{B}{E}+\lambda\right)
\end{aligned}
$$

A constitutive equation is valid for the metabolic flux [41]:

$$
I=B_{0} N^{\alpha}=\frac{B_{0}}{E^{\alpha}} U^{\alpha}
$$

where $B_{0 *}$ is the metabolic flux of a single cell, $\mid 1 / 2<\alpha<1$ is a scaling exponent, which characterizes the fractal structure of the vascularization of the actual system. This last $\alpha=3 / 4$, supposed to create optimal distribution network according to West $\left[{ }^{42}\right],\left[{ }^{43}\right],\left[{ }^{44}\right]$. With this the energy-balance will be a non-linear differential equation for internal energy

$$
\frac{d U}{d t}=a U^{\alpha}-b U, a:=\frac{B_{0}}{E}, \quad b:=\left(\frac{B}{E}+\lambda\right)
$$

The first term on the right side of the equation is the energy flux, while the second term is the dissipation of the energy $\left(\Sigma_{U}\right)$ in the unit time. (The solution of the energy-balance is a sigmoid function [37] can be given in the form [41]:

$$
\begin{aligned}
& \left(\frac{U}{U_{\infty}}\right)^{1-\alpha}=1-\left(1-\left(\frac{U_{0}}{U_{\infty}}\right)^{1-\alpha}\right) e^{-a t / 4 U_{\infty}^{1-\alpha}}=1-e^{-a t / 4 U_{\infty}^{1-\alpha}+\ln \left(1-\left(\frac{U_{0}}{U_{\infty}}\right)^{1-\alpha}\right)}=1-e^{-\tau}, \\
& \tau(t):=a t / 4 U_{\infty}^{1-\alpha}-\ln \left(1-\left(\frac{U_{0}}{U_{\infty}}\right)^{1-\alpha}\right)
\end{aligned}
$$


where $U_{\infty}$ is the energy in stationer final state, $U_{0}$ is the energy in the time of birth. According to (222) the $\left(\frac{U}{U_{\infty}}\right)^{1-\alpha}$ quantity is a universal function of $\tau(t)$ time (so called biological self-time), in each biological systems. Let us study the dissipative part of the energy-balance in stationer state. The entropy production is stationer state from (221) is

$$
P=\frac{b U_{\infty}}{T}=\frac{a U_{\infty}^{\alpha}}{T}
$$

where $T$ the temperature of the system, is supposed to be constant. Consequently, the scaling law of the entropy-production is

$$
P=\frac{a U_{\infty}^{\alpha}}{T}=\frac{a E^{\alpha}}{T m^{\alpha}}\left(m N_{\infty}\right)^{\alpha}=k_{p} M^{\alpha}, \quad M:=m N_{\infty}
$$

where $m$ is the mass of a single cell, $N_{\infty}$ is the number of cells in stationer state, and $k_{p}$ is a constant.

This means that the entropy change of the system due to the entropy production during $d t$ time is

$$
d S=P d t=k_{p} M^{\alpha} d t
$$

Having the same entropy change in biological self-time scale the entropy production is: $P_{b}=P \frac{d t}{d \tau}$. We get that in the scale of the biological self-time the entropy production per unit mass of the system is constant [40]:

$$
\begin{aligned}
& \frac{1}{M} P_{b}=\frac{P}{M} \frac{d t}{d \tau}=\frac{P}{M} \frac{4 U_{\infty}^{1-\alpha}}{a}=\frac{4 P}{a M}\left(\frac{E}{m}\right)^{1-\alpha} M^{1-\alpha}=\frac{4 k_{p} M^{\alpha}}{a M}\left(\frac{E}{m}\right)^{1-\alpha} M^{1-\alpha}= \\
& =\frac{4}{a}\left(\frac{E}{m}\right)^{1-\alpha} k_{p}=k
\end{aligned}
$$

Supposing that, due to the evolution process, this specific entropy production is optimal, also minimum, then we can conclude that the state, in which the entropy production is minimal, is realized on the biological self-time scale. This optimal state is independent of the optimum of the vascularization, because the result does not depend on $\alpha$. Due to the equality of the metabolic flux and the rate of energy dissipation in stationer state, the metabolic entropy flux (belonging to the metabolic flux) per unit mass is constant on the biological self-time scale. Also, we can suppose the nourishment intake (food consumption per unit time) is proportional to the metabolic flux, so the nourishment rate per unit mass is also a universal constant in the biological self-time scale.

Furthermore, supposing the universal life-span in the biological self-time scale for every living individuals, the life-span $\left(\tau_{\text {life }}\right)$ is also a universal constant on this scale. Based on (222) the life-span on the physical time-scale is 


$$
t_{\text {life }}:=\tau_{\text {life }} \frac{4}{a} U_{\infty}^{1-\alpha}=\tau_{\text {life }} \frac{4}{a}\left(\frac{m}{E}\right)^{1-\alpha} M^{1-\alpha}=k_{\text {life }} M^{1-\alpha}
$$

Supposing that the nourishment rate (NR) is proportional with the metabolic flux, so $T R \propto M^{\alpha}$ then the energy demand by the nourishment on the physical life-span of the living organisms is

$$
\begin{aligned}
& W_{\text {tot }}=N R t_{\text {life }}:=\tau_{\text {life }} \frac{4}{a} U_{\infty}^{1-\alpha}=\tau_{\text {life }} \frac{4}{a}\left(\frac{m}{E}\right)^{1-\alpha} M^{1-\alpha}= \\
& =N R k_{\text {life }} M^{1-\alpha}=k_{\text {tot }} M^{\alpha} M^{1-\alpha}=k_{\text {tot }} M
\end{aligned}
$$

This means that each unit mass of all the living organisms consumes the same amount of energy in their life-span. The nourishment rate is proportional to the product of the mass flux of nutrients and its chemical potential, so proportional to the flux of negentropy [45]. Consequently, the small living organisms have large, the large organisms have small negentropy flux, because according to (228) their mass density has the same negentropy consumption in their actual life-span.

\subsubsection{Autocatalytic chemical reactions}

The chemical reaction kinetics is a class of irreversible processes, which remains outside of the applicability area of the Onsager constitutive theory. Hence, the reaction kinetics is an ideal discipline to test the applicability of the generalized Onsager constitutive theory. We shall/are going to study the autocatalytic reactions in a different way other than $\left[{ }^{46}\right],\left[{ }^{47}\right]$ did it before, emphasizing the following:

- We show that these types of reactions could be described by a dissipation potential which are homogeneous Euler's function of degree 3 of the concentration of chemical components.

- We show that this potential is a Lyapunov function of the differential equation of reaction kinetics.

- We give criteria of the stability of the stationer state by means of the dissipation potential (as Lyapunov function).

- We show that in the case of small perturbations this stability criteria is identical with the stability criteria deduced from the positive definite property of the second variation of the global entropy production.

Consider the following homogeneous autocatalytic reaction in a volume $V$

$$
A+X \underset{k_{-1}}{\stackrel{k_{1}}{\rightarrow}} 2 X
$$

where the chemical component $A$ has $C_{A}$ constant concentration which is necessary to proceed in/to a stationer state. The rate of the reaction $\frac{d \xi}{d t}$ as a thermodynamic flux is proportional with the rate of the $C$ concentration of the chemical component $X[48],[46]$.

$$
\frac{d C}{d t}=\frac{1}{V} \frac{d \xi}{d t}
$$


The parameter conjugated to the rate of reaction in the entropy production is the affinity $A$ has the form

$$
\begin{aligned}
& A=\mu_{A}+\mu_{X}-2 \mu_{X}=\text { const }-R T \ln \gamma C, \\
& \mu_{X}=\mu_{X 0}+R T \ln \gamma C,
\end{aligned}
$$

where $\gamma$ the affinity coefficient [48]. The reaction rate can be described by the reaction kinetic equation

$$
\frac{1}{V} \frac{d \xi}{d t}=\frac{d C}{d t}=k_{1} C_{A} C-k_{-1} C^{2}
$$

and it is homogeneous Euler's function of degree 2. From this, the concentration in stationer state is

$$
C_{\text {stac }}=\frac{k_{1} C_{A}}{k_{-1}}
$$

This steady-state is an equilibrium state in thermodynamic point of view, where the entropy production has its absolute minimum which is zero. Let the difference of the actual concentration from its stationer state of component $X$ be $c$. In this case, following a simple calculation, the reaction kinetic equation (232) can be written in the following form

$$
\frac{d c}{d t}=-k_{1} C_{A} c-k_{-1} c^{2}
$$

Denote the deviation of the actual affinity from its stationer value by $\alpha$, then

$$
\alpha=-R T \ln \gamma\left(1+\frac{c}{C_{\text {stac }}}\right) \cong-\gamma \frac{R T}{C_{\text {stac }}} c
$$

where we assumed that the deviation is small. In this approach the entropy production is

$$
P=\frac{1}{T} \frac{d \xi}{d t} A \cong \frac{V}{T} \frac{d c}{d t} \frac{\gamma R T}{C_{s t a c}}(-c)=\frac{\gamma R V}{C_{\text {stac }}} \frac{d c}{d t}(-c)
$$

which shows that the concentration rate and the concentration itself could be regarded as thermodynamic flux and thermodynamic force, respectively. The reaction kinetic equation (232), which makes a connection between these variables, can be considered as constitutive equation of the system. With this, on the analogy of the second equation of (21) the force dissipation potential is

$$
\begin{aligned}
& \Psi\left(C_{A}, c\right)=\int_{0}^{1}(-c) \frac{d c}{d t}\left(C_{A}, \tau c\right) d \tau=\int_{0}^{1} c k_{1} C_{A}(\tau c)+k_{-1} c(\tau c)^{2} d \tau= \\
& =\frac{1}{2} k_{1} C_{A} c^{2}+\frac{1}{3} k_{-1} c^{3}
\end{aligned}
$$

We show, that this dissipation potential is a Lyapunov function for definite range of concentrations. (Properties of Lyapunov function is discussed in [49], [47], and in Appendix 
7.8. ) The dissipation function (237) is positive in the $-\frac{3}{2} C_{\text {stac }}<c<C_{\lim }$ interval of the concentration, where $C_{\lim }$ is a positive limit-concentration till the model is applicable, and it has its zero value only at $c=0$ steady-state point. The second variation of (237) and its positivity interval are

$$
\begin{aligned}
& \delta^{(2)} \Psi=\frac{\partial^{2} \Psi\left(C_{A}, c\right)}{\partial c^{2}}(\delta c)^{2}=\left(k_{1} C_{A}+2 k_{-1} c\right)(\delta c)^{2} \geq 0, \\
& -\frac{1}{2} C_{\text {stac }}<c<C_{\lim }
\end{aligned}
$$

Taking into account the reaction kinetic equation (232) in the time-derivative of the potential, then we get

$$
\frac{d \Psi}{d t}=\frac{\partial \Psi\left(C_{A}, c\right)}{\partial c} \frac{d c}{d t}=-\left(\frac{d c}{d t}\right)^{2} \leq 0
$$

It is also seem from the reaction kinetic equation (232), that except the $c=0$ steady-state point the rate of concentration has no more zero values in the interval of $-\frac{1}{2} C_{\text {stac }}<c<C_{\lim }$. Consequently, the dissipation potential is a Lyapunov function of the reaction kinetic equation (232) in the $-\frac{1}{2} C_{s t a c}<c<C_{\lim }$ interval, and it guarantees that its solution is asymptotically stable, i.e.

$$
\lim _{t \rightarrow \infty} c(t)=0
$$

In case of small perturbations the dissipation potential can be replaced by its second variation at the steady-state point

$$
\Psi\left(C_{A}, \delta c\right)=\delta^{(2)} \Psi=\left.\frac{\partial^{2} \Psi\left(C_{A}, c\right)}{\partial c^{2}}\right|_{c=0}(\delta c)^{2}=k_{1} C_{A}(\delta c)^{2} \geq 0,
$$

and so the reaction kinetic equation will be

$$
\begin{aligned}
& \frac{d \delta c}{d t}=\frac{\partial \Psi\left(C_{A}, \delta c\right)}{\partial(-\delta c)}=-2 k_{1} C_{A}(\delta c) \rightarrow \delta c=\delta c_{0} e^{-\frac{t}{\tau}}, \\
& \tau:=\frac{1}{2 k_{1} C_{A}}=\frac{1}{2 k_{-1} C_{\text {stac }}}
\end{aligned}
$$

Consequently, the system is stable for small perturbations too. From this equations and the (230) relation follows that

$$
\delta \frac{d \xi}{d t}=V \frac{d \delta c}{d t}=-V 2 k_{1} C_{A} \delta c
$$


On the other hand, the variation of affinity from (235) is

$$
\delta \alpha=-\delta\left[R T \ln \gamma\left(1+\frac{c}{C_{\text {stac }}}\right)\right]=-\gamma \frac{R T}{C_{\text {stac }}} \delta c
$$

Hence the second variation of the global entropy production is positive definite for all small perturbations, i.e.

$$
\delta^{(2)} P=\frac{1}{T} \delta \alpha \delta \frac{d \xi}{d t}=V 2 \gamma R \frac{k_{1} C_{A}}{C_{\text {stac }}}(\delta c)^{2} \geq 0
$$

Consequently, the stability criteria (202) is satisfied in case of small perturbations. This can be used to decide about the stability of the system, but only in case of small perturbations.

\subsection{Exercises}

4.3.1. Let $\Delta X$ be the deviation of the homeostasis signal from its stationer value $X_{S}$, till the equifinality is valid. Calculate the $P$ probability by Chebishev theorem, when the homeostasis signal exceeds the value $P<\frac{\left\langle V^{2}(t)\right\rangle}{\Delta X^{2}}$, where the $\Delta X$ is the deviation from the homeostatic $X_{S}$ stationer state. Explain the result.

4.3.2. Accomplish the above estimation when the homeostasis signal has Gauss' distribution. Compare the results.

4.3.3. Calculate the energy-balance equation when the living organism performs extra mechanical work.

4.3.4. According to H. Lin [50] the maximal mechanical work of an animal (e.g. when they must get away from enemies) has the same scaling law as the metabolic rate [51] has. Show in this case, that the validity of universality law of specific entropy -production is valid at the biologic self-time scale.

\subsection{Boundary layer of homogeneous systems with two state-variables, as steady-state system}

We shall study the system in Fig. 4 . by means of the minimum principle of the global entropy production. Let the system be a so called current-tube, which is a tube where the boundary surface is constructed by those vector-lines of the thermodynamic fluxes, which fit on a closed line (for example circle, polygon).

Moreover, let us assume a common current-tube for all the fluxes in the system. (This is always the case in the isotropic system.) As shown above, the minimum principle of the global entropy production is valid when the fluxes fulfill the source-free balance equations

$$
\nabla \cdot \mathbf{j}_{i}=0,(i=1,2,3, \ldots, N)
$$

Integrating (246) on the $V$ volume of the current tube and applying the Gauss's divergence theorem

$$
\int_{V} \nabla \cdot \mathbf{j}_{i} d V=\oint_{\Omega} j_{i n} d A=0,(i=1,2,3, \ldots, N)
$$




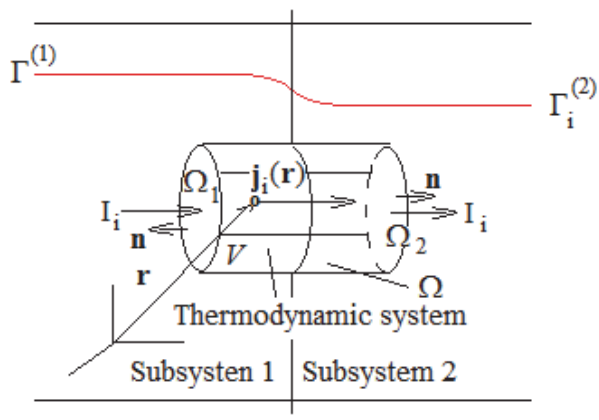

Fig. 4. Current-tube as thermodynamic system.

where $\Omega$ is the boundary of the system. Counting the vanished normal coordinates of fluxes on the boundary, the currents of the fluxes in the tube are:

$$
\begin{aligned}
& -\oint_{\Omega_{1}} j_{\text {in }} d A+\oint_{\Omega_{2}} j_{\text {in }} d A=0, \rightarrow-\oint_{\Omega_{1}} j_{\text {in }} d A=-\oint_{\Omega_{2}} j_{\text {in }} d A \\
& \rightarrow I_{i}:=-\oint_{\Omega_{1}} j_{\text {in }} d A=-\oint_{\Omega_{2}} j_{\text {in }} d A,(i=1,2,3, \ldots, N)
\end{aligned}
$$

The global entropy production of the current-tube is

$$
\begin{aligned}
& \int_{V} \nabla \Gamma_{i} \cdot \mathbf{j}_{i} d V=\int_{V} \nabla \cdot\left(\Gamma_{i} \mathbf{j}_{i}\right) d V=\oint_{\Omega} \Gamma_{i} j_{i n} d A= \\
& =-\oint_{\Omega_{1}} \Gamma_{i}^{(1)} j_{\text {in }} d A+\oint_{\Omega_{2}} \Gamma_{i}^{(2)} j_{\text {in }} d A=\left(\Gamma_{i}^{(1)}-\Gamma_{i}^{(2)}\right) I_{i}= \\
& =X_{i} I_{i}, X_{i}:=\left(\Gamma_{i}^{(1)}-\Gamma_{i}^{(2)}\right),(i=1,2,3, \ldots, N)
\end{aligned}
$$

where we assumed that the fluxes have no sources, and the intensive parameters are spacewise constants at the ends of the tube as we introduced the $X_{i}$ thermodynamic forces, which are the differences of the intensive parameters of the subsystems. The global entropy production is a bilinear form again, where the currents and forces are connected by the constitutive equations. Assume these constitutive equations belong to that type of generalized Onsager constitutive theory; where the dissipation potentials are homogeneous Euler's functions. In this case

$$
\begin{aligned}
& I_{i}=\frac{\partial \Phi\left(X_{j}\right)}{\partial X_{i}}, \quad X_{i}=\frac{\partial \Psi\left(I_{j}\right)}{\partial I_{i}},(i, j=1,2,3, \ldots, N) \\
& O\left(X_{j}, I_{j}\right):=\Phi+\Psi-X_{i} I_{i} \geq 0
\end{aligned}
$$

where the equality in the global OM-function is valid when the material equations of the first row are fulfilled. Moreover, for the relations among the global entropy production and the dissipation potentials, as we had seen before, we get 


$$
\begin{aligned}
& P=X_{i}\left(I_{j}\right) I_{i}=\frac{\partial \Phi\left(I_{j}\right)}{\partial I_{i}} I_{i}=k_{1} \Phi\left(I_{j}\right)= \\
& =X_{i} I_{i}\left(X_{j}\right)=X_{i} \frac{\partial \Psi\left(X_{j}\right)}{\partial X_{i}}=k_{2} \Psi\left(X_{j}\right),(i, j=1,2,3, \ldots, N) \\
& k_{1}^{-1}+k_{2}^{-1}=1
\end{aligned}
$$

where we used the second relation of (44).

Let us study two important cases:

- $\quad$ one is when we fix the first $k$ forces from all $N$ thermodynamic forces of the system. According to DeGroot referring on this stationer state of the system as the forceconstrained stationer state of degree $k\left[{ }^{52}\right]$,

- the second that we fix the first $k$ currents from all $N$ thermodynamic currents of the system. According to DeGroot referring on this stationer state of the system as the current-constrained stationer state of degree $k$ [52]

\subsection{Force-constrained stationer state}

Let the forces $X_{1}, \ldots, X_{k}$ be fixed and allow the forces $X_{k+1}, \ldots, X_{N}$ to vary. According to (251), in this case the force dissipation potential and the GEP depend on these variables and

$$
P\left(X_{j}\right)=k_{2} \Psi\left(X_{j}\right),(j=k+1,2,3, \ldots, N)
$$

Differentiate the GEP with respect to $X_{k+1}, \ldots, X_{N}$, then we obtain

$$
\frac{\partial P\left(X_{j}\right)}{\partial X_{j}}=k_{2} \frac{\partial \Psi\left(X_{j}\right)}{\partial X_{j}}=k_{2} I_{j}=0,(j=k+1,2,3, \ldots, N)
$$

which are the necessary conditions of the minimum of the global entropy production in a steady-state. Also fluxes conjugated to free thermodynamic forces vanish in steady-state, however the conjugated currents to the fix forces are not zero. These could be determined form the equations

$$
\left.\frac{\partial \Phi\left(I_{j}\right)}{\partial I_{j}}\right|_{I_{k+1}=\ldots I_{N=0}}=X_{j}=0,(j=1,2,3, \ldots, k)
$$

\subsection{Current constrained stationer state}

Let the fluxes $I_{k+1}, \ldots, I_{N}$ be prescribed and allow the forces $I_{k+1}, \ldots, I_{N}$ to vary. According to (251), in this case the current dissipation potential and the GEP depend on these variables and

$$
P\left(I_{j}\right)=k_{1} \Phi\left(I_{j}\right),(j=k+1,2,3, \ldots, N
$$

and 


$$
\frac{\partial P\left(I_{j}\right)}{\partial I_{j}}=k_{1} \frac{\partial \Phi\left(I_{j}\right)}{\partial I_{j}}=k_{1} X_{j}=0,(j=k+1,2,3, \ldots, N)
$$

Differentiate the GEP with respect to free fluxes $I_{k+1}, \ldots, I_{N}$, then we get the necessary conditions of the minimum of the GEP

$$
\left.\frac{\partial \Psi\left(I_{j}\right)}{\partial I_{j}}\right|_{I_{k+1}=\ldots=I_{N}=0}=X_{j}=0,(j=1,2,3, \ldots, k)
$$

Also, in this steady state the forces conjugate to free currents vanish. However, the conjugated currents to the fix forces are not zero. These could be determined form the equations

$$
\left.\frac{\partial \Psi\left(I_{j}\right)}{\partial I_{j}}\right|_{I_{k+1}=\ldots=I_{N}=0}=X_{j}=0,(j=1,2,3, \ldots, k)
$$

There are two extreme cases:

- when $k=0$ and so all the currents are zero in stationer case. This is the thermodynamic equilibrium, and the global entropy production has its absolute minimum.

- when $k=N$. This is a stationer state without freedom (no free parameters exist).

\subsection{Example: serial and parallel connections of current tubes}

\subsubsection{Serial circuit}

Consider the serial array shown in Fig. 5., where both the thermodynamic subsystems have identical thermodynamic currents, and its thermodynamic forces are additive values, because they are originated from the gradients of intensive parameters by line-integration). The dissipation potentials are given for each current-tube.

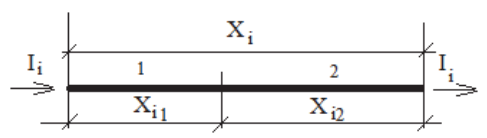

Fig. 5. Serial connection of the current-tubes.

The thermodynamic currents are identical in each current tube of the subsystems, so:

$$
\frac{\partial \Psi_{2}\left(X_{j 2}\right)}{\partial X_{i 2}}=-\frac{\partial \Psi_{2}\left(X_{j}-X_{j 1}\right)}{\partial X_{j 1}},(j=1,2,3, \ldots, N)
$$

Due to the fixed sums of the forces, the dissipation potentials depend on the thermodynamic forces of one of subsystems only; let us say the $1^{\text {st }}$ one. Using this

$$
\frac{\partial\left[\Psi_{1}\left(X_{j 1}\right)+\Psi_{2}\left(X_{j}-X_{j 1}\right)\right]}{\partial X_{i 1}}=0,(i, j=1,2,3, \ldots, N)
$$


Substituting these to (259) the result is

$$
X_{i 2}=X_{i}-X_{i 1}, \quad(i=1,2,3, \ldots, N)
$$

The forces belonging to the stationer state of the $1^{\text {st }}$ subsystem are the solutions of the above generally non-linear system of $N$ equations and with them the forces of the $2^{\text {nd }}$ subsystem are

$$
\begin{aligned}
& \Psi_{1}\left(X_{j 1}\right)+\Psi_{2}\left(X_{j 2}\right)=\min , \\
& X_{j 1}+X_{j 2}=X_{j}
\end{aligned}
$$

This result shows how the subsystems share the thermodynamic forces. What is the situation with the extremum property of GEP? The equations to solve this problem could be derived from the following constrained extremum-task

$$
P=I_{i} X_{i}=I_{i} X_{i 1}+I_{i} X_{i 2}=P_{1}+P_{2}
$$

and due to the additivity of the forces, the entropy production of the system is

$$
P_{l}=I_{i l} X_{i}=X_{i} \frac{\partial \Psi_{l}}{\partial X_{i}}=k_{\Psi l} \Psi{ }_{l}\left(X_{j}\right),(l=1,2),(i, j=1,2,3, \ldots, N)
$$

Using the equation expressing the connection between the entropy production and dissipation potentials

$$
P\left(X_{j 1}\right)=k_{\Psi 1} \Psi_{1}\left(X_{j 1}\right)+k_{\Psi 2} \Psi_{2}\left(X_{j}-X_{j 1}\right)
$$

We get from (265)

$$
\begin{aligned}
& \frac{\partial P\left(X_{j 1}\right)}{\partial X_{i 1}}=k_{\Psi 1} \Psi_{1}\left(X_{j 1}\right)+k_{\Psi 2} \Psi_{2}\left(X_{j}-X_{j 1}\right)= \\
& =k_{\Psi 1} \frac{\partial\left[\Psi_{1}\left(X_{j 1}\right)+\Psi_{2}\left(X_{j}-X_{j 1}\right)\right]}{\partial X_{i 1}}+\left(k_{\Psi 2}-k_{\Psi 1}\right) \frac{\partial \Psi_{2}\left(X_{j}-X_{j 1}\right)}{\partial X_{i 1}}= \\
& =\left(k_{\Psi 2}-k_{\Psi 1}\right) \frac{\partial \Psi_{2}\left(X_{j}-X_{j 1}\right)}{\partial X_{i 1}}, \quad(i, j=1,2,3, \ldots, N)
\end{aligned}
$$

where $k_{\Psi l}$ are the degrees of the homogeneous Euler's functions. From this we get

$$
\begin{aligned}
& \frac{\partial P\left(X_{j 1}\right)}{\partial X_{i 1}}=k_{\Psi 1} \Psi_{1}\left(X_{j 1}\right)+k_{\Psi 2} \Psi_{2}\left(X_{j}-X_{j 1}\right)= \\
& =k_{\Psi 1} \frac{\partial\left[\Psi_{1}\left(X_{j 1}\right)+\Psi_{2}\left(X_{j}-X_{j 1}\right)\right]}{\partial X_{i 1}}+\left(k_{\Psi 2}-k_{\Psi 1}\right) \frac{\partial \Psi_{2}\left(X_{j}-X_{j 1}\right)}{\partial X_{i 1}}= \\
& =\left(k_{\Psi 2}-k_{\Psi 1}\right) \frac{\partial \Psi_{2}\left(X_{j}-X_{j 1}\right)}{\partial X_{i 1}}, \quad(i, j=1,2,3, \ldots, N)
\end{aligned}
$$


where we used the (261) equations.

These calculations have two important consequences:

1. In the serial connection of the current tubes, the sum of the force potentials will be minimal in the stationer state of the system.

2. The GEP of the system in stationer case could be minimal then and only then when the dissipation potentials of the subsystems are homogeneous Euler's functions with identical degree.

\subsubsection{Parallel connection}

Consider a parallel array shown in Fig. 6., where both the thermodynamic subsystems have identical thermodynamic forces, and its thermodynamic currents are additive values. The dissipation potentials are given for each current-tube.

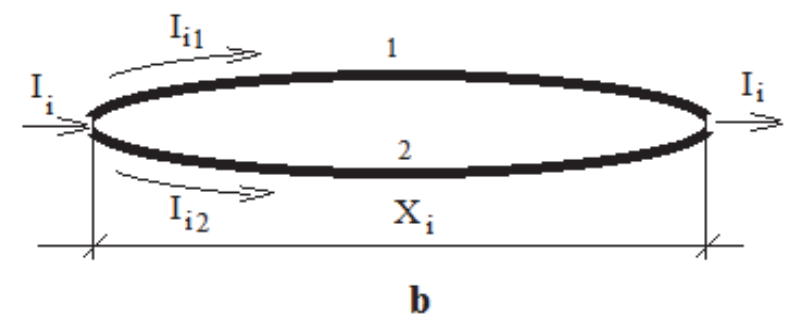

Fig. 6. Parallel connection of the current-tubes.

Because the thermodynamic forces are identical in the current tubes of the subsystems, so

$$
\frac{\partial \Phi_{2}\left(I_{j 2}\right)}{\partial I_{i 2}}=-\frac{\partial \Phi_{2}\left(I_{j}-I_{j 1}\right)}{\partial I_{i 1}},(i, j=1,2,3, \ldots, N)
$$

Due to the fixed sum of the currents, the dissipation potentials depend on the thermodynamic current of one of subsystems only; let us say this is the 1st one. Using this, we get

$$
\frac{\partial\left[\Phi_{1}\left(I_{j 1}\right)+\Phi_{2}\left(I_{j}-I_{j 1}\right)\right]}{\partial I_{j 1}}=0,(j=1,2,3, \ldots, N)
$$

Substituting these to (268) the result is

$$
I_{i 2}=I_{i}-I_{i 1}, \quad(i=1,2,3, \ldots, N)
$$

The currents belonging to the stationer state of the $1^{\text {st }}$ subsystem are the solutions of the above (270) generally non-linear system of $N$ equations and with them the currents of the 2nd subsystem are

$$
\begin{aligned}
& \Phi_{1}\left(I_{j 1}\right)+\Phi_{2}\left(I_{j 2}\right)=\min , \\
& I_{j 1}+I_{j 2}=I_{j}
\end{aligned}
$$


This result shows how the subsystems share the thermodynamic currents. What is the situation with the extremum property of GEP? The equations to solve this problem could be derived from the following constrained extremum-task

$$
P=I_{i} X_{i}=I_{i 1} X_{i}+I_{i 2} X_{i}=P_{1}+P_{2}
$$

The global entropy production of the system using the additivity of currents is

$$
\begin{aligned}
& P_{l}=I_{i l} X_{i}=X_{i} \frac{\partial \Psi_{l}}{\partial X_{i}}=k_{\Psi l} \Psi_{l}\left(X_{j}\right)= \\
& =\frac{k_{\Psi l}}{k_{\Psi l}-1} \Phi_{l}\left(I_{j l}\right)=k_{\Phi l} \Phi_{l}\left(I_{j l}\right),(l=1,2),(i, j=1,2,3, \ldots, N)
\end{aligned}
$$

Using the equations expressing the connection between the entropy production and dissipation potentials

$$
P=k_{\Phi 1} \Phi_{1}\left(I_{j 1}\right)+k_{\Phi 2} \Phi_{2}\left(I_{j 2}\right)
$$

and so the GEP of the system is

$$
P=k_{\Phi 1} \Phi_{1}\left(I_{j 1}\right)+k_{\Phi 2} \Phi{ }_{2}\left(I_{j 2}\right)
$$

Fix all the thermodynamic currents for the system. Due to the sums of the currents are prescribed, the entropy production depends on thermodynamic currents of one of subsystems only. Choose the $1^{\text {st }}$ one. Hence

$$
P\left(I_{j 1}\right)=k_{\Phi 1} \Phi{ }_{1}\left(I_{j 1}\right)+k_{\Phi 2} \Phi_{2}\left(I_{j}-I_{j 1}\right)
$$

From this we get the following result

$$
\begin{aligned}
& \frac{\partial P}{\partial I_{j 1}}=k_{\Phi 1} \frac{\partial \Phi_{1}\left(I_{j 1}\right)}{\partial I_{j 1}}+k_{\Phi 2} \frac{\Phi_{2}\left(I_{j}-I_{j 1}\right)}{\partial I_{j 1}}= \\
& =k_{\Phi 1} \frac{\partial\left[\Phi_{1}\left(I_{j 1}\right)+\Phi_{2}\left(I_{j}-I_{j 1}\right)\right]}{\partial I_{j 1}}+\left(k_{\Phi 2}-k_{\Phi 1}\right) \frac{\Phi_{2}\left(I_{j}-I_{j 1}\right)}{\partial I_{j 1}}= \\
& =\left(k_{\Phi 2}-k_{\Phi 1}\right) \frac{\Phi_{2}\left(I_{j}-I_{j 1}\right)}{\partial I_{j 1}},(j=1,2,3, \ldots, N)
\end{aligned}
$$

Where we used the equations (270).

These calculations have two important consequences:

1. In the parallel connection of the current tubes the sum of the current potentials will be minimal in the stationer state of the system.

2. The GEP of the system in stationer case could be minimal if and only if when the dissipation potentials of the subsystems are homogeneous Euler's functions with identical degree. 
The behavior of the sum of the dissipation potentials and the minimum property of GEP in generalized circuits are given in Appendix 7.5.

\subsection{Example}

Simple DC networks. The DC networks are typical steady-state systems where the minimum principle of global entropy production is valid. It is originated from two sources:

- $\quad$ The source-free stationer balances are valid for the fluxes (electric currents) expressed by the first Kirchhoff's law of.

- The thermodynamic forces could be originated from the gradients of the electric potential by line-integral, expressed by the Kirchhoff's second law.

However, further conditions are necessary also for the application of the constitutive theory.

- When the Ohm law is valid for the dissipative elements, then the dissipation potentials are Onsager's like.

- In case of non-linearity the dissipation potentials have to fulfill the requests of the generalized Onsager constitutive theory.

- Only two simple arrays will be discussed below: the serial and parallel DC-circuits in the frame of linear Onsager's theory.

\subsubsection{Potential distribution in serial circuit}

Our task is to determine the potential distribution in the serial circuit shown in Fig. 7., where the electric potentials are fixed in the $N_{1}, N_{2}$ points of the boundary so the sum of the thermodynamic forces is also fixed.

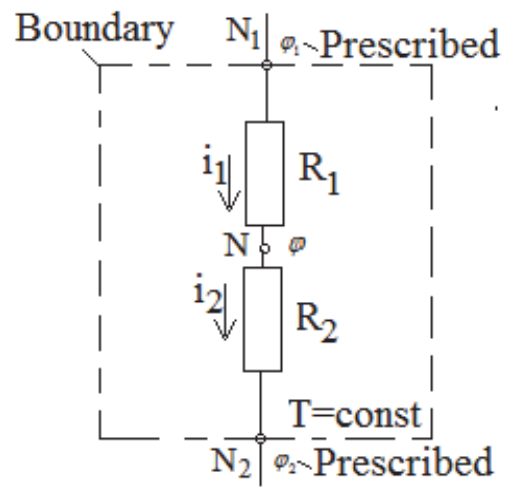

\section{Steady-state system}

Fig. 7. Simple serial DC-network, for what the minimum principle of global entropy production is valid.

Supposing that the system is isotherm, the dissipation potentials of the subsystems are

$$
\Psi_{1}(\varphi)=\frac{1}{2 T} \frac{\left(\varphi_{1}-\varphi\right)^{2}}{R_{1}}, \Psi_{2}(\varphi)=\frac{1}{2} \frac{\left(\varphi-\varphi_{2}\right)^{2}}{R_{2}}
$$


Knowing from the previous examples; the sum of the force potentials of the system will be the minimum in stationer state

$$
\Psi(\varphi)=\Psi_{1}(\varphi)+\Psi_{2}(\varphi)=\frac{1}{2 T} \frac{\left(\varphi_{1}-\varphi\right)^{2}}{R_{1}}+\frac{1}{2 T} \frac{\left(\varphi-\varphi_{2}\right)^{2}}{R_{2}}
$$

Hence the necessary condition for the minimum is

$$
\frac{d P(\varphi)}{d \varphi}=\frac{2}{T}\left(\frac{\left(\varphi-\varphi_{2}\right)}{R_{2}}-\frac{\left(\varphi_{1}-\varphi\right)}{R_{1}}\right)=0
$$

We can eliminate the potential $\varphi$ with the constraint on the electric potential differences like: $\left(\varphi_{1}-\varphi\right)+\left(\varphi-\varphi_{2}\right)=\left(\varphi_{1}-\varphi_{2}\right)$. Consequently, the potential distribution on the resistors will be

$$
u_{1}:=\varphi_{1}-\varphi=\left(\varphi_{1}-\varphi_{2}\right) \frac{R_{1}}{R_{1}+R_{2}}, u_{2}:=\varphi-\varphi_{2}=\left(\varphi_{1}-\varphi_{2}\right) \frac{R_{2}}{R_{1}+R_{2}}
$$

In this state the GEP of the system is minimal, because both the dissipation potentials are homogeneous Euler's functions of degree 2.

\subsubsection{Current distribution in parallel circuit}

Supposing again, that the system shown in Fig. 8. is isotherm, and on its boundary the current is fixed.

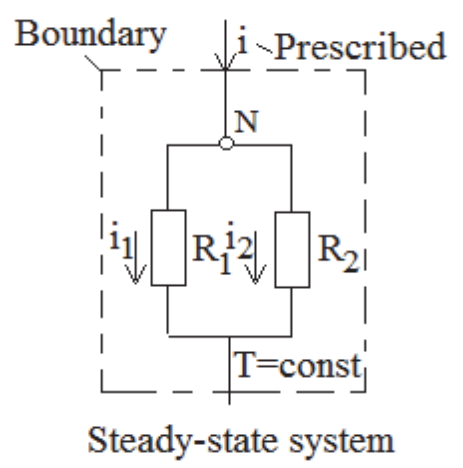

Fig. 8. Simple parallel DC-network, for what the minimum principle of global entropy production is valid.

The current potentials of the system are

$$
\Phi_{1}\left(i_{1}\right)=\frac{1}{2 T} i_{1}^{2} R_{1}, \Phi_{2}\left(i_{2}\right)=\frac{1}{2 T} i_{2}^{2} R_{2}
$$

And so from the previous example the sum of the current potentials of the system will be the minimum in stationer case 


$$
\Phi\left(i_{1}\right)=\Phi_{1}\left(i_{1}\right)+\Phi_{2}\left(i-i_{1}\right)=\frac{i_{1}^{2} R_{1}+\left(i-i_{1}\right)^{2} R_{2}}{2 T}
$$

From this the current distribution will be determined by the necessary condition of the minimum

$$
\frac{d \Phi\left(i_{1}\right)}{d i_{1}}=0, \rightarrow i_{1} R_{1}-\left(i-i_{1}\right) R_{2}=0, \rightarrow i_{1}=i \frac{R_{2}}{R_{1}+R_{2}}, i_{2}=i \frac{R_{1}}{R_{1}+R_{2}}
$$

Due to both dissipation potentials are homogeneous Euler's functions of degree 2, the GEP of the system is minimal in this state.

\subsection{Exercises}

4.9.1. Show in case of the current- tube, that $X_{i}:=\Gamma_{i}^{(1)}-\Gamma_{i}^{(2)}=r_{i k} I_{k}, r_{i k}=\int_{1}^{2} \frac{R_{i k}}{A(s)} d s$, when $\mathbf{X}_{i}=R_{i k} \mathbf{j}_{k}, \mathbf{X}_{i}=\nabla \Gamma_{i}$, and $A(s)$ is the surface of the cross-section of the tube at the $s$ arclength and $d s$ is the arc-element of the axis of the tube.

4.9.2. At which resistance value of $R_{2}$ will have the power its maximal value on it, when it is a consumptive element of the circle in Figure7.? Calculate the efficacy as well.

4.9.3. At which resistance value of $R_{2}$ will be the power maximal, when it is a consumptive element of the circle in Figure 8.? Calculate the efficacy as well.

4.9.4. Calculate the potential-drop on a given resistivity in an arbitrary DC circuit. Prove that this task could be solved by the minimization of the sum of dissipation potentials or by the principle of minimal entropy production, like we did in the serial connection of the Figure 5., when the circuit regarding the contacts of the resistor is replaced with the Thevenin's potential source equivalent circuit [53].

4.9.5. Calculate the current-flow on a given resistivity on an arbitrary DC circuit. Prove that this task could be solved by the minimization of the sum of dissipation fluxes or by the principle of minimal entropy production, like we did in the case of parallel connection of the Figure 6., when the circuit regarding the contacts of the resistor is replaced with the Norton's current source equivalent circuit [53].

4.9.6. Calculate the results in the above two exercises, when the given resistor is non-linear. Which part of the above results remains correct?

\section{Dissipative energy converters; characteristic functions}

This topic is discussed only from the point of view of extremum principles. The couplings among the thermodynamic fluxes in standard and generalized Onsager constitutive theories make it possible that the energy could have numerous carriers simultaneously in its degradation process in a dissipative structure; and by this the various processes become energetically coupled, and the structure could work like an energy converter. Such converters in the biology for example are the muscle processes, the photochemical reactions in photosynthesis, the chemical reactions for ATP synthesis. Based on the constitutive theory, in the following we describe the general characterization of the energy converters. Based on the constitutive theory we show that the efficacy of the energy conversion depends on the ratio of the fluxes or the forces in a system which has two carriers only. 
A dissipative system could be energy converter, when not all the terms of the entropy production are positive, their sum is positive definite only. The problem will be studied in a dissipative system having two thermodynamic forces and currents and in case when the dissipation potentials are homogeneous Euler's function of degree $k$. In this case the currents and forces are connected with the following constitutive equations (see Appendix 7.6.)

$$
\begin{aligned}
& I_{1}=L_{11} X_{1}+L_{12} X_{2} \\
& I_{2}=L_{21} X_{1}+L_{22} X_{2}, \quad L_{12}=L_{21}
\end{aligned}
$$

where the conduction coefficients are homogeneous Euler's functions of degree $k-2$ of the forces. The entropy production is always positive, except in equilibrium, when the currents and forces have equally zero values. In this case the GEP can be presented as

$$
\begin{aligned}
& P=X_{1} I_{1}+X_{2} I_{2}=L_{11} X_{1}^{2}+2 L_{12} X_{1} X_{2}+L_{22} X_{2}^{2}= \\
& =\left(\sqrt{L_{11}} X_{1}+\frac{L_{12}}{\sqrt{L_{11}}} X_{2}\right)^{2}+\left(L_{22}-\frac{L_{12}^{2}}{L_{11}}\right) X_{2}^{2} \geq 0
\end{aligned}
$$

and thus

$$
L_{11}>0, \quad L_{22}>0, \quad L_{12}^{2}<L_{11} L_{22}, \text { for all } X_{1}, X_{2}
$$

Hence, the straight coefficients are positive, but the cross-coefficient may be both, positive or negative. This is in agreement with the experimental observation that coefficients like thermal or electrical conductivity are always positive while, for example thermo-electric conductivity coefficient is not definite. Supposing the $L_{12}$ cross-coefficient negative and the driver process have the index 2 . When the process is characterized by the $I_{2}$ current and by $X_{2}$ force, and the temperature $T$ of the system is constant (isotherm process); then this process takes $P_{\text {in }}:=T X_{2} I_{2}>0$ power from an energy source, while transforms from this a power of $T L_{12} X_{1} X_{2}<0$ value to the driven process denoted by 1 . This process will dissipate $T L_{11} X_{1}^{2}$ power, and the remaining $P_{\text {out }}:=T X_{2} I_{2}<0$ power could be used as source for other processes, it could be a supply of outside systems. The efficacy of the energy converter is defined in the standard manner

$$
\eta:=\frac{P_{\text {out }}}{P_{\text {in }}}=\frac{-T X_{1} I_{1}}{T X_{2} I_{2}}
$$

Introducing the entropy production and the dissipation potential into (286), then we get

$$
\eta=\frac{-X_{1} I_{1}}{X_{2} I_{2}}=1-\frac{P}{X_{2} I_{2}}=1-\frac{k \Psi\left(X_{1}, X_{2}\right)}{X_{2} I_{2}}
$$

The efficacy is maximal at constant primer power when the GEP is minimal in stationer state. However, this cannot be fulfilled in general, as we will show it later. Let us define the force ratio and coupling parameter to approach the classical discussion of Kedem and Caplan [54] in linear constitutive cases 


$$
x=\sqrt{\frac{L_{11}}{L_{22}}} \frac{X_{1}}{X_{2}}, q:=\frac{L_{12}}{\sqrt{L_{11} L_{22}}}<1, x \in[-1,0]
$$

Due to the conduction coefficients are homogeneous Euler's function of degree $k-2$ of the forces, we may request the universal dependence on the conduction coefficients as well, which are

$$
L_{i k}=L_{i k}^{0} g\left(X_{1}, X_{2}\right)
$$

The force ratio in this case linear function of the force ratio, and the coupling parameter is constant like Kedem-Caplan's theory. With the new denotations the (285) currents are

$$
\begin{aligned}
& I_{1}=\sqrt{L_{11} L_{22}} X_{2}(x+q) \\
& I_{2}=L_{22} X_{2}(q x+1)
\end{aligned}
$$

And so the efficacy of the converter is

$$
\eta(x, q)=\frac{-X_{1} I_{1}}{X_{2} I_{2}}=\frac{x(x+q)}{q x+1}
$$

Consequently, the efficacy depends on the force ratio and the coupling parameter only. The entropy production or the dissipation function (because our system is isotherm) is

$$
\Theta(x, q):=T P=T\left(X_{1} I_{1}+X_{2} I_{2}\right)=T L_{22} X_{2}^{2}\left(x^{2}+2 q x+1\right)
$$

This is another function of force ratio and the coupling parameter, so we do not expect the extremes in the two functions at the same value of the force ratio. This is one of the reasons some experts discuss, that not all biological systems maximize their efficacy, instead they try to optimize other "cost-function" depending its complexity and its connection on the surroundings [55]. In the following we shall try to summarize these functions and their roles. Additionally to the above mentioned two characteristic functions, the isotherm Power output function $\left(P_{\text {out }}\right)$ and the Ecological function $\left(E_{c}\right)$ are used. Their definitions are [55].

$$
\begin{aligned}
& P_{\text {out }}(x, q):=-T X_{1} I_{1}=-T L_{22} X_{2}^{2}(x+q) x, \\
& E_{c}(x, q):=P_{\text {out }}-\Theta=-T L_{22} X_{2}^{2}\left(2 x^{2}+3 q x+1\right)
\end{aligned}
$$

These functions are universal functions of the force ratio and coupling parameter refer to reference power $T L_{22} X_{2}^{2}$. We are going to show in the following, that the reference power does not depend on the force ratio in the linear Onsager constitutive theory, while it depends in the generalized Onsager constitutive theory. Using the (291) and the representation principle of homogeneous Euler's functions (see Appendix 7.7), we get

$$
L_{22}=L_{22}^{0} g\left(X_{1}, X_{2}\right)=L_{22}^{0}\left(\sqrt{\frac{L_{22}^{0}}{L_{11}^{0}}}\right)^{k-2}\left(\sqrt{\frac{L_{11}^{0}}{L_{22}^{0}}}\right)^{k-2} g\left(X_{1}, X_{2}\right)=
$$




$$
\begin{aligned}
& =L_{22}^{0}\left(\sqrt{\frac{L_{22}^{0}}{L_{11}^{0}}}\right)^{k-2} g\left(\sqrt{\frac{L_{11}^{0}}{L_{22}^{0}}} X_{1}, \sqrt{\frac{L_{11}^{0}}{L_{22}^{0}}} X_{2}\right)= \\
& =L_{22}^{0}\left(\sqrt{\frac{L_{22}^{0}}{L_{11}^{0}}}\right)^{k-2} X_{2}^{k-2} g\left(\sqrt{\frac{L_{11}^{0}}{L_{22}^{0}}} \frac{X_{1}}{X_{2}}, \sqrt{\frac{L_{11}^{0}}{L_{22}^{0}}}\right)= \\
& =L_{22}^{0}\left(\sqrt{\frac{L_{22}^{0}}{L_{11}^{0}}}\right)^{k-2} X_{2}^{k-2} g\left(x, \sqrt{\frac{L_{11}^{0}}{L_{22}^{0}}}\right)
\end{aligned}
$$

where we used that $g\left(X_{1}, X_{2}\right)$ is a homogeneous Euler's function of degree $k-2$. So the reference power is

$$
T L_{22} X_{2}^{2}=T L_{22}^{0}\left(\sqrt{\frac{L_{22}^{0}}{L_{11}^{0}}}\right)^{k-2} X_{2}^{k} g\left(x, \sqrt{\frac{L_{11}^{0}}{L_{22}^{0}}}\right)
$$

In the Onsager's linear constitutive theory $k=2$, so $g\left(X_{1}, X_{2}\right)$ does not depend on $x$, also it is constant, which we choose in 1 . In this case we receive the classical equation of the energy converters in which the efficacy does not depend on the force ratio, so the characteristic functions could be scaled by them without distortion. In general the reference power depends on the force ratio, so it is not suitable for the distortion-free scaling till separating the term which depends on the force ratio. In the proposed dissuasion the characteristic functions are

$$
\begin{aligned}
& P_{\text {out }}(x, q, m)=-T L_{22}^{0}\left(\sqrt{\frac{L_{22}^{0}}{L_{11}^{0}}}\right)^{k-2} X_{2}^{k} g\left(x, \sqrt{\frac{L_{11}^{0}}{L_{22}^{0}}}\right)(x+q) x \\
& \Theta(x, q, m)=T L_{22}^{0}\left(\sqrt{\frac{L_{22}^{0}}{L_{11}^{0}}}\right)^{k-2} X_{2}^{k} g\left(x, \sqrt{\frac{L_{11}^{0}}{L_{22}^{0}}}\right)\left(x^{2}+2 q x+1\right) \\
& E_{c}(x, q, m)=P_{\text {out }}-\Theta=-T L_{22}^{0}\left(\sqrt{\frac{L_{22}^{0}}{L_{11}^{0}}}\right)^{k-2} X_{2}^{k} g\left(x, \sqrt{\frac{L_{11}^{0}}{L_{22}^{0}}}\right)\left(2 x^{2}+3 q x+1\right), \\
& m:=k-2
\end{aligned}
$$

where $m$ is the degree of the $g$-function. From physical point of view it is trivial, that $g$ is positive homogeneous function of force ratio, which anyway has a negative value. Due to the degree $m$ is not necessarily integer, it could be a fraction, so we may assume that the $g$ depends on the power-function of the absolute value of the force ratio $x$ by

$$
g\left(x, \sqrt{\frac{L_{11}^{0}}{L_{22}^{0}}}\right) \propto|x|^{m}
$$

With this the characteristic functions by the new scaling concept are 


$$
\begin{aligned}
& P_{\text {out }}^{*}(x, q, m)=-x(x+q)|x|^{m} \\
& \Theta^{*}(x, q, m)=\left(x^{2}+2 q x+1\right)|x|^{m} \\
& E_{c}^{*}(x, q, m)=-\left(2 x^{2}+3 q x+1\right)|x|^{m} \\
& m:=k-2
\end{aligned}
$$

In the case of the classical Onsager constitutive theory the maxima of the characteristic functions do not appear at the same value of the force ratio as it was shown by AriasHernandez et al. in details [55]. Also, in this case the very simple explanation of the evolutionary selection would be that a biologic system evolves to the direction of the maximal efficacy together with the minimal dissipation is not fulfilled. However, in a work about the mitochondrial ATP oxidative phosphorysation Stucky [56] shows the way, which is in harmony with the above simple principle of evolutionary selection. Stucky assumed that mitochondrial ATP oxidative phosphorysation is in steady-state corresponding to minimum of GEP which simultaneously corresponds to a maximum efficiency state. To satisfy these conditions, Stucky introduces a third term into the GEP, corresponding to the attached cellular load, to where he fits a maximal output power. We have to study how we can satisfy the principle of evolutionary selection in generalized Onsager constitutive theory without extending of the GEP. Simple computing experiments can show the advantage of the new scaling concept proposed in (298), which "gives possibility" for evolution movements optimizing all the characteristic functions with respect to the force ratio at maximal efficacy by the proper choice of the power $m$. This is shown by the Fig. 9 . in the case of two different coupling parameters.
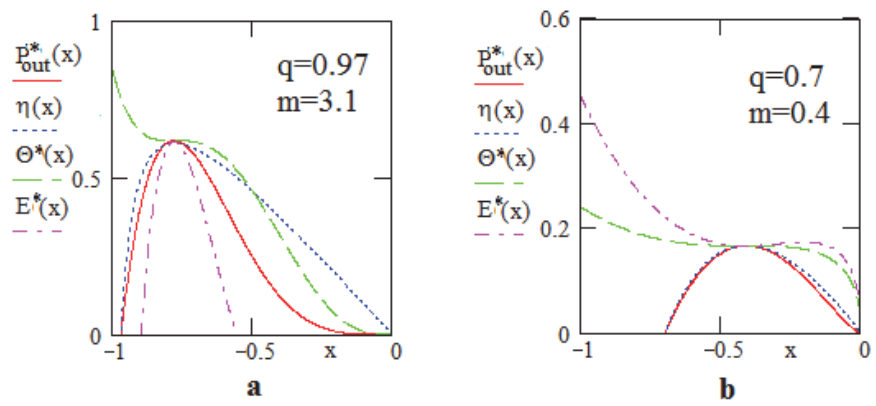

Fig. 9. The characteristic functions of the non-linear energy converters. $P$ is the output power, $\eta$ is the efficacy, $\Theta$ is the dissipation and $E$ is the ecologic function (the difference between the output power and the dissipation), $\mathrm{q}$ is the coupling parameter, and $\mathrm{m}$ is the power of the universal Euler's function in (297). These functions are not given on a common scale, they are on certain self-scales to show their behavior near the optimum state.

When $m=0$, the theory is linear, the classical theory is valid. This is the classical Onsager constitutive theory, which allows the quasi-linearity as well, when the coefficients could depend on the intensives, but not on their gradients. In this theory the minimum principle of global entropy production is not in harmony with the maximal efficacy, because the functions are independent. However, in non-linear constitutive theory the characteristic functions, as we see in Figure 9., are not in conflict with respect of simultaneous optimum. 
The Figure 9. shows, that in case of small coupling parameter a less non-linear system could be in a state of simultaneous optimum, and if the coupling parameter is large the simultaneous optimum comes only when the system is far away form the linearity. Finally, we note that this result is in agreement with the experimental studies of Nath [ $\left.{ }^{57}\right]$.

\section{On the principle of maximum entropy production}

There have been successful applications of the so called principle of maximum entropy production [58] for meteorological models, biological, environmental and ecological problems in the recent literature. H.Ziegler was the first who formulated this principle phenomenologically [59], substituting with this the Onsager's last dissipation of energy, which was proven in that time only for linear constitutive equations. The intention of Ziegler was to extend the non-equilibrium thermodynamical descriptions to the systems with non-linear constitutive equations. The validity of this maximum principle was proven by Zupanovi'c et.al for DC networks with well generalized conditions [ $\left.{ }^{60}\right]$. They applied this maximum entropy production principle in their model for bacterial photosynthesis for building the non-linear constitutive equation into their theory, [ ${ }^{61}$ ].Next, we shall discuss the maximum entropy production principle from the aspect of phenomenological thermodynamics only, and refer to its other applications, see for example [ ${ }^{62}$. The maximum entropy production principle does not contradict the principle of Onsager's last dissipation of energy in linear cases; on the contrary, they have equivalent value, [ $\left.{ }^{63}\right],\left[{ }^{64}\right]$. Some researchers state this equivalence as general (e.g. [ $\left.{ }^{65}\right]$ ), regarding two different, but identical formulations of the same natural principle. The equivalence is debated, and has not been proved till now. We would like to make steps forward the solution, without the final solution of the question; namely we show below the equivalence of the Ziegler's principle with the generalized Onsager's last dissipation of energy principle when the dissipation potentials are homogeneous Euler's functions. Like we did in the Appendix 7.2, let us start from the introduction of the force potential with the minimum task (38) of Legendre transform. Namely, using the definition (38), we get

$$
\Psi\left(X_{i}\right)=\max _{J_{i}}\left[X_{i} \cdot J_{i}-\Phi\left(J_{i}\right)\right]
$$

Based on this, the original Onsager's last dissipation of energy principle ensures the maximum of the function of the force potential, when the flux potential is homogeneous Euler's function of degree two. Due to the fact that (301) is valid in case of an arbitrary strictly convex flux dissipation potential, the generalized Onsager's last dissipation of energy principle could be formulated as

$$
\max _{J_{i}}\left[X_{i} \cdot J_{i}-\Phi\left(J_{i}\right)\right]
$$

This is a differential extremum principle. Let us prove the Ziegler's maximum entropy production principle, finding the possible mathematical form of the

$$
X_{i}=X_{i}\left(J_{1}, J_{2}, \ldots, J_{j}, \ldots, J_{n}\right),(i, j=1,2,3, \ldots, n)
$$

constitutive equations. If we substitute these equations into the entropy production, we get 


$$
\sigma\left(J_{i}\right)=J_{i} X_{i}=J_{i} X_{i}\left(J_{j}\right)
$$

which is a function defined on the linear vector space of fluxes $\mathbf{J}=\left(J_{1}, J_{2}, \ldots, J_{j}, \ldots, J_{n}\right)$. Ziegler proposed the following principle to find the possible form of the constitutive equations (303).

Ziegler's principle. If the forces $X_{i}$ are prescribed, the actual fluxes $J_{i}$, (which are satisfied the entropy production $\left.\sigma\left(J_{i}\right)=J_{i} X_{i}\right)$, maximize the function of entropy production (304), when the possible form of constitutive equations (303) are fulfilled as well. This principle can be formulated as a constrained maximum task, i.e.

$$
\begin{aligned}
& \sigma\left(J_{i}\right)=J_{i} X_{i}\left(J_{j}\right)=\text { extr }, \\
& \sigma\left(J_{i}\right)=J_{i} X_{i}
\end{aligned}
$$

By the Lagrange's theorem of multipliers can be constructed a free one

$$
\sigma\left(J_{i}\right)-\mu\left(\sigma\left(J_{i}\right)-J_{i} X_{i}\right)=\operatorname{extr}
$$

Necessary conditions belonging to this free extremum task are

$$
\begin{aligned}
& \frac{\partial\left[\sigma\left(J_{j}\right)-j\left(\sigma\left(J_{i}\right)-J_{i} X_{i}\right)\right]}{\partial J_{j}}=0,(i, j=1,2,3 \ldots, n) \\
& \frac{\partial\left[\sigma\left(J_{j}\right)-\mu\left(\sigma\left(J_{j}\right)-J_{i} X_{i}\right)\right]}{\partial \mu}=0
\end{aligned}
$$

From these equations we obtain the possible form of the constitutive equations and the multiplier

$$
\begin{aligned}
& X_{i}=\lambda \frac{\partial \sigma\left(J_{j}\right)}{\partial J_{i}}(i, j=1,2,3 \ldots, n) \\
& \lambda=\sigma\left(J_{j}\right)\left(J_{i} \frac{\partial \sigma\left(J_{j}\right)}{\partial J_{i}}\right)^{-1}, \lambda:=\frac{\mu-1}{\mu}
\end{aligned}
$$

Assume that the entropy production function two times continuously differentiable function with respect to fluxes. Then from the previous first equations and the Young-theorem of the analysis we get the generalized reciprocal relations

$$
\frac{\partial X_{i}}{\partial J_{k}}=\frac{\partial X_{k}}{\partial J_{i}}=\lambda \frac{\partial^{2} \sigma\left(J_{j}\right)}{\partial J_{k} \partial J_{i}}=\lambda \frac{\partial^{2} \sigma\left(J_{j}\right)}{\partial J_{i} \partial J_{k}},(i, j, k=1,2,3, \ldots, n)
$$

Also the entropy production as function of the fluxes shows a property like flux dissipation potential. Because of this we could choose the flux potential as

$$
\Phi\left(J_{j}\right):=\lambda \sigma\left(J_{j}\right)
$$


From the first equations of (308) the entropy production function is satisfied the following functional equation

$$
\sigma\left(J_{j}\right)=J_{i} X_{i}\left(J_{j}\right)=\lambda J_{i} \frac{\partial \sigma\left(J_{j}\right)}{\partial J_{i}}
$$

From this take into account Appendix 7.7., follows that the entropy production, as function of fluxes, is homogeneous Eulers's function of degree $\lambda$. Because of this property for the flux dissipation potential we obtain

$$
J_{i} \frac{\partial \Phi\left(J_{j}\right)}{\partial J_{i}}=J_{i} X_{i}\left(J_{j}\right)=\lambda^{-1} \Phi\left(J_{j}\right)
$$

Thus the flux potential is homogeneous Euler's function of degree $\lambda^{-1}$ as well. The flux potential in (301) is strict convex, thus $0<\lambda<1, \mu>0$ in (308). Substitute the flux potential to the Ziegler's principle (306) then after some algebra we obtain the generalized Onsager's last dissipation of energy principle (302), i.e.

$$
\begin{aligned}
& \sigma\left(J_{i}\right)-\mu\left(\sigma\left(J_{i}\right)-J_{i} X_{i}\right)=\max = \\
& =\frac{\Phi\left(J_{i}\right)}{\lambda}-\mu\left(\frac{\Phi\left(J_{i}\right)}{\lambda}-J_{i} X_{i}\right)=\mu\left[J_{i} X_{i}-\Phi\left(J_{i}\right)\right] \rightarrow J_{i} X_{i}-\Phi\left(J_{i}\right)=\max
\end{aligned}
$$

Also the Ziegler's principle is resulted in the same class of Onsager constitutive theory as was resulted by the minimum principle of GEP and in case of this class the generalized Onsager's last dissipation of energy principle and the (306) form of the Ziegler's principle is equivalent.

\section{Appendixes}

\subsection{The non-existence theorem of variational principle of a steady-state thermodynamic system}

The non-existence theorem of Gage-Schiffer-Kline-Reynolds [ ${ }^{66}$ ] had been neglected in the literature of irreversible thermodynamics like many other "non-convenient" complicated tasks, and on what Richardson focused again [67]. Formulate the problem with Richardson's original wordings:

They demonstrated [66] the impossibility of finding a universal extremal functional of the form (in one dimension),

$$
\Theta=\int_{A}^{B} \Phi\left(L_{i k}, \Gamma_{i}, \frac{d \Gamma_{i}}{d x}\right) d x
$$

where $\Phi$ is a specific function of its arguments, such as

$$
\delta \Theta=0
$$

is equivalent to the steady-state conservation equation 


$$
\frac{d j_{i}}{d x}=\frac{d}{d x} L_{i k} \frac{d \Gamma_{k}}{d x}=0
$$

Standard notation is used; $x$ denotes physical extent".

We note, the "non-existence theorem" is formulated for quasi-linear Onsager's constitutive theory, in which the $L_{i k}$ conduction coefficients could depend on the intensive parameters. Jaynes has the same conclusion in his discussion on the minimum principle of global entropy production [46]. However, the theory presented above allows such extremal functional which is free variation principle by the thermodynamic forces and, however restricted by the fluxes; and in which the Euler-Lagrange equations belonging to the variation of intensive parameters are equivalent with the stationer transport -equations. We may go further, construct from this partially restricted variational-task a free one by Lagrange's method of multipliers. The solution of the problem is available by the Donkin's theorem, for what firstly Gyarmati had focused on [ $\left.{ }^{68}\right]$, and he was the first who recognized $\left.{ }^{2}\right]$, that for the solution the variations must be done in the unified vector space of the intensives, forces and fluxes.

\subsection{Principle of last dissipation of energy}

The original Onsager's principle of last dissipation of energy is derivable by the presentation of the force potential with the minimum task (38) of Legendre's transform. Namely, using the definition (38) which was actualized on vector -processes, we get:

$$
\Psi\left(\nabla \Gamma_{i}, \Gamma_{l}\right)=\max _{\mathbf{J} \in G}\left[\nabla \Gamma_{i} \cdot \mathbf{j}_{i}-\Phi\left(\mathbf{j}_{i}, \Gamma_{l}\right)\right]
$$

The functional produced with the above differential principle of (317) is

$$
\begin{aligned}
& \int_{V} L\left(\nabla \Gamma_{i}, \mathbf{j}_{i}, \Gamma\right) d V, \\
& L\left(\nabla \Gamma_{i}, \mathbf{j}_{i}, \Gamma\right):=\nabla \Gamma_{i} \cdot \mathbf{j}_{i}-\Phi\left(\mathbf{j}_{i}, \Gamma_{l}\right)
\end{aligned}
$$

Due to the Onsager's principle of last dissipation of energy this functional is maximal with respect to variations of the fluxes $\mathbf{j}_{i}$. The necessary condition of the maximum are the constitutive equations, which are presented as Euler-Lagrange equations:

$$
\frac{\partial L}{\partial \mathbf{j}_{i}}=\nabla \Gamma_{i}-\frac{\partial \Phi\left(\mathbf{j}_{i}, \Gamma_{l}\right)}{\partial \mathbf{j}_{i}}=0
$$

The original Onsager's idea declared this as a variation principle of the constitutive theory.

\subsection{Second variation}

Calculating the second variation of the sum of the volume-integrated dissipation potentials, we get the following terms:

$$
\left\langle\left.\frac{\partial^{2} \Phi}{\partial \Gamma_{j} \partial \mathbf{j}_{i}}\right|_{\Gamma_{k 0}, \mathbf{j}_{j 0}} \delta \Gamma_{j}, \delta \mathbf{j}_{i}\right\rangle,\left\langle\left.\frac{\partial^{2} \Psi}{\partial \Gamma_{j} \partial \nabla \Gamma_{i}}\right|_{\Gamma_{k 0}, \mathbf{j}_{j 0}} \delta \Gamma_{j}, \delta \nabla \Gamma_{i}\right\rangle
$$


Because $\Phi$ and $\Psi$ are two times continuously derivable functions we obtain:

$$
\frac{\partial^{2} \Phi}{\partial \Gamma_{j} \partial \mathbf{j}_{i}}=\frac{\partial^{2} \Phi}{\partial \mathbf{j}_{i} \partial \Gamma_{j}}=\frac{\partial}{\partial \mathbf{j}_{i}} \frac{\partial \Phi}{\partial \Gamma_{j}}=\frac{\partial}{\partial \mathbf{j}_{i}}\left(-\frac{\partial \Psi}{\partial \Gamma_{j}}\right)=0
$$

where we had taken into account the Donkin's theorem (36). Also, we can see that the terms (320) do not give contributions to the second variation of GEP.

\subsection{Proof of the equivalence the local and global minimum of a strictly convex potential}

Proceed from the potential $\Psi(\mathbf{X})$, where $\mathbf{X}$ is a super-vector constructed by the thermodynamic forces as coordinates. When the $\mathbf{X}_{0}$ is a local optimum of $\Psi(\mathbf{X})$, we have $\Psi(\mathbf{Z}) \geq \Psi\left(\mathbf{X}_{0}\right)$ for any $\mathbf{Z}$ in some neighborhood $U(\mathbf{X})$ of $\mathbf{X}$. For any $\mathbf{Y} \quad \mathbf{Z}=\lambda \mathbf{X}+(1-\lambda) \mathbf{Y}$ belongs to $U(\mathbf{X})$ for $\lambda<1$ sufficiently close to 1 , and it follows from strictly convexity $\Psi(\mathbf{X})$ of that. From this after some algebra we get

$$
\lambda \Psi(\mathbf{X})+(1-\lambda) \Psi(\mathbf{Y}) \geq \Psi(\lambda \mathbf{X}+(1-\lambda) \mathbf{Y})=\Psi(\mathbf{Z}) \geq \Psi(\mathbf{X})
$$

\subsection{Current tube $\mathbf{n}$-port as steady-state thermodynamic system}

Until now we studied such current-tube networks, where every current or every force was common. This limit is abandoned, introducing the current-tube n-port, as thermodynamic system. This system is symbolically shown in Fig. 10.a., where the positive measuring directions of the forces and currents are also indicated. The request of source-free currents is satisfied by assuming loop-currents for each input two-poles. This condition means for example in the i-th two-pole, that the current flowing in the upper connection, flows out through the lower one. In the following we shall study a thermodynamic system, which is created by the connection of an $n$-port and an $n$ - $k$-port. Fix the first $k$ forces of the $n$-ports by the force sources as usual in the network-theory [ $\left.{ }^{69}\right]$ (see Fig. 10.b.).

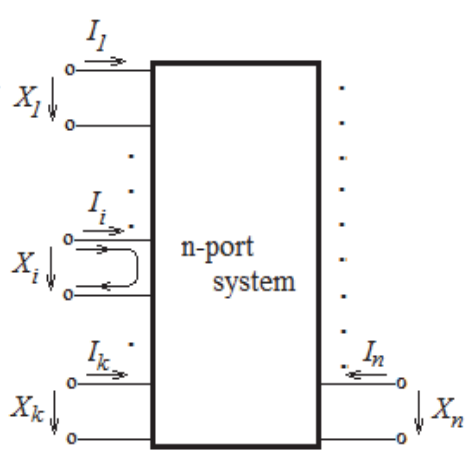

a

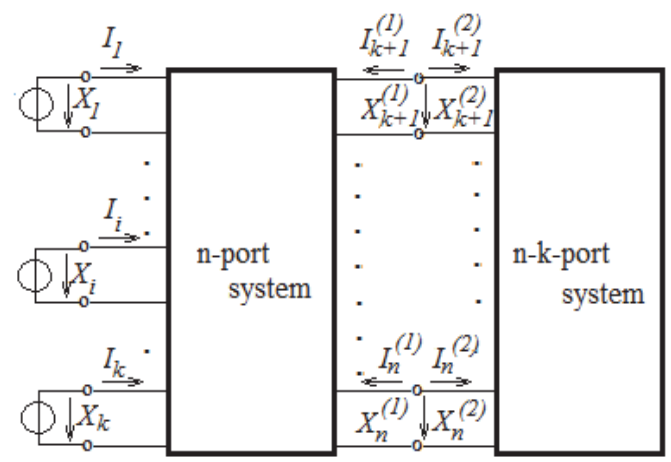

Force sources

b

Fig. 10. Current tube n-port as steady-state thermodynamic system. Connection of an n-port and a n-k-port. 
Give the force-potential for both the sub-systems. In this case the connection of the freeforces and currents can be formulated by the following non-linear equation

$$
\begin{aligned}
& I_{j}^{(1)}=\frac{\partial \Psi^{(1)}\left(X_{1}, ., X_{i}, ., X_{k}, X_{k+1}, \ldots, X_{l}, \ldots, X_{n}\right)}{\partial X_{j}}= \\
& =-I_{j}^{(2)}=\frac{\partial \Psi^{(2)}\left(X_{k+1}, \ldots, X_{l}, \ldots, X_{n}\right)}{\partial X_{j}},(j=k+1, \ldots, n)
\end{aligned}
$$

Rearranging its order, we get:

$$
\frac{\partial\left[\Psi^{(1)}\left(X_{1}, ., X_{i}, ., X_{k}, X_{k+1}, \ldots, X_{l}, \ldots, X_{n}\right)+\Psi^{(2)}\left(X_{k+1}, \ldots, X_{l}, \ldots, X_{n}\right)\right]}{\partial X_{j}}=0,
$$

Hence the free forces could be determined by the following constrained extremum- task:

$$
\begin{aligned}
& \Psi^{(1)}\left(X_{1}, ., X_{i}, ., X_{k}, X_{k+1}, \ldots, X_{l}, \ldots, X_{n}\right)+\Psi^{(2)}\left(X_{k+1}, \ldots, X_{l}, \ldots, X_{n}\right)=\min \\
& X_{i}=\text { prescribed },(i=1, \ldots, k)
\end{aligned}
$$

Using the following relation of the homogeneous Euler's function potentials and of the GEP of the sub-systems:

$$
P^{(l)}=k_{\Psi_{l}} \Psi^{(l)},(l=1,2)
$$

then we get for the total entropy production of the system

$$
P=k_{\Psi_{1}} \Psi^{(1)}+k_{\Psi_{2}} \Psi^{(2)}
$$

From this follows:

$$
\begin{aligned}
& \frac{\partial P}{\partial X_{j}}=k_{\Psi_{1}} \frac{\partial\left[\Psi^{(1)}+\Psi^{(2)}\right]}{\partial X_{j}}+\left(k_{\Psi_{2}}-k_{\Psi_{1}}\right) \frac{\partial \Psi^{(2)}}{\partial X_{j}}= \\
& =\left(k_{\Psi_{2}}-k_{\Psi_{1}}\right) \frac{\partial \Psi^{(2)}}{\partial X_{j}}
\end{aligned}
$$

Consequently, again we obtained the result that the sum of the dissipation potentials is minimal in stationer state and to the extremum of GEP is requested the identical degrees of Euler's function dissipation potentials. This means also, that using dissipation potentials has advantage in the thermodynamics of steady-state systems.

\subsection{Notes about the Hessian matrix}

A direct consequence of the possibility to represent the homogeneous Euler's functions by their partial derivative functions is that the thermodynamic currents and dissipation 
potentials can be described by the linear and quadratic form of the Hessian matrices. For example, if the force potential is a homogeneous Euler's function of degree $k$, then:

$$
\begin{aligned}
& \Psi\left(X_{1}, \ldots, X_{i}, \ldots, X_{n}\right)=\frac{1}{k} X_{i} \frac{\partial \Psi\left(X_{1}, \ldots, X_{i}, \ldots, X_{n}\right)}{\partial X_{i}}, \\
& \frac{\partial \Psi\left(X_{1}, \ldots, X_{i}, \ldots, X_{n}\right)}{\partial X_{i}}=\frac{1}{k-1} X_{j} \frac{\partial^{2} \Psi\left(X_{1}, \ldots, X_{i}, \ldots, X_{n}\right)}{\partial X_{j} \partial X_{i}}=I_{i}, \\
& \Psi\left(X_{1}, \ldots, X_{i}, \ldots, X_{n}\right)=\frac{1}{k(k-1)} \frac{\partial^{2} \Psi\left(X_{1}, \ldots, X_{i}, \ldots, X_{n}\right)}{\partial X_{j} \partial X_{i}} X_{i} X_{j}
\end{aligned}
$$

where we used that the partial derivative functions are one degree less homogeneous Euler's functions. When the dissipation potential has two variables only, then the currents:

$$
\begin{aligned}
& I_{1}=\frac{\partial \Psi\left(X_{1}, X_{2}\right)}{\partial X_{1}}=\frac{1}{k-1} X_{j} \frac{\partial^{2} \Psi\left(X_{1}, X_{2}\right)}{\partial X_{j} \partial X_{1}}=\frac{1}{k-1}\left(\frac{\partial^{2} \Psi}{\partial X_{1}^{2}} X_{1}+\frac{\partial^{2} \Psi}{\partial X_{2} \partial X_{1}} X_{2}\right)= \\
& =L_{11} X_{1}+L_{22} X_{2}, \\
& I_{2}=\frac{\partial \Psi\left(X_{1}, X_{2}\right)}{\partial X_{2}}=\frac{1}{k-1} X_{j} \frac{\partial^{2} \Psi\left(X_{1}, X_{2}\right)}{\partial X_{j} \partial X_{2}}=\frac{1}{k-1}\left(\frac{\partial^{2} \Psi}{\partial X_{1} \partial X_{2}} X_{1}+\frac{\partial^{2} \Psi}{\partial X_{2}^{2}} X_{2}\right)= \\
& =L_{21} X_{1}+L_{22} X_{2}, \\
& L_{11}:=\frac{1}{k-1} \frac{\partial^{2} \Psi}{\partial X_{1}^{2}}, L_{12}:=\frac{1}{k-1} \frac{\partial^{2} \Psi}{\partial X_{2} \partial X_{1}}=\frac{1}{k-1} \frac{\partial^{2} \Psi}{\partial X_{1} \partial X_{2}}=: L_{21}, L_{22}:=\frac{1}{k-1} \frac{\partial^{2} \Psi}{\partial X_{2}^{2}}
\end{aligned}
$$

where we introduced the conductivity coefficients, which are homogeneous Euler's functions of degree $\mathrm{k}-2$.

\subsection{Representation theorem of homogeneous Euler's function}

The representation theorem is a simple consequence of the definition of the homogeneous Euler's functions:

$$
\Psi\left(\lambda X_{1}, \ldots, \lambda X_{i}, \ldots, \lambda X_{n}\right)=\lambda^{k} \Psi\left(X_{1}, \ldots, X_{i}, \ldots, X_{n}\right)
$$

when $\lambda=X_{1}^{-1}, X_{1} \neq 0$, the result is the representation theorem (for a rigorous proof see [70]) theorem:

$$
\Psi\left(X_{1}, \ldots, X_{i}, \ldots, X_{n}\right)=X_{1}{ }^{k} \Psi\left(1, \ldots, \frac{X_{i}}{X_{1}}, \ldots, \frac{X_{n}}{X_{1}}\right)
$$

The proof that the partial derivative functions are one degree lower homogeneous Euler's functions could be shown as below. When the function is:

$$
I_{i}\left(X_{1}, \ldots, X_{i}, \ldots, X_{n}\right)=\frac{\partial \Psi\left(X_{1}, \ldots, X_{i}, \ldots, X_{n}\right)}{\partial X_{i}},(i=1,2,3, \ldots, N)
$$


then from this, by a few simple steps, we get the result

$$
\begin{aligned}
& I_{i}\left(\lambda X_{1}, \ldots, \lambda X_{i}, \ldots, \lambda X_{n}\right)=\frac{\partial \Psi\left(\lambda X_{1}, \ldots, \lambda X_{i}, \ldots, \lambda X_{n}\right)}{\partial \lambda X_{i}}=\lambda^{k} \frac{\partial \Psi\left(X_{1}, \ldots, X_{i}, \ldots, X_{n}\right)}{\partial \lambda X_{i}}= \\
& =\lambda^{k-1} \frac{\partial \Psi\left(X_{1}, \ldots, X_{i}, \ldots, X_{n}\right)}{\partial X_{i}}=\lambda^{k-1} I_{i}\left(\lambda X_{1}, \ldots, \lambda X_{i}, \ldots, \lambda X_{n}\right)
\end{aligned}
$$

\subsection{Lyapunov's theorem in the case of thermodynamic steady-state}

In this Appendix we study the Lyapunov's theorem in case of thermodynamic steady-state according to considerations of Schlogl [47] and Lassale\&Lefschetz [49].

When the changes of the steady-state thermodynamic system after the perturbations is the following system of differential equations:

$$
\frac{d X_{i}}{d t}=f\left(X_{1}, . ., X_{j}, . ., X_{N}\right), \quad(i, j=1,2,3, \ldots, N)
$$

and $\Psi\left(X_{j}\right)$ is a real function which exists in the whole state space $\mathbf{X}=\left(X_{1}, . ., X_{j}, . ., X_{N}\right)$ and is positive definite, i.e.

$$
\Psi(\mathbf{X}) \geq 0
$$

and the equality sign is valid at the steady state only. Moreover, let the second variation of $\Psi(\mathbf{X})$ be positive

$$
\delta^{(2)} \Psi(\mathbf{X})=\frac{\partial^{2} \Psi}{\partial X_{j} \partial X_{i}} \delta X_{i} \delta X_{j}
$$

in any state point, then we can state:

1. If exists a region in the state space which contains the steady-state point in its interior and if

$$
\frac{d \Psi(\mathbf{X})}{d t} \leq 0
$$

on the solution of differential equation (335), then the solution is stable whenever it starts in the interior of this region. The term stable means that the solution does not lead out of the limited neighborhood of the steady state point.

2. The solution is asymptotically stable if the equality sign in (338) is valid in the steady state point only. In this case the solution of the equation (335) leads finally into the steady state point.

In that case if (336) and (338) are valid in this region, then $\Psi(\mathbf{X})$ is called a Lyapunov function.

\section{Summary}

We showed above that rigorous conditions are necessary for the extremum principles of non-equilibrium steady-state. The limiting conditions could be categorized into two classes. 
The first one is the strong physical principles, expressed mathematically with balance equations. The other class is formed by the material or constitutive equations, describing the material behavior of the system. These equations however are always approximate. The possible general is only the reciprocal relation-principle, which was discovered on the linear constitutive equations by Onsager, and this is accepted in case of the non-linear processes as well. This was the way to the generalized Onsager constitutive theory. When this theory is applicable for the non-equilibrium steady-state system, then the Onsager's last dissipation of energy extremum principle could be generalized when the dissipation potentials derived from the reciprocal relations are strictly convex functions. We showed above that the minimum of entropy production could be derived from this generalized theory only when the balance equations are source-free. In this case however, no further conditions exist for dissipation potentials.

It is shown another way also to obtain the principle of minimal entropy production. Namely, we used the Glansdorff-Prigogine general evolution criterion, as Prigogine originally did. According to this principle the term of entropy production connected to the velocity of the thermodynamical forces monotonically decreases in time in non-equilibrium systems with time-independent boundary conditions, until the system reaches its equilibrium state, when that state exists. This is a strict principle because its validity does not depend on which constitutive theory governs the mentioned equations of the system. When the generalized Onsager constitutive theory in the point of view of the material equation of the given system belongs to such a sub-category where the dissipation potentials are homogeneous Euler's functions, then as it was shown above, Glansdorff-Prigogine general evolution criterion will be valid for all dissipation potentials and so for the entropy production too. Consequently, when steady-state exists, this belongs to the minimal entropy production. This is a generalization of the minimal entropy production principle, which was recognized for linear constitutive equations by Prigogine. It is clear that this principle is weaker than the original, but well useable for decision of the stability of the steady state. We showed above the existence of such steady-state systems where despite one of the global dissipative potentials is minimal, the global entropy production has no extremum properties.

At the end we proved the equivalence of the Ziegler's maximum entropy production principle with the generalized Onsager's last dissipation energy principle in cases when the dissipation potentials are homogeneous Euler's functions.

\section{Chart of symbols}

\begin{tabular}{|l|l|}
\hline Symbolum & Referencia \\
\hline$\nabla \Gamma_{\mathrm{i}}$ & i-th thermodynamic force (gradient of the intensive scalar $\Gamma_{\mathrm{i}}$ ) \\
\hline$A_{f}$ & affinity \\
\hline$a_{i}$ & extensive state variable \\
\hline $\mathbf{B}$ & magnetic flux density \\
\hline$B$ & metabolic rate of a single cell \\
\hline$C_{\text {stac }}$ & concentration in stationer state \\
\hline$D$ & death number rate of cells \\
\hline$D(O)$ & definition domain \\
\hline
\end{tabular}




\begin{tabular}{|c|c|}
\hline$D_{i k}(\alpha)$ & $\begin{array}{l}\text { tensors elements connect the chemical potential gradients and the } \\
\text { heat-flux }\end{array}$ \\
\hline$D_{i k}(\alpha \beta)$ & elements of diffusion tensor \\
\hline $\mathbf{E}$ & electric field-strength \\
\hline$E$ & metabolic energy for the production \\
\hline$E_{c}$ & ecological function \\
\hline$G(\Delta t)$ & autocorrelation function \\
\hline $\mathbf{H} \Phi\left(\mathbf{J}, \Gamma_{l}\right)$ & Hessian matrix \\
\hline $\mathbf{j}$ & electric current density \\
\hline $\mathbf{j}_{i}$ & current density vector of the i-th extensive \\
\hline $\mathbf{j}_{q}$ & heat-current density vector \\
\hline $\mathbf{j}_{s}$ & entropy current denstiy \\
\hline $\mathbf{j}_{\alpha}$ & mass-current density of the a-th component \\
\hline$k$ & specific energy dissipation per unit time \\
\hline$K$ & born number rate of cells \\
\hline $\mathrm{K}_{i k}(\mathrm{\alpha})$ & elements of heat conductivity tensor of $\alpha$-th of chemical components \\
\hline$L$ & Lagrangian \\
\hline $\mathbf{L}=\left[L_{i k}\right]$ & conductivity tensor \\
\hline$L_{i k}$ & conduction coefficients \\
\hline$m$ & mass \\
\hline $\mathrm{m}_{i}$ & mass of i-th component \\
\hline$N$ & number of the cells \\
\hline NR & nourishment rate \\
\hline$O\left(\nabla \Gamma_{j}, \mathbf{j}_{j}, \Gamma_{k}\right)$ & global OM-function \\
\hline$o\left(\mathbf{X}, \mathbf{J}, \Gamma_{l}\right)$ & Onsager-Machlup function \\
\hline$P$ & global entropy production \\
\hline$P_{\mathrm{deg}}$ & power of the irreversible processes \\
\hline$P_{\text {out }}$ & Power output function of disipative energy converter \\
\hline $\mathbf{R}$ & specific resistance tensor \\
\hline$r_{0}$ & mother-vessel with radius \\
\hline$r_{1}$ & daughter wessels \\
\hline$r_{\mathrm{i}}$ & radius \\
\hline$R_{i j}(\mathbf{B})$ & resistivity tensor \\
\hline$R_{i k}$ & specific heat-resistivity tensor \\
\hline$R_{\mathrm{jl}}\left(\Gamma_{k}\right)$ & kinetic coefficients \\
\hline$s$ & entropy density \\
\hline$S(f)$ & spectral power density of the homesotatic noise \\
\hline$T$ & absolute temperature \\
\hline$U$ & internal energy \\
\hline$U_{0}$ & energy in the time of birth \\
\hline$u_{1}$ & potential distribution \\
\hline$U_{\infty}$ & energy in stationer final state \\
\hline$V$ & volume \\
\hline$V(t)$ & homeostatic signal \\
\hline
\end{tabular}




\begin{tabular}{|l|l|}
\hline$W_{i o t}$ & energy demand \\
\hline $\mathbf{X}$ & 3 N dimensional thermodynamic force \\
\hline$X_{\mathbf{j}}=X_{\mathrm{j}}\left(j_{l} \Gamma_{k}\right)$ & $\mathrm{j}$-th cordinates of thermodynamic forces \\
\hline$a$ & ratio of radius \\
\hline$\beta$ & "colority" exponent of homeostatic noise \\
\hline$\gamma$ & affinity coefficient \\
\hline$\Gamma_{i}$ & i-th intensive scalar variable \\
\hline$\delta_{\mathrm{i}}$ & production term of the i-th extensive \\
\hline$\delta_{i k}$ & Kronecker-delta \\
\hline$\varepsilon_{i j k}$ & permutation symbol \\
\hline$\eta$ & efficacy \\
\hline$\Theta$ & dissipation function of dissipative energy converter \\
\hline$\lambda$ & special rate of cell-death \\
\hline$\Lambda(T)$ & specific heat conduction tensor \\
\hline$\lambda-1=T$ & average lifetime \\
\hline$\sigma$ & spontaneous internal entropy production \\
\hline$\tau_{l i f e}$ & life-span \\
\hline$\varphi$ & electric potential \\
\hline$\Phi\left(j_{l}, \Gamma_{k}\right)$ & flux dissipation potential \\
\hline$\Phi_{1}\left(i_{1}\right)$ & current dissipation potentials \\
\hline$\Psi\left(\mathbf{X}, \Gamma_{l}\right)$ & force dissipation potential \\
\hline$\Omega$ & boundary of the system \\
\hline
\end{tabular}

\section{References}

[1] Meixner J, Reik H (1959) Thermodynamik der irreversiblen Prozesse. Handbuch der Physik, III/2. Springer-Verlag Berlin-Göttingen-Heidelberg

[2] Gyarmati I (1970) Non-equilibrium Thermodynamics. Springer-Verlag Berlin-GöttingenHeidelberg

[3] Onsager L (1931) Reciprocal Relation in irreversible processes I. Phys. Rev. 37:405-426, Reciprocal Relation in irreversible processes II. Phys. Rev. 37: 2265-2279

[4] Edelen DGB (1937) On the existence of symmetry relations and dissipations potentials. Arch. Ration. Mech. Analysis 51218

[5] Casimir HBG (1945) On Onsager's principle of microscopic reversibility. Rev. Mod. Phys. 17:343-350

[6] Truesdell CA (1960) $\mathrm{Zu}$ den Grundlagen der Mechanik und Thermodynamik. Physikalische Bletter 16:512-517

[7] Berdichevsky VL (2003) Structure of equations of macrophysics. Physical Rewiew E 68, 066126

[8] Kolmogorov AN (1936) Zur Umkehrbarkeit der statistiechen Naturgesetze, Math. Ann. $113,766-772$

[9] Yaglom AM (1962) An introduction to the theory of stationary random functions, Prentice-Hall, Englewood Cliffs, N. J.,

[10] Spohn H (1991) Large Scale Dynamics of Interacting Particles. Springer-Verlag, Berlin 
[11] Berdichevsky V. (1997) Thermodynamics of Chaos and Order, Addison-WesleyLongman,

[12] Binmore \& Davies, (2007), Calculus Concepts and Methods, Cambridge University Press, p.190

[13] Rockefellar RT (1996) Convex Analysis. Princeton University Press

[14] Gantmacher F (1970) Lectures in Analytical Mechanics. Mir Publichers Moscow

[15] Arnold VI (1978) Mathematical Methods of Classical Mechanics. Springer-Verlag, New York

[16] Onsager L, Machlup S (1953) Fluctuations and irreversible Processes. Physical Rewiew 91(6):1505-1512

[17] Abramowitz, M. Stegun, IA. (1964), Handbook of Mathematical Functions, New York: Dover Publications

[18] Michlin SG (1962) Variationsmethoden der Mathematischen Physik. Akademie-VerlagBerlin

[19] Becker R (1951) Theorie Der Elektrizitat, Band 1. Teubner Verlagsgesellschaft-Leipzig, (1982) Electromagnetic field and Intaractions. Dover Publications, New-York

[20] Murray CD (1926) The Physiological Principle of Minimum Work. I. The Vascular System and Cost of Blood Volume. PNAS 12 pp 207-214

[21] Murray CD (1926) The Physiological Principle of Minimum Work Applied to the Angle of Branching of Arteries. J. Gen. Physiol. 9:835-841

[22] Yunlong Huo, Kassab GS (2009) A Scaling Law of Vascular Volume. Biophysical Journal 96:347-353

[23] West GB, Brown JH, Enquist BJ (1997) A general model for the origin of allometric scaling laws in biology. Science. 276:122-126

[24] Finlyason BA, Scriven LC (1967) On the search for variational principles. Int. J. Heat Mass Transfer 10:799-821

[25] Lebon G, Jou D, Casas-Vazquez J (2008) Understanding Non-equilibrium Thermodynamics. Springer-Verlag-Berlin-Heidelberg

[26] Gyarmati I (1971) Application of the Gyarmati principle to multicomponent nonisothermal and chemically reacting diffusional systems. Acta chim. Hung. 67: 303-320

[27] Jeans JH (1923) The Mathematical Theory of Electricity and Magnetism. Cambridge University Press, Cambridge, England

[28] Glansdorff P, Prigogine I (1954) Sur les Sur les propriétés différentielles de la production d'entropie. Physica 20:773-773

[29] Glansdorff P, Prigogine I (1971) Thermodynamic Theory of Structure, Stability and Fluctuations. John Wiley \& Sons, London, New York

[30] Bertalanffy L, (1968) General System theory: Foundations, Development, Applications, George Braziller, New York

[31] Bertalanffy L, (1962), Modern Theories of Development, New York: Harper

[32] Nicolis G, Prigogine I (1989) Exploring Complexity. Freeman, New-York

[33] Recordati G, Bellini TG (2004) Definition of internal constancy and homeostasis in the contex of non-equilibrium thermodynamics. Exp. Physiol. 89:27-38

[34] Struzik ZR, et al (2005) Dual Antagonistic Autonomic Control Necessary for 1/f Scaling in Heart Rate. In Fractals in Biology and Medicine Volume IV. Editors:G.A. Losa et al. Birkhauser Basel-Boston-Berlin 
[35] Mandelbrot BB (1983) The Fractal Geometry of Nature. Freeman, San Francisco

[36] Badii B, Politi A (1997) Complexity, Hierarchical structures and scaling in physics. Cambrige University Press, UK

[37] Musha, T., Sawada, Y. (eds.) (1994). Physics of the living state. IOS Press, Amsterdam

[38] Rényi A, (1966) Wahrscheinlichkeitsrechnung. VEB Deutscer Verlag der Wissenschaften Berlin

[39] Rozanov YA, (1985) Probability Theory, A Concise Course. Dover Publications, Inc. New York

[40] Andresen B, Shiner JS, Uehlinger DE (2002) Allometric scaling and maximum efficiency in physiological eigen time. 99(9):5822-5824

[41] West GB, Brown J.H, Enquist B.J (2001) A general model for ontogenic groth. Nature $413,628-631$

[42] West GB, Woodruff WH, Brown JH (2002) Allometric scaling of metabolic rate from molecules and mitochondria to cells and mammals. 99(1):2473-2478

[43] Brown JH, West JB (2000) Scaling in Biology. Oxford University Press

[44] West GB, Brown JH, Enquist BJ (1999) The Fourt Dimension of Life: Fractal Geometry and Allometric Scaling of Organisms. Science 284(4):1677-1679

[45] Schroedinger E. (1944) What is Life? Cambridge University Press, Cambridge , New York

[46] Ebeling W (1976) Strukturbildung bei Irreversiblen Prozessen. Eine Enführung in die Theory dissipativer Strukturen. Teubner-Verlag-Leipzig

[47] Schlögl F (1971) On Stability of Steady State. Z. Physik 243:303-310

[48] Prigogine I, Defay R (1954) Chemical Thermodynamics. Longman, Green \& Co., London

[49] Lassalle J, Lefschetz S (1961) Stability by Lyaponov's direct method. Academic Press Inc. New-York

[50] Lin H (1982) Foundamentals of zoological scaling. Am. J. Phys. 50(1)

[51] McMahon T (1973) Size and Shape in Biology. Science 179:1209-1209

[52] von SR de Groot (1951) Thermodynamics of irreversible processes. North-Holland Publ. Co. Amsterdam

[53] Don H Johnson (2003) Equivalent circuit concept: the voltage-source equivalent. Proceedings of the IEEE 91(4):636-640

[54] Caplan SR, Essig A (1983) Bioenergetics and Linear Non-equilibrium Thermodynamics. 1st edn. Harvard University Press, Cambridge

[55] Arias LA, Hernandez et al (2007) A first-order irreversible thermodynamic approach to a simple energy converter. arXIV: 0709.0321v1 [cond - math.stat - mech]

[56] Stucki JW (1989) The optimal efficiency and the economic degrees of coupling of oxidative phosphorisation. Eur. J. Biochem. 109:269-283

[57] Nath S (1998) A thermodynamic principle for the coupled bioenergetic processes of ATP synthesis. Pure \& Appl. Chem. 70(3):639-644

[58] Kleidon A, Lorenz RD (2004) Entropy Production by Earth System Processes. Nonequilibrium thermodynamics and the production of entropy: life, Earth, and beyond. Springer Verlag, Heidelberg

[59] Ziegler H (1963) An attemp to generalize Onsager's principle, and its significance for rheological problems. ZAMP, 9(5-6):748-763 
[60] Županovi'CP, Jureti'cD, Botrati'cS (2004) Kirchhoff's loop law and the maximum entropy production principle. Physical Review E 70:056108

[61] Juretíc D, Županovi'c P (2003), Photosynthetic models with maximum entropy production in irreversible charge transfer steps. Computational Biology and Chemistry 27:541-553

[62] Dewar RC (2005) Maximum entropy production and the fluctuation theorem. J. Phys. A: Math. Gen. 38

[63] Martyushev LM, Seleznev VD (2006), Maximum entropy production principle in physics, chemistry and biology. Physics Reports 426:1-45

[64] Zupanovic'P et al (2010), The Maximum Entropy Production Principle and Linear. Irreversible Processes. Entropy 12:996-1005

[65] Kleidon A, Malhi Y, Cox PM (2010) Introduction Maximum entropy production in environmental and ecological systems. Phil. Trans. R. Soc. B 365:1297-1302

[66] Gage DH, Schiffer M, Kline SJ, Reynolds WC (1966) In Non-Equilibrium Thermodynamics: Variational Techniques and Stability. R. J. Donnelly (ed) University of Chicago

[67] Richardson IW (1969) On the Principle of Minimum Entropy Production. Biophysical Journal Vol.9

[68] Gyarmati I (1869) On the "Governing Principle of Dissipative Processes" and its extension to Non-linear Problemss. Ann. D. Phys, 353-378

[69] Reza FM, Seely S (1959) Modern Network Analysis. McGraw-Hill Book Company. Inc. New York-Toronto-London

[70] Acel J (1961) Vorlesungen über Funktionalgleichungen und Ihre Anwendungen. VEB Deutscher Verlag der Wissenschaften Berlin 


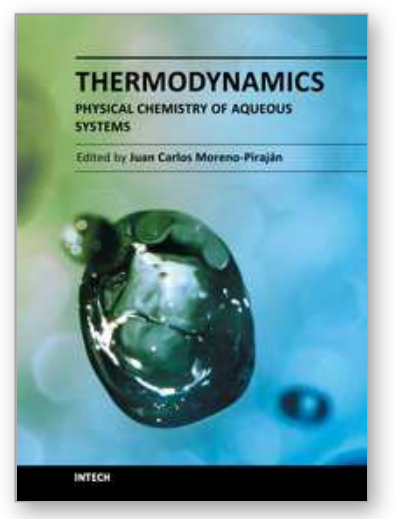

\author{
Thermodynamics - Physical Chemistry of Aqueous Systems \\ Edited by Dr. Juan Carlos Moreno Pirajễin
}

ISBN 978-953-307-979-0

Hard cover, 434 pages

Publisher InTech

Published online 15, September, 2011

Published in print edition September, 2011

Thermodynamics is one of the most exciting branches of physical chemistry which has greatly contributed to the modern science. Being concentrated on a wide range of applications of thermodynamics, this book gathers a series of contributions by the finest scientists in the world, gathered in an orderly manner. It can be used in post-graduate courses for students and as a reference book, as it is written in a language pleasing to the reader. It can also serve as a reference material for researchers to whom the thermodynamics is one of the area of interest.

\title{
How to reference
}

In order to correctly reference this scholarly work, feel free to copy and paste the following:

Gy. Vincze and A. Szasz (2011). On the Extremum Properties of Thermodynamic Steady State in Non-Linear Systems, Thermodynamics - Physical Chemistry of Aqueous Systems, Dr. Juan Carlos Moreno PirajÃ $i n ~(E d)$, ISBN: 978-953-307-979-0, InTech, Available from: http://www.intechopen.com/books/thermodynamicsphysical-chemistry-of-aqueous-systems/on-the-extremum-properties-of-thermodynamic-steady-state-in-nonlinear-systems

\section{INTECH}

open science | open minds

\section{InTech Europe}

University Campus STeP Ri

Slavka Krautzeka 83/A

51000 Rijeka, Croatia

Phone: +385 (51) 770447

Fax: +385 (51) 686166

www.intechopen.com

\section{InTech China}

Unit 405, Office Block, Hotel Equatorial Shanghai

No.65, Yan An Road (West), Shanghai, 200040, China

中国上海市延安西路65号上海国际贵都大饭店办公楼 405 单元

Phone: +86-21-62489820

Fax: $+86-21-62489821$ 
(C) 2011 The Author(s). Licensee IntechOpen. This chapter is distributed under the terms of the Creative Commons Attribution-NonCommercialShareAlike-3.0 License, which permits use, distribution and reproduction for non-commercial purposes, provided the original is properly cited and derivative works building on this content are distributed under the same license. 